\title{
Existence and uniqueness for a free boundary problem arising in combustion theory
}

\author{
F. HAMEL ${ }^{\dagger}$ \\ CNRS, Laboratoire d'Analyse Numérique, Université Pierre et Marie Curie 4, place Jussieu, \\ 75252 Paris Cedex 05, France \\ AND \\ R. MONNEAU \\ CERMICS-ENPC, 6-8 avenue B. Pascal, Cité Descartes, Champs-sur-Marne, \\ 77455 Marne-La-Vallée Cedex 2, France
}

[Received 30 January 2001 and in revised form 29 November 2001]

\begin{abstract}
This paper deals with the questions of the existence and uniqueness of solutions to a problem with a conical-shaped free boundary. It is also concerned with providing a complete classification of the solutions to a more abstract Serrin-type free boundary problem. These solutions are proved to be either conical-shaped or planar. Such problems arise in the modelling of premixed equidiffusional Bunsen flames in the limit of high activation energies.
\end{abstract}

Keywords: Free boundary problem; travelling fronts; conical shape; overdetermined elliptic equations; sliding method.

\section{Introduction and main results}

The aim of this paper is to find the solutions $(c, u, \Omega)$ of the following free boundary problem:

$$
\left\{\begin{aligned}
\Delta u-c \partial_{y} u & =0 \text { in } \Omega \subset \mathbb{R}^{2}, \\
u & =1 \text { in } \mathbb{R}^{2} \backslash \Omega, \\
0<u & <1 \text { in } \Omega, \\
\partial_{n} u & =c_{0}>0 \text { on } \Gamma=\partial \Omega, \\
u(X) & =0, \\
\limsup _{d(X, \Gamma) \rightarrow+\infty, X \in \Omega} &
\end{aligned}\right.
$$

where $X=(x, y)$ is the generic notation for the points of $\mathbb{R}^{2}, c \in \mathbb{R}, u$ is a globally Lipschitzcontinuous function and $\Omega$ is a an open set. Here, $n$ is the outward unit normal to the set $\Omega$ and $\partial_{n} u$ stands for the normal derivative on $\Gamma$ of the restriction of the function $u$ to the set $\bar{\Omega}$, in the case where $u$ is smooth enough in $\Omega$ up to the boundary. More precise statements on the regularity of $u$ will be made later.

In some statements, we will assume moreover that $\Omega$ is a smooth sub-graph

$$
\Omega=\{y<\phi(x)\}
$$

Present address: Univesité Aix-Marseille III, LATP, Faculté Saint-Jérôme, Avenue Escadrille Normandie-Niemen, 13397 Marseille Cedex 20, France. 

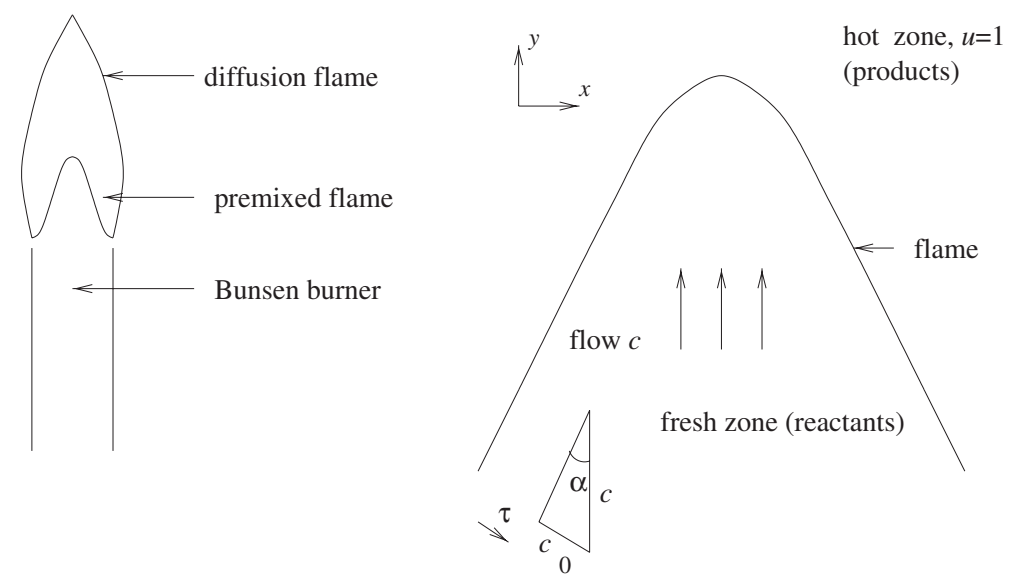

FIG. 1. Bunsen flames, and detail of the premixed flame.

$\phi$ being an unknown smooth function such that

$$
\phi^{\prime}(x) \rightarrow \mp \cot \alpha \text { as } x \rightarrow \pm \infty \text {. }
$$

The parameter $\alpha \in(0, \pi)$ is a given angle. The unknown function $u$ satisfies overdetermined conditions on $\Gamma$ but the boundary $\Gamma=\partial\{u<1\}$ is unknown as well. The unknown velocity $c$ can be seen as an eigenvalue and $u$ as an eigenfunction to the nonlinear eigenvalue problem (1.1).

We prove the existence of solutions $(c, u, \Omega)$ to (1.1)-(1.2). Under some more general assumptions on $\Omega$ and $\Gamma$, we classify all the solutions of (1.1) and we prove in particular that the solutions of (1.1)-(1.2) are unique.

Before stating our main results, we first explain the physical motivation of the problem we are interested in.

\subsection{Physical motivation}

Before going any further on the mathematical results, let us first describe the physical motivation of this problem, namely a simple model for laminar and steady Bunsen flames. At the mouth of a Bunsen burner, two flames can usually be seen: a conical-shaped premixed flame and, beyond, a diffusion flame, as shown in Fig. 1 (see Buckmaster and Ludford [16, 17], Joulin [30], Lewis and Von Elbe [35], Liñan [36], Sivashinsky [45, 46] or Williams [51]). We focus here on the study of the premixed flame. In the limit of high activation energy for the chemical reaction rate, the reaction is located on an infinitely thin zone, which is usually referred to as the flame front. The fresh mixture (fuel and oxidizer) is located below the flame front and, there, the temperature is not high enough for the reaction to ignite. Above the flame, there are only the burned gases and the reaction cannot take place either, because of the lack of one of the reactants.

We stand within the framework of the thermal-diffusional model, with constant density $([8,16,17,37,52])$. We consider a unit Lewis number, meaning that the coefficients of thermal conductivity and molecular diffusion are identical—which is usually a good approximation — and an overall one-step irreversible and exothermic chemical reaction. We do not investigate here the profile of the flame near the burner rim. The flame is assumed to be stabilized in a uniform and 
adiabatic stream coming from the burner. Following Williams ( [51: chapter 5]), we deal with a two-dimensional geometry, which is equivalent to considering a Bunsen burner with an elongated rectangular outlet.

Mathematically speaking, the flame can be viewed as a conical-shaped free boundary between two zones, a hot one and a relatively fresh one. In this paper, we will be concerned with the rigorous derivation of the shape of these flames under that Dirac approximation for the chemical reaction. Because of the invariance of the shape of the flame with respect to the size of the burner, we consider the problem in the whole space $\mathbb{R}^{2}=\{X=(x, y)\}$ calling $y$ the main direction of the stream and $x$ the direction orthogonal to $y$ (see Figure 1). Under all the above assumptions, the temperature field $u(X)$, adimensionalized and renormalized in such a way that $u \simeq 0$ far below the flame and $u=1$ on the flame and above it, solves the following equations:

$$
\left\{\begin{array}{c}
\Delta u-c \partial_{y} u=0 \text { in } \Omega=\{0<u<1\}, \\
u=1 \text { in } \mathbb{R}^{2} \backslash \Omega, \\
\limsup u(X)=0, \\
d(X, \Gamma) \rightarrow+\infty, X \in \Omega \\
u \text { is continuous across } \Gamma:=\partial \Omega,
\end{array}\right.
$$

where the curve $\Gamma$, which we write as $\Gamma=\{y=\phi(x)\}$, represents the flame front, $\Omega$ is the 'fresh' zone and $c$ is the normalized velocity of the underlying upward stream. Here, $d(X, \Gamma)$ is the distance between the point $X=(x, y)$ and the curve $\Gamma$. In this model, the function $1-u$ represents the relative concentration of one of the reactants. The continuity of $u$ across $\Gamma$ means that there is no jump of the temperature on the flame front. Notice that despite its simplicity, this model retains the fundamental features for the description of the premixed Bunsen flames: first, in the zone below the flame, heat conduction and convection by the flow are taken into account; then, as we shall see later, on the interface $\Gamma$ the chemical reaction (fuel/oxidizer consumption) is accounted for under the form of an additional condition for $u$ across $\Gamma$.

In experiments $([16,35,51])$, the flame front is seen to be almost planar far away from the tip and to have two asymptotic directions making an angle $\alpha(0<\alpha \leqslant \pi / 2)$ with respect to the direction $-e_{y}=(0,-1)$, as shown in Fig. 1). In other words, it is reasonable to say that $\phi$ satisfies (1.2).

In the one-dimensional case, the free interface $\Gamma$ reduces to a single point, say $\{0\}$, and the problem reads

$$
\left\{\begin{array}{r}
U_{0}^{\prime \prime}-c_{0} U_{0}^{\prime}=0 \text { in }\{y<0\}, \\
U_{0}=1 \text { in }\{y \geqslant 0\}, \\
U_{0}(y) \rightarrow 0 \text { as } y \rightarrow-\infty, \\
U_{0} \text { is continuous at the point } 0 .
\end{array}\right.
$$

The function $U_{0}(y)$ is then equal to $U_{0}(y)=\mathrm{e}^{c_{0} y}$ if $y \leqslant 0$ and $U_{0}(y)=1$ if $y \geqslant 0$. The gradient of $U_{0}$ necessarily satisfies the following jump condition at 0 :

$$
\left(U_{0}\right)_{-}^{\prime}(0):=\lim _{\substack{y \rightarrow 0 \\<}} \frac{U_{0}(y)-U_{0}(0)}{y}=c_{0} .
$$

Such planar solutions exist for each given positive number $c_{0}$. In practice, this real number only depends on some physical characteristics of the problem and it is referred to as the planar burning velocity. In what follows, $c_{0}$ is a given and arbitrary positive real number. 
Coming back to the multidimensional case and in regard of the one-dimensional analysis, the following jump condition for the gradient of $u$ is required at any point of the free interface $\Gamma=$ $\{y=\phi(x)\}$ :

$$
\frac{\partial u}{\partial n}=c_{0} \text { on } \Gamma,
$$

where $n$ is the unit normal to $\Gamma$ pointing outside the zone $\Omega=\{u<1\}$ (we assume for the time being that the curve $\Gamma$ is smooth), and where $\frac{\partial u}{\partial n}$ stands for the normal derivative on $\Gamma$ of the restriction of $u$ to the set $\bar{\Omega}$. This jump condition means that the normal heat production and the burning velocity are constant over the wave surface (for the derivation of (1.5), see also [15] [17]). Formula (1.5) could also be obtained by analogy with (1.4) and by formally thinking of the interface as almost flat and the flame as almost planar in the vicinity of any point on $\Gamma$. Note that interface conditions of the type (1.5) have been used in many such combustion problems, e.g. $[2,12,14,19,20,23,29,34]$.

It is now natural to wonder what could be the relation between the planar flame speed $c_{0}$ and the vertical stream velocity $c$. Let us think of the flame front as a conical-shaped reaction wave spreading at the vertical speed $c$ through a quiescent gaseous premixture. Far away from the tip, say on the left as $x \rightarrow-\infty$, the flame is almost planar and it should then move at the speed $c_{0}$ in the direction $\tau=(\cos \alpha,-\sin \alpha)$, which is asymptotically normal to the flame surface as drawn in Fig. 1. Hence, $c_{0}$ is the projection of the speed $c$ over the vector $\tau$. That means that

$$
c=\frac{c_{0}}{\sin \alpha} .
$$

This formula, which can also be found in [16], [35] or [46], in particular implies that the larger the intensity of the flow at the exit of the burner is, the sharper the flame is. In experiments, the planar burning velocity $c_{0}$ can be determined from the knowledge of the flow velocity $c$ and from the measurement of the apex angle $2 \alpha$ of the flame cone (see e.g. Williams [51]).

\subsection{A brief overview of related results in the literature}

The question of finding solutions to the free boundary problem (1.1), given the formula (1.6), was first investigated by Buckmaster and Ludford [15-17] in the modelling of near-equidiffusional flame tips (they actually considered a system with two unknown functions). They solved the problem in the formal limit of large flow intensity ( $c \rightarrow+\infty$, i.e. $\alpha \rightarrow 0)$ and they especially showed that, as $c \rightarrow+\infty$, the tip might then break open for Lewis numbers smaller enough than 1 . The limit $\alpha \rightarrow 0$ corresponds to slender flames, a situation very different from the one we consider here. Mathematically speaking, the original elliptic free boundary problem is formally replaced with a parabolic problem in terms of the variables $x$ and $y^{\prime}=y / c$.

Other formal works had been devoted to the analysis of the premixed Bunsen flames viewed as thin interfaces. From multiple-scale asymptotic expansions, Sivashinsky [46] derived a firstorder equation for the flame front in terms of the new variable $x^{\prime}=x / E$ in the limit $E \rightarrow+\infty$. Sivashinsky showed that the flame front was smooth if the Lewis number was strictly greater than 1 and that, in contrast, the tip was wedge-shaped if the Lewis number was strictly less than 1 (for further results, including the three-dimensional case, see $[33,35,45,46])$.

Another approach was used by Michelson [38]. In the case of a unit Lewis number, he considered the flame front $\left\{y=\phi^{\alpha}(x)\right\}$ as a curve satisfying the slope condition (1.2) at infinity and solving the 
fourth-order Kuramoto-Sivashinsky equation-an approach which is different from the one used in this paper (see [13], [22], [47] or [48] for a derivation of this Kuramoto-Sivashinsky equation). Michelson proved the existence, and also the uniqueness, of such solutions $\phi^{\alpha}$ as soon as the angle $\alpha$ of the flame is not too far from 0 (for three-dimensional results, see [39]). He also pointed out that the uniqueness of the profile of the front was not expected for angles $\alpha$ close to $\pi / 2$.

Conical fronts also arise in different frameworks. For instance, they can be used to describe the propagation of curved interfaces in mean curvature flows with constant driving force. Ninomiya and Taniguchi $[41,42]$ have studied the existence and the dynamical aspects of such interfaces.

\subsection{Main results of the paper}

Our goal in this paper is to make a rigorous analysis of the conical shape of the premixed Bunsen flame for the model described in Section 1.1. Namely, we want to prove the existence of a function $u$, a (free) domain $\Omega$ with (free) boundary $\Gamma$ and a speed $c$ satisfying (1.1), the angle $\alpha$ and the speed $c_{0}$ being given. We also derive rigorously the formula (1.6) for the speed $c$. In this paper, we moreover answer the questions of the uniqueness and other qualitative properties of the solutions (monotonicity, smoothness, asymptotic behaviour of the free boundary). Lastly, we classify all solutions of a related Serrin-type problem.

Let us first state the existence of a solution $\left(c^{\alpha}, u^{\alpha}, \Omega^{\alpha}\right)$ to (1.1)-(1.2) for each angle $0<\alpha \leqslant$ $\pi / 2$.

Theorem 1.1 For each $\alpha \in\left(0, \frac{\pi}{2}\right]$, there exists a solution $\left(c^{\alpha}, u^{\alpha}, \Omega^{\alpha}\right)$ to the free boundary problem (1.1)-(1.2) satisfying

$$
c^{\alpha}=\frac{c_{0}}{\sin \alpha} .
$$

The function $u^{\alpha}$ is globally Lipchitz-continuous, it is symmetric with respect to the axis $\{x=0\}$ and it is nonincreasing in each direction of the 'lower' cone

$$
\mathcal{C}^{-}(\alpha)=\left\{\lambda \tau, \lambda \geqslant 0,\|\tau\|=1, \tau_{y} \leqslant-\cos \alpha\right\} .
$$

The set $\Omega^{\alpha}$ is of the type

$$
\Omega^{\alpha}=\left\{y<\phi^{\alpha}(x)\right\}
$$

and the function $\phi^{\alpha}$ is even, globally Lipschitz-continuous and it satisfies

$$
\frac{\mathrm{d} \phi^{\alpha}}{\mathrm{d} x}(x) \leqslant 0 \text { for all } x \geqslant 0 .
$$

Moreover the free boundary $\Gamma^{\alpha}=\partial \Omega^{\alpha}$ is analytic with globally bounded curvature, the restriction of $u^{\alpha}$ to $\overline{\Omega^{\alpha}}$ is analytic up to $\Gamma^{\alpha}$ and $\Gamma^{\alpha}$ has two asymptots parallel to the half-lines $y=-|x| \cot \alpha$, namely

$$
\exists L \in \mathbb{R}, \quad \phi^{\alpha}(x)+|x| \cot \alpha \rightarrow L \text { as } x \rightarrow \pm \infty .
$$

Remark 1.2 This result enables us, in particular, to find a solution of the flame tip problem of Buckmaster and Ludford [17], just by taking the function $u^{\alpha}$ restricted on the half-plane $\{x \geqslant 0\}$.

Let us now turn to the question of the uniqueness of the solutions of the free boundary problem (1.1)-(1.2). The following theorem deals with some uniqueness properties and it also answers the question of the nonexistence of solutions with angles $\alpha>\pi / 2$. 
Theorem 1.3 Let $c_{0}>0$ and $0<\alpha<\pi$ be given. Consider a solution $(c, u, \Omega)$ to the free boundary problem (1.1)-(1.2), where the restriction of $u$ to $\bar{\Omega}$ is $C^{1}$ on $\bar{\Omega}$ and $\Omega$ is of the type $\Omega=\{y<\phi(x)\}$. Assume that $\Gamma=\partial \Omega$ is globally $C^{1,1}$ with bounded curvature, and that $\phi$ is globally Lipschitz.

Then $\alpha \leqslant \pi / 2$ and $(c, u, \Omega)$ is unique in the sense that

$$
c=\frac{c_{0}}{\sin \alpha}
$$

and there exists a vector $(a, b) \in \mathbb{R}^{2}$ such that $u(x, y)=u^{\alpha}(x+a, y+b)$ for all $(x, y) \in \mathbb{R}^{2}$ and $\Omega=\left\{(x, y),(x+a, y+b) \in \Omega^{\alpha}\right\}$.

It follows from Theorems 1.1 and 1.3 that the free boundary problem (1.1)-(1.2) is well-posed for any angle $\alpha \in(0, \pi / 2]$ whereas no solution exists whenever $\alpha$ is larger than $\pi / 2$ (i.e. $c$ smaller than $c_{0}$ ). Note that despite its simplicity the model we have used is robust enough to capture that the tip of the flame cannot point downwards, as has been observed in experiments.

Remark 1.4 In the particular case $\alpha=\pi / 2$, any solution $u$ of (1.1)-(1.2) has one-dimensional symmetry: namely, it depends on $y$ only, and $\Omega$ is a half-space of the type $\{y<h\}$.

Remark 1.5 The method we use to prove this theorem allows for additional a priori estimates in $\mathbb{R}^{N}=\left\{(x, y), x \in \mathbb{R}^{N-1}, y \in \mathbb{R}\right\}$ for dimensions $N \geqslant 3$. Nevertheless, the question of the existence of solutions with $\alpha<\pi / 2$ is still open in dimensions 3 or higher.

Let us now observe that the free boundary problem (1.1), without assuming any slope condition at infinity like (1.2) for the free boundary $\Gamma$, can also be viewed as a Serrin-type problem. Indeed, the unknown function $u$ satisfies the elliptic equation

$$
\Delta u-c \partial_{y} u=0
$$

in the unknown set $\Omega=\{u<1\}$, and overdetermined conditions are required on the boundary $\Gamma=\partial \Omega$ :

$$
u=1 \text { and } \partial_{n} u=c_{0}>0
$$

as are uniform limiting conditions $\lim \sup _{d(X, \Gamma) \rightarrow+\infty, X \in \Omega} u(X)=0$ far away from the free boundary $\Gamma$.

Problems of this kind were investigated by Serrin [44]. Serrin proved that, if $u$ is a positive solution of an equation of the type $\Delta u+f(u)=0$ in a smooth-bounded domain $\Omega$, given overdetermined boundary conditions: $u=0$ and $\partial_{n} u=a$ on $\partial \Omega$, then, under some assumptions on the function $f, \Omega$ is a ball and the function $u$ is radially symmetric with respect to the centre of the ball (see also Henrot and Philippin [28] for similar results on related eigenvalue problems). The same result as Serrin has been extended by Reichel [43] and Aftalion and Busca [1] for exterior domains, under various assumptions on the nonlinearity $f$. Similar free boundary problems in Lipschitz domains of the type $\Omega=\left\{x_{n}<\phi\left(x^{\prime}\right), x^{\prime}=\left(x_{1}, \ldots, x_{N-1}\right) \in \mathbb{R}^{N-1}\right\}$ have been investigated by Berestycki et al. [5]: under some conditions on the nonlinearity $f$ and under a flatness condition on the function $\phi$ at infinity $\left(\forall \tau \in \mathbb{R}^{N-1}, \lim _{\left|x^{\prime}\right| \rightarrow+\infty} \phi\left(x^{\prime}+\tau\right)-\phi\left(x^{\prime}\right)=0\right)$, then $\Omega$ is a half-space, i.e. $\phi$ is constant, and the solution $u$ only depends on $x_{n}$.

Let us now try to classify all the solutions $(u, c, \Omega)$ of the free boundary problem (1.1), dropping the slope condition (1.2) for $\Gamma$. Because of the term $c \partial_{y} u$, one cannot expect any radial symmetry 
property here. Under some smoothness assumptions for $\Gamma$, we shall actually prove that, besides some trivial planar solutions, the solutions given in Theorem 1.1 are the only solutions of (1.1). To be more precise, we show in this paper the following theorem, which Theorem 1.3 is actually a consequence of

Theorem 1.6 Let $(c, u, \Omega)$ be a solution of the free boundary problem (1.1), where $\Omega$ is an open set such that both $\Omega$ and $\mathbb{R}^{2} \backslash \Omega$ are not empty, and the restriction of $u$ to $\bar{\Omega}$ is $C^{1}$ in $\bar{\Omega}$. Assume that the free boundary $\Gamma=\partial \Omega$ is globally $C^{1,1}$ with a bounded curvature. Assume moreover that $\mathbb{R}^{2} \backslash \Omega$ has no bounded connected components.

Then, even if it means changing $(c, u, \Omega)$ into $(-c, u(-x,-y),-\Omega)$, one has $c \geqslant c_{0}$ and, if $\alpha \in(0, \pi / 2]$ denotes the only solution of $c=c_{0} / \sin \alpha$, the following three and only three cases (up to translation) may occur:

- either $\Omega$ is the half-space $\{y<x \cot \alpha\}$ and

$$
u(x, y)=U_{0}(y \sin \alpha-x \cos \alpha)
$$

where $U_{0}$ solves the one-dimensional free boundary problem (1.3)-(1.4),

- or the same conclusion holds up to symmetry in $x: \Omega=\{y<-x \cot \alpha\}$ and $u(x, y)=$ $U_{0}(y \sin \alpha+x \cos \alpha)$,

- or $\Omega=\Omega^{\alpha}$ and $u=u^{\alpha}$ where $\left(u^{\alpha}, \Omega^{\alpha}\right)$ is the solution of (1.1)-(1.2) given in Theorem 1.1.

Remark 1.7 In the particular case $c=c_{0}$, then, under the regularity and connectedness assumptions on $\Omega$, any solution $(u, \Omega)$ of (1.1) is planar: $\Omega$ is a half-space of the type $\Omega=$ $\{ \pm(y-h)<0\}$ for some $h \in \mathbb{R}$ and $u$ only depends on the variable $y$, namely, $u(x, y)=\mathrm{e}^{ \pm c_{0}(y-h)}$.

Remark 1.8 The solutions $u$ in Theorem 1.6 eventually turn out to be much smoother than the assumption of $C^{1}$ regularity up to the boundary, which is enough in the proof. The same theorem would be true if classical $C^{2}$ regularity up to the boundary was assumed. On the other hand, the assumption of the boundedness of the curvature of the free boundary plays a crucial role: together with classical a priori estimates for the function $u$, it guarantees local uniform properties of the boundary (Proposition 3.7 below). As a consequence, compactness properties hold (Proposition 4.2 below). These compactness properties hold for the solutions of a more general class of free boundary problems, but, in order to avoid many technical definitions in the introduction, this more general class of free boundary problems is defined in Section 3.

Let us, however, notice that the class of free boundary problems defined in Section 3 is stronger than the definitions in the sense of Caffarelli. A uniformity property of the behaviour of the functions $u$ in a neighbourhood of the boundary, which is satisfied from the assumptions of Theorem 1.6 (see Proposition 3.7), is indeed used in our proofs and especially in some compactness results.

Closely related to the above theorem are the following open question and conjecture.

Open question. Is Theorem 1.6 true if it is only assumed that $|c| \geqslant c_{0}$, without assuming that $\mathbb{R}^{2} \backslash \Omega$ has no bounded connected components?

Conjecture. In dimension $N \geqslant 2$, there exist non-trivial solutions with $\mathbb{R}^{N} \backslash \Omega$ bounded.

The main difficulties in dealing with the above free boundary problems lie in the fact that we are here concerned with a multidimensional problem in an unbounded domain - the whole plane $\mathbb{R}^{2}$ and that little information on the behaviour at infinity of the function $u$ and of the free interface $\Gamma$ are 
available. Furthermore, because of the first-order term $\partial_{y} u$, the problem is not put in the divergence form and there is no natural variational formulation.

The strategy we adopt in Section 2 to prove the existence of solutions of (1.1) together with the limiting conditions (1.2) consists in working with elliptic reaction-diffusion equations in the whole plane $\mathbb{R}^{2}$ which are regularizing approximations of the free boundary problem (1.1). The existence of solutions of such reaction-diffusion equations with conical conditions at infinity has been obtained by Bonnet and Hamel [11]. Then, a solution of the free boundary problem (1.1) is obtained as a singular limit of the solutions of these reaction-diffusion equations, following the results of Berestycki et al. [4] on similar problems in straight infinite cylinders. The key point in our framework is to show that the conical limiting conditions (1.2) carry over after passing to the limit with respect to the regularization parameter.

The Serrin-type result (Theorem 1.6, including the uniqueness result of Theorem 1.3) is proved in Section 5. To prove this, we state in Sections 3 and 4 some monotonicity properties and a general comparison principle for the solutions of a class of free boundary problems. The proof of this comparison principle is given in Section 6 and is based on the sliding method developed by Berestycki and Nirenberg [10].

\section{Existence of a solution of the free boundary problem (1.1)-(1.2): proof of Theorem 1.1}

This section is devoted to the proof of Theorem 1.1. It is divided into two main steps. The first step consists in stating some results of Bonnet and Hamel [11] about regularizing approximations of the free boundary problem (1.1)-(1.2). The second step is concerned with proving the existence of a solution to the free boundary problem by passing to the limit as the regularization parameter approaches 0 .

Step 1: regularizing approximations. Let $\alpha$ be a given angle $\alpha \in(0, \pi / 2]$ and let $c_{0}>0$ be given. We are now going to replace the underlying $\delta$-approximation for the reaction rate by a sequence of nonlinear source terms approximating a Dirac mass at the point 1.

In order to do so, let $f$ be a given function defined on $[0,1]$ and satisfying

$$
\left\{\begin{array}{c}
f \text { is Lipschitz-continuous on }[0,1] \\
\exists \theta \in(0,1), f \equiv 0 \text { on }[0, \theta], f>0 \text { on }(\theta, 1), f(1)=0, f^{\prime}(1)<0
\end{array}\right.
$$

together with

$$
c_{0}=\sqrt{2 \int_{0}^{1} f(s) \mathrm{d} s} .
$$

The real $\theta$ is then referred to as the ignition temperature for the nonlinear source term $f$. Without any loss of generality, we assume that $f$ is extended by 0 outside the interval $[0,1]$. Now let $\left(f_{\varepsilon}\right)_{\varepsilon>0}$ be the sequence of functions defined by

$$
\forall s \in[0,1], \quad f_{\varepsilon}(s)=\frac{1}{\varepsilon} f\left(1-\frac{1-s}{\varepsilon}\right) .
$$

The choice of the functions $f_{\varepsilon}$ can be derived from Arrhenius kinetics. These functions $f_{\varepsilon}$ approximate the Dirac distribution at $s=1$ with the mass $\int_{0}^{1} f$. Note that the limit $\varepsilon \rightarrow 0$ is of physical interest since the quantity $1 / \varepsilon$ is a normalized activation energy. At the limit, the chemical reaction cannot ignite below the temperature $u=1$. 
Before going any further, let us state the following results of Bonnet and the first author about the regularized counterpart of the free boundary problem (1.1)-(1.2).

Theorem 2.1 (Bonnet and Hamel [11]) Let $f$ be a function satisfying (2.1). For each $\alpha \in(0, \pi / 2]$, there exists a solution $\left(c_{1}^{\alpha}, u_{1}^{\alpha}\right)$ of the following problem:

$$
\left\{\begin{array}{r}
\Delta u_{1}^{\alpha}-c_{1}^{\alpha} \partial_{y} u_{1}^{\alpha}+f\left(u_{1}^{\alpha}\right)=0 \text { in } \mathbb{R}^{2}, \\
\forall \tau \in\left\{\|\tau\|=1, \tau_{y}<-\cos \alpha\right\}, \quad \lim _{\lambda \rightarrow+\infty} u_{1}^{\alpha}(\lambda \tau)=0, \\
\forall \tau \in\left\{\|\tau\|=1, \tau_{y}>-\cos \alpha\right\}, \lim _{\lambda \rightarrow+\infty} u_{1}^{\alpha}(\lambda \tau)=1, \\
\forall \tau \in\left\{\|\tau\|=1, \tau_{y} \leqslant-\cos \alpha\right\}, \quad \partial_{\tau} u_{1}^{\alpha} \leqslant 0, \\
u_{1}^{\alpha}(x, y)=u_{1}^{\alpha}(-x, y) \text { in } \mathbb{R}^{2}, \\
\partial_{x} u_{1}^{\alpha} \geqslant 0 \text { in }\{x \geqslant 0\}
\end{array}\right.
$$

such that $u_{1}^{\alpha}(0,0)=\theta$. The speed $c_{1}^{\alpha}$ is unique and is given by

$$
c_{1}^{\alpha}=\frac{c_{1}}{\sin \alpha}>0,
$$

where $c_{1}$ is the unique speed for which there exists a one-dimensional solution $U_{1}$ of

$$
\left\{\begin{array}{l}
U_{1}^{\prime \prime}-c_{1} U_{1}^{\prime}+f\left(U_{1}\right)=0 \text { in } \mathbb{R} \\
U_{1}(-\infty)=0<U_{1}<U_{1}(+\infty)=1 .
\end{array}\right.
$$

Furthermore, there exists a positive constant $C$-for instance, $C=3$ works-such that, if $X_{0}=$ $\left(x_{0}, y_{0}\right)$ satisfies $u_{1}^{\alpha}\left(X_{0}\right) \leqslant \theta$, then

$$
u^{\alpha}\left(x_{0}, y\right) \leqslant C \mathrm{e}^{\left(y-y_{0}\right) c_{1} \sin \alpha} \text { for all } y \leqslant y_{0} .
$$

Remark 2.2 The existence of one-dimensional travelling fronts solving (2.5) is well known ([3], [9], [21] or [31]). Furthermore, the speed $c_{1}$ of such fronts is unique, and the front $U_{1}$ itself is unique up to translation.

Remark 2.3 Results stronger than those in Theorem 2.1 have actually been proved in [11]. In particular, the following inequality holds:

$$
u_{1}^{\alpha}(x, y) \leqslant 2 \theta \mathrm{e}^{-c_{1}\left|x_{0}\right| \cos \alpha} \cosh \left(c_{1} x \cos \alpha\right) \mathrm{e}^{c_{1}\left(y-y_{0}\right) \sin \alpha}+\theta \mathrm{e}^{c_{1}\left(y-y_{0}\right) / \sin \alpha}
$$

for all $(x, y) \in\left\{(x, y) \in \mathbb{R}^{2}, y \leqslant y_{0}, y \leqslant y_{0}+\left(x-x_{0}\right) \cot \alpha, y \leqslant y_{0}-\left(x+x_{0}\right) \cot \alpha\right\}$, whenever $x_{0} \leqslant 0$ and $u_{1}^{\alpha}\left(x_{0}, y_{0}\right) \leqslant \theta$. It is easy to check that the latter yields (2.6) with $C=3$. Additional results have also been obtained in [26] and [27].

For each $\varepsilon>0$ small enough, the function $f_{\varepsilon}$ defined in (2.3) satisfies (2.1) with the ignition temperature $\theta_{\varepsilon}=1-\varepsilon(1-\theta) \in(0,1)$. We can then apply Theorem 2.1 to it: there exists a solution $\left(c_{\varepsilon}^{\alpha}, u_{\varepsilon}^{\alpha}\right)$ of $(2.4)$ such that $u_{\varepsilon}^{\alpha}(0,0)=\theta_{\varepsilon}$. The speed $c_{\varepsilon}^{\alpha}$ is given by $c_{\varepsilon}^{\alpha}=c_{\varepsilon} / \sin \alpha$ where $c_{\varepsilon}$ is the unique speed for which problem (2.5) with $f=f_{\varepsilon}$ has a solution.

Step 2: passage to the limit $\varepsilon \rightarrow 0$. This step is devoted to proving that the solutions $\left(c_{\varepsilon}^{\alpha}, u_{\varepsilon}^{\alpha}\right)$ of the regularizing approximations (2.4), defined in the whole plane $\mathbb{R}^{2}$, converge as $\varepsilon \rightarrow 0$ to a solution of (1.1)-(1.2). One first uses some general convergence results of Berestycki et al. [4] for 
similar problems in straight infinite cylinders and one then proves that the function obtained at the limit is singular on a free boundary and has conical-shaped level curves, as the solutions of (2.4).

Consider a sequence $\varepsilon_{n} \rightarrow 0^{+}$. From a result of Berestycki et al. [9] (see also the pioneering paper of Zeldovich and Frank-Kamenetskii [53]), it is known that

$$
c_{\varepsilon_{n}} \rightarrow \sqrt{2 \int_{0}^{1} f}=c_{0} \quad \text { as } \varepsilon_{n} \rightarrow 0^{+},
$$

whence

$$
c_{\varepsilon_{n}}^{\alpha} \rightarrow c^{\alpha}:=\frac{c_{0}}{\sin \alpha} .
$$

The convergence of the functions $u_{\varepsilon_{n}}^{\alpha}$ uses some results of Berestycki et al. [4] summarized in the following theorem.

Theorem 2.4 (Berestycki et al. [4]) Let $B_{R}=B_{R}(X)$ be an open ball of radius $R$ and centre $X \in \mathbb{R}^{2}$. Let $f$ satisfy (2.1)-(2.2) and let $f_{\varepsilon}$ be the functions defined by (2.3). Let $\left(c_{\varepsilon}\right)_{\varepsilon}$ be a sequence such that $c_{\varepsilon} \rightarrow c_{0}$ as $\varepsilon \rightarrow 0$. Let $\left(u_{\varepsilon}\right)_{\varepsilon}$ be a sequence of functions satisfying

$$
\Delta u_{\varepsilon}-c_{\varepsilon} \partial_{y} u_{\varepsilon}+f_{\varepsilon}\left(u_{\varepsilon}\right)=0 \text { in } B_{R} .
$$

Then, in $B_{R / 2}=B_{R / 2}(X)$, the functions $u_{\varepsilon}$ are uniformly Lipschitz-continuous with Lipschitz constant depending only on $\sup _{\varepsilon} c_{\varepsilon}, \max _{[0,1]} f$ and $R$.

Assume now that $u$ is a function such that $u_{\varepsilon} \rightarrow u$ uniformly in $B_{R / 2}$. Let $\Gamma=\partial\{u<1\} \cap B_{R / 2}$. Then, on regular parts of $\Gamma$,

$$
\partial_{n} u=c_{0},
$$

where $n$ is the exterior normal to the set $\{u<1\}$ on $\Gamma$.

Remark 2.5 Similar one-dimensional results had already been obtained for systems of two equations in [9] and for more general functions $f_{\varepsilon}$ in [25]. Furthermore, results more general than Theorem 2.4 on regularizing approximations of free boundary problems with nonuniform velocity fields can be found in [4] (see also [19, 20,34] for other results in the elliptic or parabolic cases).

As for (1.5), the condition (2.7) in Theorem 2.4 says that the gradient of the function obtained at the limit has a constant jump on the free boundary $\Gamma$. This condition can then also be viewed as a memory of the strongly temperature-dependent reaction rate.

One can now move the centre of $B_{R}$ everywhere in $\mathbb{R}^{2}$ and it follows from the above theorem that the functions $u_{\varepsilon_{n}}^{\alpha}$ are globally Lipschitz in $\mathbb{R}^{2}$ with Lipschitz norms that do not depend on $\varepsilon_{n}$. Up to extraction of a subsequence, they converge to a globally Lipschitz function $u^{\alpha}$ uniformly on the compact subsets of $\mathbb{R}^{2}$. The function $u^{\alpha}$ satisfies $0 \leqslant u^{\alpha} \leqslant 1$ in $\mathbb{R}^{2}$. Let

$$
\Omega^{\alpha}=\left\{u^{\alpha}<1\right\} \text { and } \Gamma^{\alpha}=\partial \Omega^{\alpha} .
$$

We now aim at proving that $\left(c^{\alpha}, u^{\alpha}, \Omega^{\alpha}\right)$ is a solution of (1.1)-(1.2). Let us first observe that $f_{\varepsilon_{n}}=0$ on $\left[0, \theta_{\varepsilon_{n}}\right]$ where $\theta_{\varepsilon_{n}}=1-\varepsilon_{n}(1-\theta) \rightarrow 1$ as $\varepsilon_{n} \rightarrow 0$. Therefore, by passage to the limit $\varepsilon_{n} \rightarrow 0^{+}$, the function $u^{\alpha}$ satisfies

$$
\Delta u^{\alpha}-c^{\alpha} \partial_{y} u^{\alpha}=0 \text { in } \Omega^{\alpha}
$$


and $u^{\alpha}(0,0)=1$. Similarly, the inequality (2.6) applied to $u_{\varepsilon_{n}}^{\alpha}$ implies that

$$
u^{\alpha}(0, y) \leqslant C \mathrm{e}^{y c_{0} \sin \alpha} \text { for all } y \leqslant 0 .
$$

Up to translation in the direction $y$, one can assume that $u(0, y)<1$ for all $y<0$. It follows in particular that $\Omega^{\alpha} \neq \emptyset$. Notice also that $u^{\alpha}>0$ in $\Omega^{\alpha}$ from the strong maximum principle.

From Theorem 2.1, $u^{\alpha}$ is symmetric with respect to $x$ and nondecreasing in $x$ in the half-space $\{x \geqslant 0\}$. Hence, $u^{\alpha}(x, 0)=1$ for all $x \in \mathbb{R}$. Furthermore, the function $u^{\alpha}$ is nonincreasing in any direction $\tau$ such that $\|\tau\|=1$ and $\tau_{y} \leqslant-\cos \alpha$. Together with (2.8), that yields

$$
\lim _{b \rightarrow-\infty} \sup _{\mathcal{C}^{-}(b, \alpha)} u^{\alpha}=0,
$$

where $\mathcal{C}^{-}(b, \alpha)=\left\{(0, b)+\lambda \tau, \lambda \geqslant 0,\|\tau\|=1, \tau_{y} \leqslant-\cos \alpha\right\}$. Moreover, one can define the function $\phi^{\alpha}$ as follows:

$$
\forall x \in \mathbb{R}, \quad \phi^{\alpha}(x)=\inf \left\{y, u^{\alpha}(x, y)=1\right\} .
$$

Since $u^{\alpha}$ is nondecreasing in $y$, it follows that

$$
\Omega^{\alpha}=\left\{y<\phi^{\alpha}(x)\right\} .
$$

Proof of $\lim \sup u^{\alpha}(X)=0$ as $d\left(X, \Gamma^{\alpha}\right) \rightarrow+\infty, X \in \Omega^{\alpha}$. The function $u^{\alpha}$ being symmetric in $x$, the function $\phi^{\alpha}$ is even. Furthermore, $u^{\alpha}$ is nondecreasing with respect to $x$ in $\{x \geqslant 0\}$. As a consequence, $x \mapsto \phi^{\alpha}(x)$ is nonincreasing for $x \geqslant 0$. Similarly, since $u^{\alpha}$ is nonincreasing in both directions $( \pm \sin \alpha,-\cos \alpha)$, it follows that $\phi^{\alpha}$ is Lipschitz and that $\operatorname{Lip}\left(\phi^{\alpha}\right) \leqslant \cot \alpha$. We now claim that

$$
u^{\alpha}(x, y) \leqslant C \mathrm{e}^{\left(y-\phi^{\alpha}(x)\right) c_{0} \sin \alpha} \text { for all }(x, y) \in \overline{\Omega^{\alpha}} .
$$

Indeed, let $x \in \mathbb{R}$ be given and choose any $y_{0}<\phi^{\alpha}(x)$. Since $u_{\varepsilon_{n}}^{\alpha}\left(x, y_{0}\right) \rightarrow u^{\alpha}\left(x, y_{0}\right)<1$ as $\varepsilon_{n} \rightarrow 0$, one has $u_{\varepsilon_{n}}^{\alpha}\left(x, y_{0}\right) \leqslant \theta_{\varepsilon_{n}}$ for $\varepsilon_{n}$ small enough, whence

$$
u_{\varepsilon_{n}}^{\alpha}(x, y) \leqslant C \mathrm{e}^{\left(y-y_{0}\right) c_{\varepsilon_{n}} \sin \alpha} \text { for all } y \leqslant y_{0} .
$$

Passing to the limit $\varepsilon_{n} \rightarrow 0$ and $y_{0} \rightarrow \phi^{\alpha}(x)$ leads to (2.10).

Since $\phi^{\alpha}$ is globally Lipschitz, (2.10) yields that

$$
\limsup _{d\left(X, \Gamma^{\alpha}\right) \rightarrow+\infty, X \in \Omega^{\alpha}} u^{\alpha}(X)=0 .
$$

Regularity properties. From recent results of Berestycki et al. [6], generalizing some results of Caffarelli [18], the Lipschitz free boundary $\Gamma^{\alpha}=\left\{y=\phi^{\alpha}(x)\right\}$ is then globally $C^{1, \beta}$ for every $0<\beta<1$. The restriction of the function $u^{\alpha}$ to the set $\overline{\Omega^{\alpha}}$ is then of class $C^{1}$ up to the boundary $\Gamma^{\alpha}$. From Theorem 2.4, the smoothness of $\Gamma^{\alpha}$ implies that

$$
\partial_{n} u^{\alpha}=c_{0} \text { everywhere on } \Gamma^{\alpha},
$$

where $n$ is the exterior normal to $\Omega^{\alpha}$ on $\Gamma^{\alpha}$. From the general regularity theory of Kinderlehrer $e t$ al. [32], the free boundary $\Gamma^{\alpha}$ is of class $C^{2}$ and even analytic, and the restriction of the function $u^{\alpha}$ to the set $\overline{\Omega^{\alpha}}$ is analytic up to the boundary $\Gamma^{\alpha}$. Moreover, the following theorem, which directly follows from the methods developed by Kinderlehrer et al., holds. 
Theorem 2.6 (Kinderlehrer et al. [32]) Under the previous assumptions, and since $\Gamma^{\alpha}=$ $\left\{y=\phi^{\alpha}(x)\right\}$ is smooth, for every $a \in \mathbb{R}$ and $L>0$, there exists a constant $C=$ $C\left(c, c_{0}, L, \operatorname{Lip}\left(\mathrm{u}^{\alpha}\right), \operatorname{Lip}\left(\phi^{\alpha}\right)\right)>0$ such that

$$
\left|\left(\phi^{\alpha}\right)^{\prime \prime}\right|_{L^{\infty}(a-L / 2, a+L / 2)} \leqslant C .
$$

In particular, because the constant in (2.11) does not depend on the parameter $a$, we deduce that $\left(\phi^{\alpha}\right)^{\prime \prime}$ is globally bounded, i.e. $\Gamma^{\alpha}$ has a bounded curvature.

The boundary $\Gamma^{\alpha}$ has two asymptotes as $x \rightarrow \pm \infty$. We shall use here a few auxiliary lemmas. For the sake of simplicity, we drop the index $\alpha$ in the rest of this section. In particular, $\phi$ denotes the function $\phi^{\alpha}$.

Lemma 2.7 The function $v(x)=\int_{-\infty}^{\phi(x)} u(x, y) \mathrm{d} y$ is of class $C^{\infty}(\mathbb{R})$. Moreover, it satisfies

$$
v^{\prime \prime}(x)=c+\phi^{\prime \prime}(x)-c_{0} \sqrt{1+\phi^{\prime 2}(x)}
$$

and there exists a constant $C>0$ such that $\left\|v^{\prime}\right\|_{\infty} \leqslant C$.

Proof. In this lemma, the partial derivatives of $u$ stand for the partial derivatives of the restriction of the function $u$ to $\bar{\Omega}$.

From the inequality (2.10), namely

$$
\forall(x, y) \in \bar{\Omega}, \quad 0<u(x, y) \leqslant C \mathrm{e}^{(y-\phi(x)) c_{0} \sin \alpha},
$$

the function $v$ is well defined. From the Harnack inequality [24] and standard elliptic estimates, there exists then a positive constant $C_{1}$ such that

$$
\|\nabla u(x, y)\| \leqslant C_{1} \mathrm{e}^{(y-\phi(x)) c_{0} \sin \alpha} \text { for all }(x, y) \text { such that } y \leqslant \phi(x)-1 .
$$

Since $u$ is globally Lipschitz in $\mathbb{R}^{2}$, one has

$$
\|\nabla u(x, y)\| \leqslant C_{2} \leqslant C_{2}^{\prime} \mathrm{e}^{(y-\phi(x)) c_{0} \sin \alpha} \text { for all }(x, y) \text { such that } \phi(x)-1 \leqslant y \leqslant \phi(x),
$$

for some positive constants $C_{2}$ and $C_{2}^{\prime}$. Therefore, there exists a positive number $\rho_{1}$ such that

$$
\forall(x, y) \in \bar{\Omega},\|\nabla u(x, y)\| \leqslant \rho_{1} \mathrm{e}^{(y-\phi(x)) c_{0} \sin \alpha} .
$$

By induction and by the standard elliptic estimates up to the boundary, it follows that

$$
\forall n \in \mathbb{N}, \forall(x, y) \in \bar{\Omega},\left\|D^{n} u(x, y)\right\| \leqslant \rho_{n}(x) \mathrm{e}^{(y-\phi(x)) c_{0} \sin \alpha}
$$

where, for every $n \in \mathbb{N}, D^{n} u$ denotes any $n$-order partial derivative of $u$ and $\rho_{n}$ is a function which is locally bounded and depends on $\phi$ only. We repeat here that the functions $\rho_{0}$ and $\rho_{1}$ can be assumed to be globally bounded.

Since $\|\nabla u\| \leqslant \rho_{1}(x) \mathrm{e}^{(y-\phi(x)) c_{0} \sin \alpha}$ in the set $\bar{\Omega}$ and since the function $\phi$ is continuous, it is straightforward to check, by distinguishing left neighbourhoods and right neighbourhoods, that the function $x \mapsto v(x)$ is continuous at any point $x \in \mathbb{R}$. From (2.10), we also have

$$
\forall x \in \mathbb{R}, \quad 0 \leqslant v(x) \leqslant \frac{C}{c_{0} \sin \alpha} .
$$


By applying (2.13) to $\partial_{x x}^{2} u$ and since the function $\phi$ is of class $C^{1}$, we similarily infer that the function $v$ is differentiable and that

$$
\forall x \in \mathbb{R}, \quad v^{\prime}(x)=\phi^{\prime}(x)+\int_{-\infty}^{\phi(x)} \partial_{x} u(x, y) \mathrm{d} y .
$$

This function $v^{\prime}$ is itself continuous. Since the function $\rho_{1}$ is bounded and since $\phi$ is globally Lipschitz-continuous, there then exists a constant $C>0$ such that $\left\|v^{\prime}\right\|_{\infty} \leqslant C$.

The same arguments applied at any order yield that the function $v$ is of class $C^{\infty}(\mathbb{R})$. In particular, we have

$$
\forall x \in \mathbb{R}, \quad v^{\prime \prime}(x)=\phi^{\prime \prime}(x)+\phi^{\prime}(x) \partial_{x} u(x, \phi(x))+\int_{-\infty}^{\phi(x)} \partial_{x x}^{2} u(x, y) \mathrm{d} y .
$$

On the other hand, we know that $\Delta u-c \partial_{y} u=0$ in the set $\bar{\Omega}=\{y \leqslant \phi(x)\}$. Thus

$$
\int_{-\infty}^{\phi(x)} \partial_{x x}^{2} u(x, y) \mathrm{d} y=c[u(x, y)]_{y=-\infty}^{y=\phi(x)}-\left[\partial_{y} u(x, y)\right]_{y=-\infty}^{y=\phi(x)}=c-\partial_{y} u(x, \phi(x)) .
$$

At any point $(x, \phi(x))$, the outside unit normal $n$ to $\bar{\Omega}$ is $n=\frac{1}{\sqrt{1+\phi^{\prime 2}}}\left(-\phi^{\prime}, 1\right)$. Since $\nabla u=$ $\left(\partial_{n} u\right) n=c_{0} n$ at any point $(x, \phi(x))$ (remember that $\nabla u$ stands for the gradient of the restriction of $u$ to the set $\bar{\Omega})$, it follows that

$$
\partial_{x} u(x, \phi(x))=\nabla u \cdot e_{x}=-\frac{c_{0} \phi^{\prime}(x)}{\sqrt{1+\phi^{\prime 2}(x)}}
$$

and that

$$
\partial_{y} u(x, \phi(x))=\nabla u \cdot e_{y}=\frac{c_{0}}{\sqrt{1+\phi^{\prime 2}(x)}} .
$$

Therefore, one obtains

$$
v^{\prime \prime}(x)=c+\phi^{\prime \prime}(x)-\frac{c_{0}}{\sqrt{1+\phi^{\prime 2}(x)}}-\frac{c_{0} \phi^{\prime 2}(x)}{\sqrt{1+\phi^{\prime 2}(x)}}=c+\phi^{\prime \prime}(x)-c_{0} \sqrt{1+\phi^{\prime 2}(x)},
$$

which completes the proof of Lemma 2.7.

Let us now turn to the following lemma.

Lemma 2.8 The function $\phi$ satisfies $\cot \alpha+\phi^{\prime}(x) \geqslant 0$ in $\mathbb{R}^{+}$and

$$
0 \leqslant \int_{0}^{+\infty}\left(\cot \alpha+\phi^{\prime}(x)\right) \mathrm{d} x<+\infty
$$

Proof. By integration of (2.12), we deduce that

$$
v^{\prime}(x)=v^{\prime}(0)+\phi^{\prime}(x)-\phi^{\prime}(0)+\int_{0}^{x}\left(c-c_{0} \sqrt{1+\phi^{\prime 2}(s)}\right) \mathrm{d} s .
$$


Since $u$ and $\phi$ are symmetric in $x$, the function $v$ is even and $v^{\prime}(0)=\phi^{\prime}(0)=0$. Therefore,

$$
\forall x \in \mathbb{R}, \quad \int_{0}^{x}\left(c-c_{0} \sqrt{1+\phi^{\prime 2}(s)}\right) \mathrm{d} s=v^{\prime}(x)-\phi^{\prime}(x) .
$$

Now consider the function

$$
\zeta(x)=c-c_{0} \sqrt{1+\phi^{\prime 2}(x)}=\frac{c_{0}}{\sin \alpha}-c_{0} \sqrt{1+\phi^{\prime 2}(x)} .
$$

It is non-negative because $\left\|\phi^{\prime}\right\| \leqslant \cot \alpha$. Moreover, the function $v^{\prime}-\phi^{\prime}$ is globally bounded because both $v^{\prime}$ and $\phi^{\prime}$ are bounded. That implies that $\zeta \in L^{1}(\mathbb{R})$.

We know that $-\cot \alpha \leqslant \phi^{\prime}(x) \leqslant 0$ for all $x \geqslant 0$. Hence,

$$
\begin{aligned}
\forall x \geqslant 0, \quad 0 \leqslant \cot \alpha+\phi^{\prime}(x) & =\frac{1}{\cot \alpha-\phi^{\prime}(x)}\left(\cot ^{2} \alpha-\phi^{\prime 2}(x)\right) \\
& \leqslant \frac{1}{\cot \alpha}\left(\frac{1}{\sin ^{2} \alpha}-1-{\left.\phi^{\prime 2}(x)\right)}\right. \\
& =\frac{1}{\cot \alpha}\left(\frac{1}{\sin \alpha}-\sqrt{1+\phi^{\prime 2}(x)}\right)\left(\frac{1}{\sin \alpha}+\sqrt{1+\phi^{\prime 2}(x)}\right) \\
& \leqslant \frac{2}{c_{0} \cos \alpha} \zeta(x)
\end{aligned}
$$

because $\sqrt{1+\phi^{\prime 2}} \leqslant 1 / \sin \alpha$. Since $\zeta \in L^{1}(\mathbb{R})$, this gives (2.15).

From Lemma 2.8, the function $x \mapsto x \cot \alpha+\phi(x)$ is nondecreasing in $\mathbb{R}^{+}$and goes to a finite limit $L$ as $x \rightarrow+\infty$. Therefore, since $\phi$ is even, it follows that the free boundary $\Gamma=\{y=\phi(x)\}$ has the two half-lines $\{y=\mp x \cot \alpha+L\}$ as asymptotes as $x \rightarrow \pm \infty$, in the sense that

$$
\phi(x)+|x| \cot \alpha \rightarrow L \text { as } x \rightarrow \pm \infty .
$$

Proof of (1.2). Theorem 2.6 and Lemma 2.8 imply that $\phi^{\prime}(x) \rightarrow-\cot \alpha$ as $x \rightarrow+\infty$. Since $\phi$ is even, this eventually yields (1.2).

This completes the proof of Theorem.

\section{Definition of a class of free boundary problems, monotonicity and regularity results}

The remaining part of this paper is devoted to the proof of Theorem 1.6. In order to study the qualitative properties of the solutions of (1.1)-(1.2), we shall use a comparison principle for suband supersolutions of such free boundary problems. This comparison principle actually requires a uniformity condition on the free boundary. That is why we define in this section a more adapted framework for the free boundary problems we shall deal with.

From now on, $u$ denotes a globally Lipschitz-continuous function on $\mathbb{R}^{2}$ such that $0<u \leqslant 1$. We define

$$
\Omega(u)=\{u<1\}
$$

and

$$
\Gamma(u)=\partial \Omega(u) .
$$

We say that the boundary $\Gamma(u)$ is regular if it satisfies the following definition. 
Definition 3.1 The boundary $\Gamma(u)$ is said to be regular if and only if there exists a constant $\delta>0$ such that for every point $X_{0} \in \Gamma(u)$ there exist two open balls $B^{ \pm}$with radius $\delta$ such that $B^{-} \subset$ $\Omega(u), \partial B^{-} \cap \partial B^{+}=\left\{X_{0}\right\}$ and $B^{+} \cap \mathcal{C}^{-}=\emptyset$, where $\mathcal{C}^{-}$is the connected component of $\Omega \cap B_{2 \delta}\left(X_{0}\right)$ containing $B^{-}$.

Remark 3.2 This regularity notion for the boundary is broad enough to include the situation where an open ball $B$ contains two disjoint connected components of $\Omega(u) \cap B$ separated by a thin piece in $\mathbb{R}^{2} \backslash \Omega(u)$. This notion is also broad enough to be satisfied in the case where $\Gamma(u)$ and the restriction of $u$ to $\overline{\Omega(u)}$ are smooth enough (see Proposition 3.7 below). On the other hand, this notion is strong enough to allow for some monotonicity, compactness results or comparison principles for the functions $u$ (see Theorems 3.4, 4.5 and Proposition 4.2 below).

We shall also use the following notion of weak boundary condition on $\Gamma(u)$ (in the following definition, $\Gamma(u)$ need not be regular).

Definition 3.3 We say that $u$ satisfies

$$
\frac{\partial u}{\partial n}=c_{0} \text { on } \Gamma(u)
$$

if and only if there exist two continuous functions $h_{1}, h_{2}:[0,+\infty) \rightarrow[0,1]$ such that, for each $i=1,2, h_{i}(0)=0,0<h_{i}(t)<1$ for all $t>0, h_{i}$ is differentiable at 0 with $h_{i}^{\prime}(0)=c_{0}$, and

$$
\forall X \in \Omega(u), \quad 1-h_{1}(r) \leqslant u(X) \leqslant 1-h_{2}(r), \text { where } r=d(X, \Gamma(u)) .
$$

The above condition (3.1) contains both local and global information in the sense that it says that the restriction of $u$ to $\overline{\Omega(u)}$ has a uniform first-order Taylor expansion near $\Gamma(u)$ with the slope $c_{0}$ on $\Gamma(u)$. Furthermore, $u$ is bounded away from 0 and 1 at any finite distance of $\Gamma(u)$. Therefore, the above definition is stronger than the weak definitions of the boundary conditions in the sense of Caffarelli.

Let now $\phi: \mathbb{R} \rightarrow \mathbb{R}$ be a given Lipschitz-continuous function, let $c_{1}, c_{2}$ be two given real numbers and, under the above two definitions, let us consider the globally Lipschitz-continuous solutions $u, 0<u \leqslant 1$, of the following free boundary problem:

$$
\left\{\begin{aligned}
L u:=\Delta u+c_{1} \frac{\partial u}{\partial x}+c_{2} \frac{\partial u}{\partial y} & =0 \text { in } \Omega(u)=\{u<1\} \\
\frac{\partial u}{\partial n} & =c_{0} \text { on } \Gamma(u) \text { in the sense of Definition 3.3, } \\
\lim _{y_{0} \rightarrow-\infty} \sup _{\Omega^{-}\left(y_{0}\right)} u & =0 \\
\exists y_{1} \in \mathbb{R}, \quad u & =1 \text { on } \Omega^{+}\left(y_{1}\right)
\end{aligned}\right.
$$

where

$$
\forall y_{0} \in \mathbb{R}, \quad\left\{\begin{array}{l}
\Omega^{-}\left(y_{0}\right)=\left\{y<\phi(x)+y_{0}\right\}, \\
\Omega^{+}\left(y_{0}\right)=\left\{y>\phi(x)+y_{0}\right\} .
\end{array}\right.
$$

Note that, from standard elliptic estimates, the equality $L u=0$ in $\Omega(u)$ holds in the classical sense and that, from the strong maximum principle, any $u$ as above is positive in $\Omega(u)$.

Let us first state the following monotonicity result. 
Theorem 3.4 Let $\phi: \mathbb{R} \rightarrow \mathbb{R}$ be a Lipschitz-continuous function. Let $u$ be a Lipschitz-continuous function such that $0<u \leqslant 1$ in $\mathbb{R}^{2}$ and solving (3.2) in the sense of Definitions 3.1 and 3.3.

Then $u$ is nondecreasing with respect to the variable $y$.

This theorem is proved in Section 4. From Theorem 3.4, a corollary follows immediately.

Corollary 3.5 Let $\phi: \mathbb{R} \rightarrow \mathbb{R}$ be a Lipschitz-continuous function. Let $u$ be a Lipschitz-continuous function such that $0<u \leqslant 1$ in $\mathbb{R}^{2}$ and solving (3.2) in the sense of Definitions 3.1 and 3.3. Assume, moreover, that $\operatorname{Lip}(\phi) \leqslant \cot \beta$ for some $0<\beta \leqslant \pi / 2$.

Then $u$ is nonincreasing in all directions $\tau$ belonging to the cone

$$
\mathcal{C}^{-}(\beta)=\left\{\rho v, \rho>0,\|v\|=1, v_{y} \leqslant-\cos \beta\right\} .
$$

Proof. It immediately follows from Theorem 3.4 in the case $\tau=\rho v$ with $\rho>0$ and $v_{y}<-\cos \beta$ : namely, this is done by rotating the frame and writting an equivalent problem in a new orthonormal frame $\left(e_{x}^{\prime}, e_{y}^{\prime}\right)$ with $e_{y}^{\prime}=v$. The case $v_{y}=-\cos \beta$ then follows by continuity.

Remark 3.6 Under the conditions of Theorem 3.4 and Corollary 3.5, the boundary $\Gamma(u)$ is then a Lipschitz graph with respect to all directions $\tau \in\left(\mathcal{C}^{-}(\beta)\right)^{\mathrm{O}}$, and in particular in the variable $y$. The latter was actually not a priori required in Definitions 3.1 and 3.3.

The above monotonicity results have natural extensions in higher dimensions.

The following proposition makes the link between the classical solutions of (1.1) with smooth boundary and the solutions of (3.2).

Proposition 3.7 Let $u$ be a globally Lipschitz-continuous function in $\mathbb{R}^{2}$ such that $0<u \leqslant 1$, both $\Omega(u)$ and $\mathbb{R}^{2} \backslash \Omega(u)$ are not empty, and the restriction $\tilde{u}$ of $u$ to $\overline{\Omega(u)}$ is $C^{1}$ up to the boundary $\Gamma(u)$. Assume that $\Gamma(u)$ is globally $C^{1,1}$ with bounded curvature. Assume that $u$ solves (1.1), where $\frac{\partial u}{\partial n}=c_{0}$ stands for $\frac{\partial \tilde{u}}{\partial n}=c_{0}$ on $\Gamma(u)$ (in the classical sense) and $n$ is the outward unit normal to $\Omega(u)$ on $\Gamma(u)$.

Then $\Gamma(u)$ is regular in the sense of Definition 3.1 and $\frac{\partial u}{\partial n}=c_{0}$ on $\Gamma(u)$ in the sense of Definition 3.3.

Remark 3.8 Note in particular that, for each $\alpha \in(0, \pi / 2]$, the function $u^{\alpha}$ given in Theorem 1.1 is then a solution of a problem of the type (3.2) in the sense of Definitions 3.1 and 3.3, with $\left(c_{1}, c_{2}\right)=$ $\left(0,-c_{0} / \sin \alpha\right)$ and $\phi=\phi^{\alpha}$.

The following proposition shows the role played by the same topological assumption for $\mathbb{R}^{2} \backslash \Omega(u)$ as in Theorem 1.6.

Proposition 3.9 Given the assumptions of Proposition 3.7, assume moreover that $\mathbb{R}^{2} \backslash \Omega(u)$ has no bounded connected components.

Then there exists a Lipschitz-continuous function $\phi$ such that $\Gamma(u)=\{y=\phi(x), x \in \mathbb{R}\}$. As a consequence, even if it means changing $(c, u, \Omega)$ into $(-c, u(-x,-y),-\Omega)$, then $u$ satisfies (3.2) with $\left(c_{1}, c_{2}\right)=(0,-c)$ and $\Omega(u)=\{y<\phi(x)\}$.

Let us now turn to the proof of Proposition 3.7.

Proof of Proposition 3.7. Step 1: the boundary $\Gamma(u)$ is regular. First of all, it follows from the assumptions of Proposition 3.7 that, at every point $X_{0} \in \Gamma(u)$, there exists a (unique) outward unit 
normal $n_{X_{0}}$ to $\Omega(u)$ and, if $B$ is an open ball such that $X_{0} \in \partial B$ and $B \subset \Omega(u)$, then $n_{X_{0}}$ is the normal vector to $B$ at $X_{0}$.

Now suppose that $\Gamma(u)$ is not regular in the sense of Definition 3.1. Then there exist two sequences $\varepsilon_{n} \rightarrow 0^{+}$and $X_{n} \in \Gamma(u)$ such that either (1) the open ball $B_{n}^{-}$of radius $\varepsilon_{n}$ and centre $X_{n}-\varepsilon_{n} n_{X_{n}}$ is not included in $\Omega(u)$, or (2) $B_{n}^{-} \subset \Omega(u)$ and the connected component of $\Omega(u) \cap B_{2 \varepsilon_{n}}\left(X_{n}\right)$ meets the ball $B_{n}^{+}$of radius $\varepsilon_{n}$ and centre $X_{n}+\varepsilon_{n} n_{X_{n}}$. Case (2) cannot occur for an infinite subsequence $\varepsilon_{n} \rightarrow 0$ since $\Gamma(u)$ is assumed to have a bounded curvature.

Therefore, for $n$ large enough, case (1) occurs and, by using again that $\Gamma(u)$ has a bounded curvature, it follows that, if we denote by $\gamma_{n}$ the connected component of $\Gamma(u) \cap B_{2 \varepsilon_{n}}\left(X_{n}\right)$ containing $X_{n}$ and by $\mathcal{C}_{n}$ the connected component of $B_{2 \varepsilon_{n}}\left(X_{n}\right) \cap \overline{\Omega(u)}$ containing $X_{n}$, then

$$
0<d\left(X_{n},\left(\partial \mathcal{C}_{n}\right) \backslash \gamma_{n}\right)<\varepsilon_{n} .
$$

Let $Y_{n}$ be a point which realizes the minimum of that distance and call

$$
u_{n}(X)=\frac{1-u\left(X_{n}+\left|X_{n}-Y_{n}\right| X\right)}{\left|X_{n}-Y_{n}\right|}, \quad \Omega_{n}=\frac{\Omega(u)-X_{n}}{\left|X_{n}-Y_{n}\right|} .
$$

Since the curvature of $\Gamma(u)$ is bounded, the scalar product $n_{X_{n}} \cdot \frac{Y_{n}-X_{n}}{\left|Y_{n}-X_{n}\right|} \rightarrow-1$ and the curvature of $\partial \Omega_{n}$, which is less that $\left|X_{n}-Y_{n}\right|$ times the curvature of $\Gamma(u)$, goes to 0 as $n \rightarrow+\infty$. On the other hand, since $u_{n}(0)=0$ and since the Lipschitz bound of each $u_{n}$ is not greater than that of $u$, the functions $u_{n}$ are locally bounded and converge locally, up to extraction of some subsequence, to a Lipschitz-continuous function $u_{0}$. Up to a rotation of the frame and by still calling $u_{0}$ the rotation of $u_{0}$, one then has

$$
\left\{\begin{aligned}
\Delta u_{0} & =0 \text { in } \mathcal{D}^{\prime}\left(\Omega_{0}\right), \\
u_{0} & =0 \text { on } \partial \Omega_{0}, \\
u_{0} & \geqslant 0 \text { in } \mathbb{R}^{2},
\end{aligned}\right.
$$

where $\Omega_{0}=\left\{(x, y) \in \mathbb{R}^{2}, 0<y<1\right\}$. The Lipschitz-continuous function $v(y):=$ $\sup _{x \in \mathbb{R}} u_{0}(x, y)$ then satisfies

$$
\left\{\begin{aligned}
v^{\prime \prime} & \geqslant 0 \text { in } \mathcal{D}^{\prime}(0,1), \\
v(0)=v(1) & =0 \\
v & \geqslant 0 \text { in } \mathbb{R} .
\end{aligned}\right.
$$

Therefore, $v \equiv 0$ in $[0,1]$ and $u_{0} \equiv 0$ in $\overline{\Omega_{0}}$.

Now, say in $B_{1 / 2}(0)$, the functions $u_{n}$ are Lipschitz-continuous and $\partial \Omega_{n}$ (where $u_{n}=0$ ) is $C^{1,1}$, with norms independent of $n$. Thus, since $u_{n}(0)=0$, the functions $u_{n}$ are uniformly bounded in $B_{1 / 2}(0)$. It follows then from standard estimates up to the boundary (see Morrey [40]) that the functions $u_{n}$ are in $W^{2, p}\left(\Omega_{n} \cap B_{1 / 2}(0)\right)$ for every $p \in[1,+\infty)$ and that $\left\|u_{n}\right\|_{W^{2, p}\left(\Omega_{n} \cap B_{1 / 2}(0)\right)} \leqslant$ $C(p)$ where $C(p)$ does not depend on $n$. From Sobolev injections,

$$
\left\|u_{n}\right\|_{C^{1, \beta}\left(\overline{\left.\Omega_{n} \cap B_{1 / 2}(0)\right)}\right.} \leqslant C^{\prime}(\beta)
$$

for each $\beta \in[0,1)$. Choose a $\beta \in(0,1)$. From the assumptions of Proposition 3.7, the restrictions $\tilde{u}_{n}$ of the functions $u_{n}$ on $\overline{\Omega_{n}}$ satisfy $\partial_{n} \tilde{u}_{n}=-c_{0}$ on $\partial \Omega_{n}$ in the classical sense. Therefore, there exists a constant $C$ such that, for all $n$ large enough and for all $r \in(0,1 / 2)$,

$$
-r n_{X_{n}} \in \Omega_{n} \text { and }\left|u_{n}\left(-r n_{X_{n}}\right)-c_{0} r\right| \leqslant C r^{1+\beta} \text {. }
$$


Since $n_{X_{n}} \cdot \frac{Y_{n}-X_{n}}{\left|n_{n}-X_{n}\right|} \rightarrow-1$, the passage to the limit $n \rightarrow+\infty$ in the above inequality contradicts the fact that $u_{0} \equiv 0$ in $\Omega_{0}$.

As a consequence, the boundary $\Gamma(u)$ is regular in the sense of Definition 3.1 . by

Step 2: $u$ satisfies $\frac{\partial u}{\partial n}=c_{0}$ in the sense of Definition 3.3. Let us define the functions $h_{1}$ and $h_{2}$

$$
\left\{\begin{array}{l}
h_{1}(t)=\sup _{X \in \Omega(u),} \operatorname{dinf}_{X(u))=t}(1-u(X)) \\
h_{2}(t)=\inf _{X \in \Omega(u), d(X, \Gamma(u))=t}(1-u(X))
\end{array}\right.
$$

and let us prove that both $h_{1}$ and $h_{2}$ satisfy the requirements of Definition 3.3.

First of all, one immediately has $0 \leqslant h_{2} \leqslant h_{1} \leqslant 1$ since $0<u \leqslant 1$. Moreover, (3.1) follows from the definitions of $h_{1}$ and $h_{2}$.

Next, let us observe that, from step 1 (namely, the free boundary $\Gamma(u)$ is regular), there exists $\delta>0$ such that

$$
\forall X_{0} \in \Gamma(u), \forall r \in[0, \delta], \quad d\left(X_{0}-r n_{X_{0}}, \Gamma(u)\right)=r .
$$

Furthermore, if $X \in \Omega(u)$ is such that $d(X, \Gamma(u))=r \in[0, \delta]$, then there exists $X_{0} \in \Gamma(u)$ such that $X=X_{0}-r n_{X_{0}}$. Therefore,

$$
\forall r \in[0, \delta],\left\{\begin{array}{l}
h_{1}(r)=\sup _{X_{0} \in \Gamma(u)}\left(1-u\left(X_{0}-r n_{X_{0}}\right)\right) \\
h_{2}(r)=\inf _{X_{0} \in \Gamma(u)}\left(1-u\left(X_{0}-r n_{X_{0}}\right)\right) .
\end{array}\right.
$$

On the other hand, since $\Gamma(u)$ is globally $C^{1,1}$ by hypothesis and since $u$ is globally bounded, it follows as above from regularity theory for elliptic equations [40] that the restriction $\tilde{u}$ of $u$ in $\overline{\Omega(u)}$ is actually globally $C^{1, \beta}$ in $\overline{\Omega(u)}$ for every $\beta \in[0,1)$. Choose an arbitrary $\beta \in(0,1)$. Since $\partial_{n} \tilde{u}=c_{0}$ on $\Gamma(u)$ in the classical sense, there exist then two positive constants $r_{0}$ and $C$ such that

$$
\forall 0 \leqslant r \leqslant r_{0}, \quad \forall X_{0} \in \Gamma(u), \quad\left|1-c_{0} r-u\left(X_{0}-r n_{X_{0}}\right)\right| \leqslant C r^{1+\beta} .
$$

From (3.4), it is then found that

$$
\left|\frac{h_{1}(r)-c_{0} r}{r}\right|,\left|\frac{h_{2}(r)-c_{0} r}{r}\right| \leqslant C r^{\beta}
$$

for $r$ small enough. Eventually, $h_{1}^{\prime}(0)=h_{2}^{\prime}(0)=c_{0}$. Since $h_{1} \geqslant h_{2}$ and $h_{1}(0)=h_{2}(0)=0$, that especially yields the existence of a $\delta_{0}>0$ such that

$$
\left\{\begin{array}{l}
\inf _{\left[\eta, \delta_{0}\right]} h_{1} \geqslant \inf _{\left[\eta, \delta_{0}\right]} h_{2}>0 \text { for all } 0<\eta \leqslant \delta_{0}, \\
1>\sup _{\left[0, \delta_{0}\right]} h_{1} \geqslant \sup _{\left[0, \delta_{0}\right]} h_{2} .
\end{array}\right.
$$

Lastly, one shall show that $\inf _{[a, b]} h_{2}>0$ and $\sup _{[a, b]} h_{1}<1$ for all $0<a \leqslant b<+\infty$. Assuming that has been proved, then, even if it means changing the functions $h_{1}$ and $h_{2}$ (increasing $h_{1}$ and decreasing $\left.h_{2}\right), h_{1}$ and $h_{2}$ can be assumed to be continuous on $[0,+\infty)$ and to satisfy all other requirements of Definition 3.3. 
To begin with, let us prove that

$$
\inf _{[a, b]} h_{2}>0 \text { for all } 0<a \leqslant b<+\infty .
$$

Without loss of generality we can assume that $a \geqslant \delta_{0}$ because of (3.5). Suppose now by contradiction that $\inf _{[a, b]} h_{2}=0$ for some $0<\delta_{0} \leqslant a \leqslant b<+\infty$. There exist then a sequence $t_{k} \rightarrow t \in[a, b]$ and a sequence of points $X_{k} \in \Omega(u)$ such that $d\left(X_{k}, \Gamma(u)\right)=t_{k}$ and $u\left(X_{k}\right) \rightarrow 1$ as $k \rightarrow+\infty$. Let $Y_{k}$ be on $\partial B_{t_{k}}\left(X_{k}\right) \cap \Gamma(u)$. One has $X_{k}=Y_{k}-t_{k} n_{Y_{k}}$. Up to extraction of some subsequence, one can assume that $n_{Y_{k}} \rightarrow n_{\infty}$ and, since $u$ is globally bounded and Lipschitzcontinuous in $\mathbb{R}^{2}$, one can assume that the functions $u_{k}(X)=u\left(X_{k}+X\right)$ converge locally uniformly in $\mathbb{R}^{2}$ to a globally Lipschitz-continuous function $u_{\infty}$ such that

$$
\left\{\begin{aligned}
\Delta u_{\infty}-c \partial_{y} u_{\infty} & =0 \text { in } \mathcal{D}^{\prime}\left(B_{t}(0)\right), \\
u_{\infty} & \leqslant 1 \text { in } \mathbb{R}^{2}, \\
u_{\infty}(0)=1 &
\end{aligned}\right.
$$

The strong maximum principle then yields $u_{\infty} \equiv 1$ in $\overline{B_{t}(0)}$. On the other hand, the points $Z_{k}=$ $Y_{k}-\delta_{0} n_{Y_{k}}$ are in $B_{t_{k}}\left(X_{k}\right) \subset \Omega(u)$ (because $t \geqslant a \geqslant \delta_{0}$ and $\delta_{0}<2 t_{k}$ for $k$ large enough) and these points are such that $d\left(Z_{k}, \Gamma(u)\right)=\delta_{0}$. Hence,

$$
1-u_{k}\left(\left(t_{k}-\delta_{0}\right) n_{Y_{k}}\right)=1-u\left(Z_{k}\right) \geqslant h_{2}\left(d\left(Z_{k}, \Gamma(u)\right)\right)=h_{2}\left(\delta_{0}\right)>0
$$

from (3.5). The passage to the limit $k \rightarrow+\infty$ yields

$$
1-u_{\infty}\left(\left(t-\delta_{0}\right) n_{\infty}\right) \geqslant h_{2}\left(\delta_{0}\right)>0,
$$

whence $u_{\infty}\left(\left(t-\delta_{0}\right) n_{\infty}\right)<1$. Since $\left(t-\delta_{0}\right) n_{\infty} \in B_{t}(0)$, one has reached a contradiction.

Similarly, suppose by contradiction that $\sup _{[a, b]} h_{1}=1$ for some $0<a \leqslant b<+\infty$. After a change of the origin and a passage to the limit, one is led as above to the existence of a globally Lipschitz-continuous function $v_{\infty}$ in $\mathbb{R}^{2}$ such that

$$
\left\{\begin{aligned}
\Delta v_{\infty}-c \partial_{y} v_{\infty} & =0 \text { in } \mathcal{D}^{\prime}\left(B_{t}(0)\right), \\
v_{\infty} & \geqslant 0 \text { in } \mathbb{R}^{2} \\
v_{\infty}(0) & =0
\end{aligned}\right.
$$

where $0<t<+\infty$. From the strong maximum principle, the function $v_{\infty}$ is then identically equal to 0 in $\overline{B_{t}(0)}$. Under the notation of the previous paragraph, one has $Y_{k} \in \Gamma(u)$, whence $u_{k}\left(t_{k} n_{Y_{k}}\right)=1$ and $v_{\infty}\left(t n_{\infty}\right)=1$ at the limit (the functions $u_{k}$ are uniformly and globally Lipschitzcontinuous and locally converge to $v_{\infty}$ ). The latter is in contradiction with the fact that $v_{\infty}=0$ in $\overline{B_{t}(0)}$.

That completes the proof of Proposition 3.7.

To complete this section, let us turn to the proof of Proposition 3.9.

Proof of Proposition 3.9. For the sake of clarity, the proof is divided into several lemmas. In what follows, one makes the assumptions of Propositions 3.9.

Lemma 3.10 If $c \geqslant 0$ and if there exist an open ball $B$ and a function $\phi$ such that $\gamma=\{y=$ $\phi(x)\} \cap B \subset \Gamma(u)$ and the outward unit normal to $\Omega(u)$ on $\gamma$ has a nonpositive $y$-component, then the set $\{y \leqslant \phi(x)\} \cap B$ is convex. 
Proof. Let us first notice that the function $u$ is actually of class $C^{\infty}$ in $\Omega(u)$, and, from regularity results of Kinderlehrer $e t a l$. [32], the boundary $\Gamma(u)$ is even analytic and the restriction $\tilde{u}$ of $u$ in $\overline{\Omega(u)}$ is analytic.

Since $\Delta u-c \partial_{y} u=0$ in $\Omega(u)$, it is found that

$$
\Delta\left(|\nabla u|^{2}\right)-c \partial_{y}\left(|\nabla u|^{2}\right)=2\left[\left(\partial_{x x}^{2} u\right)^{2}+2\left(\partial_{x y}^{2} u\right)^{2}+\left(\partial_{y y}^{2} u\right)^{2}\right]=2\left|D^{2} u\right|^{2} \geqslant 0 \text { in } \Omega(u) .
$$

Moreover $u \rightarrow 0$ as $d(X, \Gamma(u)) \rightarrow+\infty$ and $X \in \Omega(u)$, whence $|\nabla u| \rightarrow 0$ as $d(X, \Gamma(u)) \rightarrow+\infty$. From the maximum principle, the maximum of $|\nabla \tilde{u}|^{2}$ is reached on the boundary $\Gamma(u)$, i.e. $|\nabla \tilde{u}| \leqslant$ $c_{0}$ in $\overline{\Omega(u)}$, and the strong Hopf lemma yields that

$$
D_{n n}^{2} \tilde{u}>0 \text { on } \Gamma(u),
$$

where $n$ is the outward unit normal to $\Omega(u)$ and, for any unit vector $\sigma=\left(\sigma_{x}, \sigma_{y}\right) \in S^{1}$ and any point $(x, y) \in \overline{\Omega(u)}$,

$$
D_{\sigma \sigma}^{2} \tilde{u}(x, y):=\sigma_{x}^{2} \partial_{x x}^{2} \tilde{u}(x, y)+2 \sigma_{x} \sigma_{y} \partial_{x y}^{2} \tilde{u}(x, y)+\sigma_{y}^{2} \partial_{y y}^{2} \tilde{u}(x, y) .
$$

Therefore, calling $\tau=-n^{\perp}=\left(n_{y},-n_{x}\right)$, it follows that

$$
D_{\tau \tau}^{2} \tilde{u}=c \partial_{y} \tilde{u}-D_{n n}^{2} \tilde{u}<0 \text { on } \gamma
$$

since $c \geqslant 0$ and $\partial_{y} \tilde{u} \leqslant 0$ on $\gamma$ (the latter follows from $\nabla \tilde{u}=c_{0} n$ and $n_{y} \leqslant 0$ on $\gamma$ by assumption). Then, the curvature $K=\frac{D_{\tau}^{2} \tilde{u}}{|\bar{v} \tilde{u}|}$ of $\gamma$ is negative (with the convention that the curvature of the boundary of a disc is positive). The conclusion of Lemma 3.10 follows.

Remark 3.11 The assumption $n_{y} \leqslant 0$ on $\gamma$ means that $\Omega(u)$ is locally above $\gamma$. Note however that $\Omega(u)$ may also meet the set $\{y<\phi(x)\}$.

Lemma 3.12 If $c \geqslant 0$ and if there exists a connected graph $\gamma=\{y=\phi(x), a<x<b\}$ with $-\infty \leqslant a<b \leqslant+\infty$ such that $\gamma \subset \Gamma(u)$ and the outward unit normal to $\Omega(u)$ on $\gamma$ has a nonpositive $y$-component, then $a \neq-\infty$ and $b \neq+\infty$.

Proof. Suppose by contradiction that, say, $a=-\infty$ (the case $b=+\infty$ can be treated similarly). Then there exist a sequence $x_{k} \rightarrow-\infty$ and some open balls $B_{k}$ of radius $k$ such that

$$
B_{k} \subset\{y<\phi(x)\} \text { and } X_{k}=\left(x_{k}, \phi\left(x_{k}\right)\right) \in \partial B_{k} \cap \gamma .
$$

Note that since $\gamma$ is connected, each ball $B_{k}$ can be defined for $k$ large enough such that $-k<b$ as the ball of radius $k$ and centre $\left(-2 k, t_{k}\right)$, where

$$
t_{k}=\sup \left\{t, B_{k}(-2 k, t) \subset\{y<\phi(x)\}\right\}
$$

and $B_{k}(-2 k, t)$ is the open ball of radius $k$ and centre $(-2 k, t)$.

Up to extraction of some subsequence, the functions $u_{k}(X)=u\left(X_{k}+X\right)$ locally converge to a globally Lipschitz-continuous function $u_{\infty}$. Since $\partial_{n} \tilde{u}=c_{0}>0$ on $\Gamma(u)$, it immediately follows that $u_{\infty}$, as $u$, satisfies

$$
\Delta u_{\infty}-c \partial_{y} u_{\infty} \leqslant 0 \text { in } \mathcal{D}^{\prime}\left(\mathbb{R}^{2}\right)
$$


and $0 \leqslant u_{\infty} \leqslant 1$ in $\mathbb{R}^{2}$. One can also assume that the unit outward normals $n_{k}$ to $\Omega(u)$ at $\left(x_{k}, \phi\left(x_{k}\right)\right)$ converge to a unit vector $v=\left(v_{x}, v_{y}\right)$. Since the $y$-component of $n_{k}$ is nonpositive, it follows that $v_{y} \leqslant 0$.

On the other hand, it uses from Proposition 3.7 that $\Gamma(u)$ is regular in the sense of Definition 3.1. Let $\delta>0$ be as in Definition 3.1. Let $X \in \mathbb{R}^{2}$ be any point such that $-\delta<X \cdot v<0$. Lemma 3.10 yields that

$$
\gamma \subset\left\{\left(x_{k}+x, \phi\left(x_{k}\right)+y\right) \text { such that }(x, y) \cdot n_{k} \geqslant 0, x<b-x_{k}\right\} .
$$

Since $\left|b-x_{k}\right| \rightarrow+\infty$ and $B_{k} \cap \gamma=\emptyset$, it then follows that there are some points $Y_{k} \in \mathbb{R}^{2}$ such that

$$
X_{k}+Y_{k} \in \gamma, \quad Y_{k} \rightarrow X+|X \cdot v| v \text { and } n_{X_{k}+Y_{k}} \rightarrow v \text { as } k \rightarrow+\infty \text {. }
$$

From Definition 3.1, the ball of radius $\delta$ and centre $Y_{k}-\delta n_{X_{k}+Y_{k}}$ is included in $\Omega(u)$. Therefore,

$$
d\left(X_{k}+X, \Gamma(u)\right) \rightarrow|X \cdot v| \text { as } k \rightarrow+\infty .
$$

It also follows from Proposition 3.7 that $u$ satisfies $\partial_{n} u=c_{0}$ on $\Gamma(u)$ in the sense of Definition 3.3. Thus,

$$
1-h_{1}\left(d\left(X_{k}+X, \Gamma(u)\right) \leqslant u\left(X_{k}+X\right) \leqslant 1-h_{2}\left(d\left(X_{k}+X, \Gamma(u)\right)\right.\right.
$$

and

$$
1-h_{1}(|X \cdot v|) \leqslant u_{\infty}(X) \leqslant 1-h_{2}(|X \cdot v|)
$$

at the limit. That especially implies that $u_{\infty}(X)=1$ for all $X \in \mathbb{R}^{2}$ such that $X \cdot v=0$.

Finally, the function

$$
v\left(y^{\prime}\right):=\inf _{x^{\prime} \in \mathbb{R}} u_{\infty}\left(y^{\prime} v+x^{\prime} v^{\perp}\right)
$$

is globally Lipschitz-continuous on $\mathbb{R}$ and satisfies

$$
\left\{\begin{aligned}
0 \leqslant v & \leqslant 1, \\
v(0) & =1, \\
v\left(y^{\prime}\right) \leqslant 1-h_{2}\left(\left|y^{\prime}\right|\right) & <1 \text { for all }-\delta \leqslant y^{\prime}<0, \\
v^{\prime \prime}-c v_{y} v^{\prime} & \leqslant 0 \text { in } \mathcal{D}^{\prime}(\mathbb{R}) .
\end{aligned}\right.
$$

Choose a barrier subsolution like

$$
w\left(y^{\prime}\right)=(1+\varepsilon) e^{\varepsilon y^{\prime}}-\varepsilon \text { in }(-\infty, 0],
$$

with $\varepsilon>0$. We can check immediately that

$$
w^{\prime \prime}-c v_{y} w^{\prime}=(1+\varepsilon)\left(\varepsilon^{2}-c v_{y} \varepsilon\right) \mathrm{e}^{\varepsilon y^{\prime}} \geqslant 0 \text { in }(-\infty, 0]
$$

since $c \geqslant 0$ and $v_{y} \leqslant 0$. The maximum principle and the passage to the limit $\varepsilon \rightarrow 0^{+}$imply that $v \geqslant 1$ in $(-\infty, 0]$. This contradicts the fact that $v$ is less than 1 in a left neighbourhood of 0 , which completes the proof of Lemma 3.12.

The analogue of the convexity result of Lemma 3.10, in the case where $\Gamma(u)$ has a vertical tangent, is the following lemma.

Lemma 3.13 (i) If $c \geqslant 0$ and if $\Gamma(u)$ has a vertical tangent at a point $X_{0}=\left(x_{0}, y_{0}\right)$ with $n_{X_{0}}=$ $(1,0)$, then $\Gamma(u) \cap B_{r}\left(X_{0}\right) \subset\left\{x>x_{0}\right\} \cup\left\{X_{0}\right\}$ for all $r$ small enough.

(ii) If $c \geqslant 0$ and if $\Gamma(u)$ has a vertical tangent at a point $X_{0}=\left(x_{0}, y_{0}\right)$ with $n_{X_{0}}=(-1,0)$, then $\Gamma(u) \cap B_{r}\left(X_{0}\right) \subset\left\{x<x_{0}\right\} \cup\left\{X_{0}\right\}$ for all $r$ small enough. 
Proof. Up to translation and symmetry with respect to $x$, it is sufficient to prove part (i) with $X_{0}=0$.

In a neighbourhood of 0 , the free boundary $\Gamma(u)$ can be written as a graph $\{x=\psi(y)\}$ where $\psi$ is analytic and $\psi(0)=\psi^{\prime}(0)=0$. Since the connected components of $\Gamma(u)$ cannot have finite endpoints, it follows that if $\psi \equiv 0$ locally then the whole line $\gamma:=\{x=0\}$ is included in $\Gamma(u)$ with $n=(1,0)$ on $\gamma$. As in the proof Lemma 3.12, one can prove that the function $v(x):=\inf _{y \in \mathbb{R}} u(x, y)$ is globally Lipschitz-continuous and satisfies

$$
\left\{\begin{aligned}
0 \leqslant v & \leqslant 1 \\
v(0) & =1 \\
v(x) \leqslant 1-h_{2}(|x|) & <1 \text { for all }-\delta \leqslant x<0 \\
v^{\prime \prime} & \leqslant 0 \text { in } \mathcal{D}^{\prime}(\mathbb{R}) .
\end{aligned}\right.
$$

One is then led to a contradiction as above in Lemma 3.12.

Therefore, near $0, \psi(y)=\alpha y^{p}(1+o(y))$ for some $p \geqslant 2$ and $\alpha \neq 0$. After a simple computation, it is found that

$$
\begin{aligned}
& \partial_{y} \tilde{u}=-c_{0} \frac{\psi^{\prime}}{\sqrt{1+\psi^{\prime 2}}}=-c_{0} \alpha p y^{p-1}(1+o(y)), \\
& -K=\left(\frac{\psi^{\prime}}{\sqrt{1+\psi^{\prime 2}}}\right)^{\prime}=\alpha p(p-1) y^{p-2}(1+o(y))
\end{aligned}
$$

on $\Gamma(u)$ near 0 . Under the notation of Lemma 3.10, (3.6) yields $\partial_{x x}^{2} \tilde{u}=D_{n n}^{2} \tilde{u}>0$ at 0 . Since $\partial_{y} \tilde{u}=0$ at 0 , one gets that

$$
D_{\tau \tau}^{2} \tilde{u}=\partial_{y y}^{2} \tilde{u}=c \partial_{y} \tilde{u}-\partial_{x x}^{2} \tilde{u}<0 \text { at } 0 .
$$

Eventually, $K<0$ at 0 , whence $p=2$ and $\alpha>0$. That completes the proof of Lemma 3.13.

Lemma 3.14 Under the assumptions of Lemma 3.12, the set $\mathbb{R}^{2} \backslash \Omega(u)$ has a bounded connected component.

Proof. Let $(a, b)$ the maximal interval such that $\gamma=\{y=\phi(x), a<x<b\} \subset \Gamma(u)$ is a graph with $\Omega(u)$ locally above $\gamma$. Lemma 3.10 implies that $\phi$ is concave and Lemma 3.12 yields that $-\infty<a<b<+\infty$. Therefore, near each point $a$ and $b, \phi$ is either locally bounded or goes to $-\infty\left(\right.$ as $x \rightarrow a^{+}$or $\left.x \rightarrow b^{-}\right)$.

If, say, $\phi(x) \rightarrow-\infty$ as $x \rightarrow a^{+}$, then, as in the proof of Lemma 3.12 and after a change of the origin and a passage to the limit, there would exist a globally Lipschitz-continuous function $0 \leqslant u_{\infty} \leqslant 1$ such that

$$
\Delta u_{\infty}-c \partial_{y} u_{\infty} \leqslant 0 \text { in } \mathcal{D}^{\prime}\left(\mathbb{R}^{2}\right),
$$

$u_{\infty}(0, y)=1$ for all $y \in \mathbb{R}$ and $u_{\infty}(x, y) \leqslant 1-h_{2}(|x|)$ for all $x \in[-\delta, 0]$ and $y \in \mathbb{R}$. An application of the maximum principle to the function $v(x)=\inf _{y \in \mathbb{R}} u_{\infty}(x, y)$ then leads to a contradiction as in Lemmas 3.12 and 3.13.

Similarly, one can prove that the case $\phi(x) \rightarrow-\infty$ as $x \rightarrow b^{-}$is impossible. Therefore, the concave function $\phi$ can be extended by continuity at $a$ and $b$. From the maximality of the interval $(a, b)$ and the concavity of the function $\phi$, it follows that $\gamma=\{y=\phi(x), a<x<b\}$ has two 
vertical tangents at $A=(a, \phi(a))$ and $B=(b, \phi(b))$ and that $\phi$ is greater than $\phi(a)$ (resp. $\phi(b))$ in an open right (resp. left) neighbourhood of $a$ (resp. $b$ ). Since the connected components of $\Gamma(u)$ cannot have finite endpoints, one concludes from Lemma 3.13 that there are two curves $\gamma_{a}$ and $\gamma_{b}$ included in $\Gamma(u)$, which respectively have $A$ and $B$ as endpoints and which are respectively included in $\{x>a\} \cap\{y<\phi(a)\}$ and $\{x<b\} \cap\{y<\phi(b)\}$, respectively near $A$ and $B$.

Let $\Gamma_{a}$ and $\Gamma_{b}$ be the connected components of $\Gamma(u) \backslash \bar{\gamma}$ containing $\gamma_{a}$ and $\gamma_{b}$. Since the connected components of $\Gamma(u)$ cannot have finite endpoints, the curves $\gamma_{a}$ and $\gamma_{b}$ can then be parametrized by $C^{1}$ functions $X_{a}(t)=\left(x_{a}(t), y_{a}(t)\right)$ and $X_{b}(t)=\left(x_{b}(t), y_{b}(t)\right)$ respectively, defined on $(0,1)$ and such that

$$
X_{a}(t) \rightarrow A \text { and } X_{b}(t) \rightarrow B \text { as } t \rightarrow 0^{+} .
$$

From the definition of $\gamma_{a}$, there exists $\eta>0$ such that $x_{a}(t)>a$ for $t \in(0, \eta)$. Since $\Gamma_{a}$ cannot cross $\bar{\gamma}$ and since the connected components of $\Gamma(u)$ cannot have finite endpoints, one of the following situations necessarily occurs:

(i) there exists $t_{0} \in(0,1)$ such that $x_{a}\left(t_{0}\right)=a, y_{a}\left(t_{0}\right)<\phi(a)$, and $a<x_{a}(t)<b, y_{a}(t)<$ $\phi\left(x_{a}(t)\right)$ for all $t \in\left(0, t_{0}\right)$,

(ii) $a<x_{a}(t)<b, y_{a}(t)<\phi\left(x_{a}(t)\right)$ for all $t \in(0,1)$ and $y_{a}(t) \rightarrow-\infty$ as $t \rightarrow 1^{-}$,

(iii) there exists $t_{0} \in(0,1)$ such that $x_{a}\left(t_{0}\right)=b, y_{a}\left(t_{0}\right)<\phi(b)$, and $a<x_{a}(t)<b, y_{a}(t)<$ $\phi\left(x_{a}(t)\right)$ for all $t \in\left(0, t_{0}\right)$,

(iv) $a<x_{a}(t)<b, y_{a}(t)<\phi\left(x_{a}(t)\right)$ for all $t \in(0,1)$ and $X_{a}(t) \rightarrow B$ as $t \rightarrow 1^{-}$.

One shall now prove that only case (iv) may occur. If case (i) occurs, there exists $t_{1} \in\left(0, t_{0}\right)$ such that $n_{X_{a}\left(t_{1}\right)}=(1,0)$ and $\Gamma_{a}$ is locally included in $\left\{x \leqslant x_{a}\left(t_{1}\right)\right\}$ near $X_{a}\left(t_{1}\right)$ ( $t_{1}$ can be chosen such that $\left.x_{a}\left(t_{1}\right)=\max _{t \in\left(0, t_{0}\right)} x_{a}(t)\right)$. That contradicts Lemma 3.13.

If case (ii) occurs, the same argument as for in case (i) implies that

$$
x_{a}(t) \rightarrow M:=\sup _{t \in(0,1)} x_{a}(t) \in(a, b] \text { as } t \rightarrow 1^{-} .
$$

Therefore, $X_{a}(t) \rightarrow(M,-\infty)$ as $t \rightarrow 1^{-}$. Choose any sequence $t_{n} \rightarrow 1^{-}$. As in the proof of Lemma 3.12, the functions $u_{n}(X)=u\left(X_{a}\left(t_{n}\right)+X\right)$ converge locally, up to extraction of some subsequence, to a globally Lipschitz-continuous function $u_{\infty}(x, y)$ such that

$$
\Delta u_{\infty}-c \partial_{y} u_{\infty} \leqslant 0 \text { in } \mathcal{D}^{\prime}\left(\mathbb{R}^{2}\right),
$$

$0 \leqslant u_{\infty} \leqslant 1, u_{\infty}(0, y)=1$ for all $y \in \mathbb{R}$ and $u(x, y) \leqslant 1-h_{2}(|x|)$ for all $x \in[-\delta, 0]$ and $y \in \mathbb{R}$. Applying the maximum principle to the function $v(x)=\inf _{y \in \mathbb{R}} u_{\infty}(x, y)$ leads to a contradiction as in Lemma 3.13.

If case (iii) occurs, then there exists $t_{1} \in(0,1)$ such that $n_{X_{b}\left(t_{1}\right)}=(-1,0)$ and $\Gamma_{b}$ is locally included in $\left\{x \geqslant x_{b}\left(t_{1}\right)\right\}$ near $X_{b}\left(t_{1}\right)$. That contradicts Lemma 3.13.

Therefore, only case (iv) may occur, which means that $\Gamma_{a}=\Gamma_{b}$ and $\bar{\gamma} \cup \Gamma_{a}$ is a bounded connected component of $\Gamma(u)$. Since $\Omega(u)$ is locally above $\gamma=\{y=\phi(x), a<x<b\}$ and $\Gamma_{a} \subset\{y<\phi(x), a<x<b\}$, one concludes that $\mathbb{R}^{2} \backslash \Omega(u)$ has a bounded connected component.

Lemma 3.15 If $c \geqslant 0$ and $\mathbb{R}^{2} \backslash \Omega(u)$ has no bounded connected components, then there exists a Lipschitz-continuous $\phi: \mathbb{R} \rightarrow \mathbb{R}$ such that $\Omega(u)=\{y<\phi(x)\}$. Moreover, $u$ satisfies (3.2) with $\left(c_{1}, c_{2}\right)=(0,-c)$. 
Proof. From Lemmas 3.13 and 3.14, we deduce that $\Omega(u)$ is a subgraph $\Omega(u)=\{y<\phi(x), a<$ $x<b\}$ where $-\infty \leqslant a<b \leqslant+\infty$ and $\phi$ is a continuous function on $(a, b)$; furthermore, $\Gamma(u)=\{y=\phi(x), a<x<b\}$ has no vertical tangent.

We now claim that

$$
\sup _{\left|x-x^{\prime}\right| \leqslant 1, a<x, x^{\prime}<b}\left|\phi(x)-\phi\left(x^{\prime}\right)\right|<+\infty .
$$

Otherwise, for each $n \geqslant 1$, there are some $a<x_{1, n}<x_{4, n}<b$ such that

$$
x_{4, n}-x_{1, n} \leqslant 1 \text { and }\left|\phi\left(x_{1, n}\right)-\phi\left(x_{4, n}\right)\right| \geqslant n^{2} .
$$

Therefore, there are some $x_{2, n}<x_{3, n} \in\left[x_{1, n}, x_{4, n}\right]$ such that

$$
x_{3, n}-x_{2, n} \leqslant 1 / n \text { and }\left|\phi\left(x_{2, n}\right)-\phi\left(x_{3, n}\right)\right| \geqslant n .
$$

There then exists a sequence of points $X_{n}=\left(x_{n}, \phi\left(x_{n}\right)\right) \in \Gamma(u)$ such that

$$
x_{n} \in\left[x_{2, n}, x_{3, n}\right] \text { and } \phi\left(x_{n}\right)=\left(\phi\left(x_{2, n}\right)+\phi\left(x_{3, n}\right)\right) / 2 .
$$

As in the proof of Lemma 3.14 case (ii) and since $\Gamma(u)$ is globally $C^{1,1}$, it follows that, at least for some subsequence and up to a symmetry $x \rightarrow-x$, the functions $u\left(X_{n}+X\right)$ locally converge to a globally Lipschitz-continuous function $u_{\infty}$ such that

$$
\Delta u_{\infty}-c \partial_{y} u_{\infty} \leqslant 0 \text { in } \mathcal{D}^{\prime}\left(\mathbb{R}^{2}\right)
$$

$0 \leqslant u_{\infty} \leqslant 1, u_{\infty}(0, y)=1$ for all $y \in \mathbb{R}$ and $u_{\infty}(x, y) \leqslant 1-h_{2}(|x|)$ for all $x \in[-\delta, 0]$ and $y \in \mathbb{R}$. By considering the function $v(x)=\inf _{y \in \mathbb{R}} u_{\infty}(x, y)$, one reaches a contradiction as in Lemma 3.13.

Therefore,

$$
\sup _{\left|x-x^{\prime}\right| \leqslant 1, a<x, x^{\prime}<b}\left|\phi(x)-\phi\left(x^{\prime}\right)\right|<+\infty
$$

and, since the graph of $\phi$ cannot have finite endpoints, it follows that $a=-\infty$ and $b=+\infty$. There then exists a globally Lipschitz-continuous function $\psi: \mathbb{R} \rightarrow \mathbb{R}$ and two real numbers $\tau_{0}<\tau_{1}$ such that

$$
\psi(x)+\tau_{0} \leqslant \phi(x) \leqslant \psi(x)+\tau_{1} \text { for all } x \in \mathbb{R} .
$$

Moreover, because $\limsup _{d(X, \Gamma(u)) \rightarrow+\infty, X \in \Omega(u)} u(X)=0$ (remember that $u$ solves (1.1)), we deduce that

$$
\lim _{y_{0} \rightarrow-\infty} \sup _{\Omega^{-}\left(y_{0}\right)} u=0, \text { where } \Omega^{-}\left(y_{0}\right)=\left\{y<\psi(x)+y_{0}\right\} .
$$

The function $u$ then satisfies a problem of the type (3.2) with $\left(c_{1}, c_{2}\right)=(0,-c)$. Lastly, since the function $\psi$ is Lipschitz-continuous with, say, $\operatorname{Lip}(\psi) \leqslant \cot \beta$ and $\beta \in(0, \pi / 2]$, Corollary 3.5 implies that $u$ is nonincreasing in all directions of the cone $\mathcal{C}^{-}(\beta)$. As a consequence, the function $\phi$ itself is globally Lipschitz-continuous and $\operatorname{Lip}(\phi) \leqslant \cot \beta$.

Finally, Lemma 3.15 completes the proof of Proposition 3.9. 


\section{Comparison principle for sub- and supersolutions of a class of free boundary problems}

In order to prove the uniqueness results of Theorems 1.3 and 1.6, we will use a sliding method and we need a comparison principle for sub- and super-solutions of free boundary problems of the type (3.2).

Namely, under the notation in (3.2) and given a Lipschitz-continuous function $\phi: \mathbb{R} \rightarrow \mathbb{R}$, we say that a globally Lipschitz-continuous function $\bar{u}$ (resp. $\underline{u})$ such that $0<\bar{u} \leqslant 1($ resp. $0<\underline{u} \leqslant 1)$ in $\mathbb{R}^{2}$ is a supersolution (resp. subsolution) of problem (3.2) if

$$
\left\{\begin{aligned}
L \bar{u} & \leqslant 0 \text { in } \mathcal{D}^{\prime}(\Omega(\bar{u})), \\
\frac{\partial \bar{u}}{\partial n} & =c_{0} \text { on } \Gamma(\bar{u}) \text { in the sense of Definition 3.3, } \\
\exists y_{1} \in \mathbb{R}, \bar{u} & =1 \text { in } \Omega^{+}\left(y_{1}\right)
\end{aligned}\right.
$$

respectively

$$
\left\{\begin{aligned}
L \underline{u} & \geqslant 0 \text { in } \mathcal{D}^{\prime}(\Omega(\underline{u})), \\
\frac{\partial \underline{u}}{\partial n} & =c_{0} \text { on } \Gamma(\underline{u}) \text { in the sense of Definition 3.3, } \\
\lim _{y_{0} \rightarrow-\infty} \sup _{\Omega^{-}\left(y_{0}\right)} \underline{u} & =0, \\
\limsup _{d(X, \Gamma(\underline{u})) \rightarrow+\infty, X \in \Omega(\underline{u})} \underline{u} & =0
\end{aligned}\right.
$$

In particular, we see that $u$ is a solution of (3.2) if and only if it is both a sub- and a supersolution.

Remark 4.1 We could have defined weaker notions of solution, subsolution or supersolution of (3.2) by assuming less regularity for the Neumann conditions on the boundary $\Gamma$ (see Caffarelli [18], Ton $[49,50])$, but the uniformity of the behaviour of the functions near the free boundary will be used in the proofs, especially in the following proposition.

A nice property of the solutions (resp. supersolutions, subsolutions) of problem of the type (3.2) is the following compactness result.

Proposition 4.2 Let $\phi: \mathbb{R} \mapsto \mathbb{R}$ be a Lipschitz-continuous function and let $c_{1}, c_{2} \in \mathbb{R}$. Let $u$ (resp. $\bar{u}, \underline{u}$ ) be a globally Lipschitz-continuous solution (resp. supersolution, subsolution) of problem (3.2) such that $0<u \leqslant 1(\operatorname{resp} .0<\bar{u} \leqslant 1,0<\underline{u} \leqslant 1)$ in $\mathbb{R}^{2}$. Assume that $\Gamma(u)(\operatorname{resp} . \Gamma(\bar{u}), \Gamma(\underline{u}))$ is regular in the sense of Definition 3.1. Let $\left(x_{k}\right)_{k \in \mathbb{N}}$ be a sequence of real numbers and define

$$
u_{k}(x, y)=u\left(x_{k}+x, \phi\left(x_{k}\right)+y\right)
$$

$\left(\right.$ resp. $\left.\bar{u}_{k}(x, y)=\bar{u}\left(x_{k}+x, \phi\left(x_{k}\right)+y\right), \underline{u}_{k}(x, y)=\underline{u}\left(x_{k}+x, \phi\left(x_{k}\right)+y\right)\right)$ and $\phi_{k}(x)=\phi\left(x_{k}+\right.$ $x)-\phi\left(x_{k}\right)$.

Then, up to extraction of some subsequence, the functions $\phi_{k}$ converge uniformly locally in $\mathbb{R}$ to a function $\phi_{\infty}$ and the functions $u_{k}$ (resp. $\bar{u}_{k}, \underline{u}_{k}$ ) converge uniformly locally in $\mathbb{R}^{2}$ to $u_{\infty}$ (resp. $\bar{u}_{\infty}, \underline{u}_{\infty}$ ). Furthermore, $u_{\infty}$ (resp. $\bar{u}_{\infty}, \underline{u}_{\infty}$ ) is a solution (resp. supersolution, subsolution) of (3.2) with $\phi_{\infty}$ in place of $\phi$, namely

$$
\left\{\begin{aligned}
L u_{\infty} & =0 \text { in } \Omega\left(u_{\infty}\right), \\
\frac{\partial u_{\infty}}{\partial n} & =c_{0} \text { on } \Gamma\left(u_{\infty}\right) \text { in the sense of Definition 3.3 } \\
\lim _{y_{0} \rightarrow-\infty} \sup _{\Omega_{\infty}^{-}\left(y_{0}\right)} u_{\infty} & =0 \\
u_{\infty} & =1 \text { in } \Omega_{\infty}^{+}\left(y_{1}\right), \text { with the same } y_{1} \text { as in }(3.2)
\end{aligned}\right.
$$


resp. either $d\left(\left(x_{k}, \phi\left(x_{k}\right)\right), \Gamma(\bar{u})\right) \rightarrow+\infty$ and $\bar{u}_{\infty} \equiv 1$ in $\mathbb{R}^{2}$, or $d\left(\left(x_{k}, \phi\left(x_{k}\right)\right), \Gamma(\bar{u})\right)$ is bounded, both $\Omega\left(\bar{u}_{\infty}\right)$ and $\mathbb{R}^{2} \backslash \Omega\left(\bar{u}_{\infty}\right)$ are not empty and

$$
\left\{\begin{array}{l}
L \bar{u}_{\infty} \leqslant 0 \text { in } \mathcal{D}^{\prime}\left(\Omega\left(\bar{u}_{\infty}\right)\right), \\
\frac{\partial \bar{u}_{\infty}}{\partial n}=c_{0} \text { on } \Gamma\left(\bar{u}_{\infty}\right) \text { in the sense of Definition 3.3 } \\
\bar{u}_{\infty}=1 \text { in } \Omega_{\infty}^{+}\left(y_{1}\right), \text { with the same } y_{1} \text { as in (4.1), }
\end{array}\right.
$$

and resp. either $d\left(\left(x_{k}, \phi\left(x_{k}\right)\right), \Gamma(\underline{u})\right) \rightarrow+\infty$ and $\underline{u}_{\infty} \equiv 0$ in $\mathbb{R}^{2}$, or $d\left(\left(x_{k}, \phi\left(x_{k}\right)\right), \Gamma(\underline{u})\right)$ is bounded, both $\Omega\left(\underline{u}_{\infty}\right)$ and $\mathbb{R}^{2} \backslash \Omega\left(\underline{u}_{\infty}\right)$ are not empty and

$$
\left\{\begin{aligned}
L \underline{u}_{\infty} & \geqslant 0 \text { in } \mathcal{D}^{\prime}\left(\Omega\left(\underline{u}_{\infty}\right)\right), \\
\frac{\partial \underline{u}_{\infty}}{\partial n} & =c_{0} \text { on } \Gamma\left(\underline{u}_{\infty}\right) \text { in the sense of Definition 3.3 } \\
\lim _{y_{0} \rightarrow-\infty} \sup _{\Omega_{\infty}^{-}\left(y_{0}\right)} \underline{u}_{\infty} & =0 \\
\lim _{\substack{\operatorname{lop} \\
d\left(\Gamma\left(\underline{u}_{\infty}\right)\right) \rightarrow+\infty \\
X \in \Omega\left(\underline{u}_{\infty}\right)}} \underline{u}_{\infty} & =0
\end{aligned}\right.
$$

where

$$
\forall y_{0} \in \mathbb{R},\left\{\begin{array}{l}
\Omega_{\infty}^{-}\left(y_{0}\right)=\left\{y<\phi_{\infty}(x)+y_{0}\right\}, \\
\Omega_{\infty}^{+}\left(y_{0}\right)=\left\{y>\phi_{\infty}(x)+y_{0}\right\} .
\end{array}\right.
$$

Moreover, $\Gamma\left(u_{\infty}\right)\left(\operatorname{resp} . \Gamma\left(\bar{u}_{\infty}\right), \Gamma\left(\underline{u}_{\infty}\right)\right)$ is regular in the sense of Definition 3.1 and

$$
\lim _{k \rightarrow+\infty} \Omega\left(u_{k}\right)=\overline{\Omega\left(u_{\infty}\right)}
$$

(resp. $\left.\lim _{k \rightarrow+\infty} \Omega\left(\bar{u}_{k}\right)=\overline{\Omega\left(\bar{u}_{\infty}\right)}, \lim _{k \rightarrow+\infty} \Omega\left(\underline{u}_{k}\right)=\overline{\Omega\left(\underline{u}_{\infty}\right)}\right)$, where for any sequence of sets $E_{k}$, $\lim _{k \rightarrow+\infty} E_{k}:=\left\{X \in \mathbb{R}^{2}, \exists X_{k} \in E_{k}, X=\lim _{k \rightarrow+\infty} X_{k}\right\}$.

Proof. This is done only in the case where $u$ is a solution of (3.2). The proofs in the cases of suband supersolutions are similar and use some parts of the proof below (see Remark 4.3).

Let then $u$ be a globally Lipschitz-continuous solution of (3.2). For the sake of clarity, one divides the proof into four main steps.

Step 1: existence of $u_{\infty}$ and $\phi_{\infty}$. Under the assumption of Proposition 4.2, the family of functions $\left(u_{k}\right)$ is equi-Lipschitz-continuous and globally bounded between 0 and 1 . From Ascoli's theorem, the functions $u_{k}$ converge locally uniformly in $\mathbb{R}^{2}$, up to extraction of some subsequence, to a globally Lipschitz-continuous function $u_{\infty}$ such that $0 \leqslant u_{\infty} \leqslant 1$ in $\mathbb{R}^{2}$. Furthermore,

$$
L u_{\infty}=0 \text { in } \mathcal{D}^{\prime}\left(\Omega\left(u_{\infty}\right)\right) \text { where } \Omega\left(u_{\infty}\right)=\left\{0 \leqslant u_{\infty}<1\right\} .
$$

From standard elliptic estimates, $L u_{\infty}=0$ actually holds in the classical sense in $\Omega\left(u_{\infty}\right)$.

Similarly, the functions $\phi_{k}$ converge, up to extraction of some subsequence, locally uniformly in $\mathbb{R}$ to a globally Lipschitz continuous function $\phi_{\infty}$.

The limiting condition

$$
\lim _{y_{0} \rightarrow-\infty} \sup _{\Omega_{\infty}^{-}\left(y_{0}\right)} u_{\infty}=0
$$


follows from $\lim _{y_{0} \rightarrow-\infty} \sup _{\Omega^{-}\left(y_{0}\right)} u=0$ and from the definition of $\phi_{k}$. Indeed, take any $\varepsilon>0$ and let $y_{0}$ be such that $u(x, y) \leqslant \varepsilon$ for all $y<\phi(x)+y_{0}$. Now choose any point $(x, y)$ such that $y<\phi_{\infty}(x)+y_{0}$. For $k$ large enough, one then has $y<\phi_{k}(x)+y_{0}$, i.e. $y+\phi\left(x_{k}\right)<\phi\left(x_{k}+x\right)+y_{0}$. Therefore,

$$
u\left(x_{k}+x, y+\phi\left(x_{k}\right)\right) \leqslant \varepsilon .
$$

In other words, $u_{k}(x, y) \leqslant \varepsilon$ for $k$ large enough, whence $u_{\infty}(x, y) \leqslant \varepsilon$, which is what was to be proved.

Similarly, it is esay to check that $u_{\infty}=1$ in $\Omega_{\infty}^{+}\left(y_{1}\right)$ with the same $y_{1}$ as in (3.2).

Step 2: proof of the Neumann condition on $\Gamma\left(u_{\infty}\right)$. Let us now prove that the Neumann condition $\frac{\partial u_{\infty}}{\partial n}=c_{0}$ holds on $\Gamma\left(u_{\infty}\right)$ in the sense of Definition 3.3. First, since the function $u$ itself satisfies $\frac{\partial u}{\partial n}$ on $\Gamma(u)$, there exist two functions $h_{1}$ and $h_{2}$ fulfilling the requirements of Definition 3.3. To prove that $u_{\infty}$ also satisfies this Neumann condition on $\Gamma\left(u_{\infty}\right)$, it is sufficient to show that

$$
\forall X \in \Omega\left(u_{\infty}\right), \quad 1-h_{1}(r) \leqslant u_{\infty}(X) \leqslant 1-h_{2}(r), \text { where } r=d\left(X, \Gamma\left(u_{\infty}\right)\right) .
$$

To prove (4.6), choose an arbitrary point $X=(x, y) \in \Omega\left(u_{\infty}\right)$ (i.e. $\left.u_{\infty}(X)<1\right)$. For $k$ large enough, one has $u_{k}(X)<1$, whence

$$
1-h_{1}\left(r_{k}\right) \leqslant u\left(X_{k}\right) \leqslant 1-h_{2}\left(r_{k}\right)
$$

where $X_{k}=\left(x_{k}+x, \phi\left(x_{k}\right)+y\right)$ and $r_{k}=d\left(X_{k}, \Gamma(u)\right)$ (see Figure 2). On the other hand, one has $u\left(x_{k}, \phi\left(x_{k}\right)+y_{1}\right)=1$. Therefore, the segment $S$ between the points $X_{k}$ and $\left(x_{k}, \phi\left(x_{k}\right)+y_{1}\right)$ meets $\Gamma(u)$. As a consequence, $r_{k}$ is not larger than the length of $S$, which is independent of $k$. Finally, the sequence $\left(r_{k}\right)$ is bounded. Let now $Z_{k}=\left(x_{k}, \phi\left(x_{k}\right)\right)+Y_{k}$ be a point on $\Gamma(u)$ such that

$$
\left|X_{k}-Z_{k}\right|=\left|X-Y_{k}\right|=r_{k}
$$

(such a point exists because $\Gamma(u)$ is closed). Up to extraction of some subsequence, one has $Y_{k} \rightarrow$ $Y_{\infty} \in \mathbb{R}^{2}$ and $r_{k} \rightarrow r_{\infty}=\left|X-Y_{\infty}\right| \in[0,+\infty)$. Since the functions $u_{k}$ are equi-Lipschitzcontinuous and converge to $u_{\infty}$ and since $u_{k}\left(Y_{k}\right)=1$, it follows that $u_{\infty}\left(Y_{\infty}\right)=1$. Since $u_{\infty}(X)<$ 1 , one gets $r_{\infty}>0$.

Now choose any point $\tilde{X}=(\tilde{x}, \tilde{y})$ such that $|\tilde{X}-X|<r_{\infty}$ and set $\tilde{X}_{k}=\left(x_{k}+\tilde{x}, \phi\left(x_{k}\right)+\tilde{y}\right)$. For $k$ large enough, one has $|\tilde{X}-X|<r_{k}$. Since $\Gamma(u) \subset \mathbb{R}^{2} \backslash B\left(X_{k}, r_{k}\right)$ (where $B\left(X_{k}, r_{k}\right)$ denotes the open ball with centre $X_{k}$ and radius $r_{k}$ ) and since $r_{k}=d\left(X_{k}, \Gamma(u)\right)$, it follows that

$$
d\left(\tilde{X}_{k}, \Gamma(u)\right) \geqslant r_{k}-\left|\tilde{X}_{k}-X_{k}\right|=r_{k}-|\tilde{X}-X| .
$$

On the other hand, $d\left(\tilde{X}_{k}, \Gamma(u)\right) \leqslant d\left(\tilde{X}_{k}, Z_{k}\right) \leqslant 2 r_{k}$. Finally, (3.3) yields

$$
1-\sup _{\left[r_{k}-|\tilde{X}-X|,\left|\tilde{X}_{k}-Z_{k}\right|\right]} h_{1} \leqslant u\left(\tilde{X}_{k}\right)=u_{k}(\tilde{X}) \leqslant 1-\inf _{\left[r_{k}-|\tilde{X}-X|,\left|\tilde{X}_{k}-Z_{k}\right|\right]} h_{2} .
$$

For $k$ large enough, one has

$$
r_{k}-|\tilde{X}-X| \geqslant\left(r_{\infty}-|\tilde{X}-X|\right) / 2>0
$$

and, since $\left|\tilde{X}_{k}-Z_{k}\right|$ is bounded and $h_{2}$ is continuous and positive on $(0,+\infty)$, the passage to the limit $k \rightarrow+\infty$ leads to $u_{\infty}(\tilde{X})<1$. As a consequence, $B\left(X, r_{\infty}\right) \subset \Omega\left(u_{\infty}\right)$. But $u_{\infty}\left(Y_{\infty}\right)=1$ and $\left|Y_{\infty}-X\right|=r_{\infty}$. Therefore, it follows that $r_{\infty}=d\left(X, \Gamma\left(u_{\infty}\right)\right)$. 


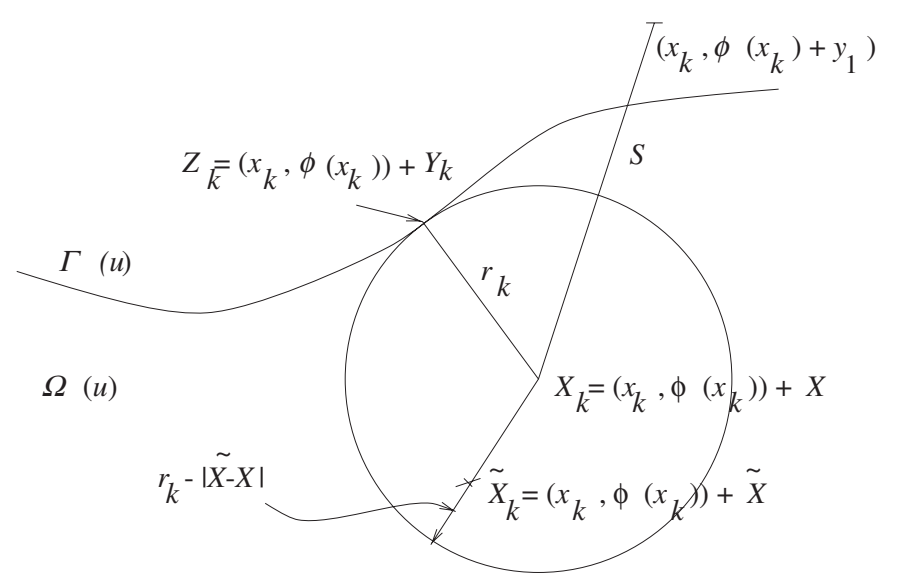

FIG. 2. The ball of radius $r_{k}$ and centre $X_{k}$.

Applying (4.7) to $\tilde{X}=X$ leads to

$$
1-h_{1}\left(r_{k}\right) \leqslant u_{k}(X) \leqslant 1-h_{2}\left(r_{k}\right)
$$

and the limit $k \rightarrow+\infty$ yields (4.6). That means that $u_{\infty}$ satisfies the Neumann condition $\frac{\partial u_{\infty}}{\partial n}=c_{0}$ on $\Gamma\left(u_{\infty}\right)$ in the sense of Definition 3.3.

Step 3: convergence of $\Omega\left(u_{k}\right)$ to $\overline{\Omega\left(u_{\infty}\right)}$. First of all, any point $X \in \Omega\left(u_{\infty}\right)$ is such that $X \in$ $\Omega\left(u_{k}\right)$ for $k$ large enough. Next, let us prove that any point on $\Gamma\left(u_{\infty}\right)=\partial \Omega\left(u_{\infty}\right)$ is the limit of a sequence of points in $\Omega\left(u_{k}\right)$. Choose any $Y_{\infty} \in \Gamma\left(u_{\infty}\right)$. Let us first prove the existence of a sequence of points $Y_{k} \in \Gamma\left(u_{k}\right)$ such that $Y_{k} \rightarrow Y_{\infty}$ as $k \rightarrow+\infty$. Indeed, if not, there exists an open ball $B_{r}\left(Y_{\infty}\right)$ centred at $Y_{\infty}$, with positive radius $r$, such that, for a sequence $k \rightarrow+\infty$,

$$
\text { either } B_{r}\left(Y_{\infty}\right) \subset\left\{u_{k}=1\right\}^{\mathrm{O}} \text { or } B_{r}\left(Y_{\infty}\right) \subset\left\{u_{k}<1\right\} .
$$

The first case is clearly impossible, otherwise $u_{\infty}=1$ in $B_{r}\left(Y_{\infty}\right)$ and $Y_{\infty}$ cannot be on $\Gamma\left(u_{\infty}\right)$. If the second case occurs, call $r_{k}=d\left(Y_{\infty}, \Gamma\left(u_{k}\right)\right) \geqslant r$. As in step 2, the sequence $\left(r_{k}\right)$ is bounded and, up to extraction of some subsequence, $r_{k} \rightarrow r_{\infty} \geqslant r$ and

$$
u_{\infty}\left(Y_{\infty}\right) \leqslant 1-h_{2}\left(r_{\infty}\right)<1 .
$$

The latter is in contradiction with $Y_{\infty} \in \Gamma\left(u_{\infty}\right)$ and this second case is ruled out too. Therefore, $\overline{\Omega\left(u_{\infty}\right)} \subset \lim _{k \rightarrow+\infty} \Omega\left(u_{k}\right)$.

Let us now prove the reverse inclusion. Take a sequence $X_{k} \in \Omega\left(u_{k}\right)$ such that $X_{k} \rightarrow X$ as $k \rightarrow+\infty$. Let $r_{k}=d\left(X_{k}, \Gamma\left(u_{k}\right)\right)$. As in step 2, the sequence $\left(r_{k}\right)$ is bounded and, up to extraction of some subsequence, one has either $r_{k} \rightarrow r_{\infty}>0$ or $r_{k} \rightarrow 0$. In the first case, it follows as in step 2 that

$$
u_{\infty}(X) \leqslant 1-h_{2}\left(r_{\infty}\right)<1 \text {, }
$$

whence $X \in \Omega\left(u_{\infty}\right)$. If the second case occurs, there then exists a sequence of points $Y_{k} \in \Gamma\left(u_{k}\right)$ such that $\left|X_{k}-Y_{k}\right|=r_{k} \rightarrow 0$, whence $Y_{k} \rightarrow X$. Since $\Gamma(u)$ is regular in the sense of Definition 3.1 and since each $\Gamma\left(u_{k}\right)$ is just a translation of $\Gamma(u)$, there exists for each $k$ a ball $B_{k}^{-} \subset \Omega\left(u_{k}\right)$ of 
radius $\delta$ and centre $W_{k}$, and such that $Y_{k} \in \partial B_{k}^{-}$. Since $\left|Y_{k}-W_{k}\right|=\delta$ and $Y_{k} \rightarrow X$, one can assume, up to extraction of some subsequence, that $W_{k} \rightarrow W$ with $|X-W|=\delta$. As in step 2, it follows that the open ball $B_{\delta}(W)$ is included in $\Omega\left(u_{\infty}\right)$. Since $|X-W|=\delta$, one concludes that $X \in \overline{\Omega\left(u_{\infty}\right)}$.

Eventually, $\lim _{k \rightarrow+\infty} \Omega\left(u_{k}\right)=\overline{\Omega\left(u_{\infty}\right)}$.

Step 4: the free boundary $\Gamma\left(u_{\infty}\right)$ is regular. Choose any $Y_{\infty} \in \Gamma\left(u_{\infty}\right)$. From step 3, there exists a sequence of points $Y_{k} \in \Gamma\left(u_{k}\right)$ such that $Y_{k} \rightarrow Y_{\infty}$ as $k \rightarrow+\infty$. Since the boundary $\Gamma(u)$ is regular in the sense of Definition 3.1, there exist then $\delta>0$ and two sequences of points $X_{k}^{ \pm}$such that

$$
B_{\delta}\left(X_{k}^{-}\right) \subset \Omega\left(u_{k}\right), \quad \partial B_{\delta}\left(X_{k}^{-}\right) \cap \partial B_{\delta}\left(X_{k}^{+}\right)=\left\{Y_{k}\right\} \text { and } B_{\delta}\left(X_{k}^{+}\right) \cap \mathcal{C}_{k}^{-}=\emptyset,
$$

where $\mathcal{C}_{k}^{-}$is the connected component of $\Omega\left(u_{k}\right) \cap B_{2 \delta}\left(Y_{k}\right)$ which contains $B_{\delta}\left(X_{k}^{-}\right)$. Up to extraction of some subsequence, one has $X_{k}^{ \pm} \rightarrow X_{\infty}^{ \pm}$together with $\partial B_{\delta}\left(X_{\infty}^{-}\right) \cap \partial B_{\delta}\left(X_{\infty}^{+}\right)=\left\{Y_{\infty}\right\}$. The same arguments as in step 2 show that $B_{\delta}\left(X_{\infty}^{-}\right) \subset \Omega\left(u_{\infty}\right)$.

On the other hand, let $v_{k}$ be the function defined in $B_{2 \delta}\left(Y_{k}\right)$ by

$$
v_{k}(X)= \begin{cases}u_{k}(X) & \text { in } \mathcal{C}_{k}^{-}, \\ 1 & \text { in } B_{2 \delta}\left(Y_{k}\right) \backslash \mathcal{C}_{k}^{-}\end{cases}
$$

These functions are equi-Lipschitz-continuous in $B_{2 \delta}\left(Y_{k}\right)$ and, up to extraction of some subsequence, they converge locally in $B_{2 \delta}\left(Y_{\infty}\right)$ to a Lipschitz-continuous function $v_{\infty}$. Call $E=$ $\lim _{k \rightarrow+\infty} \mathcal{C}_{k}^{-}$. By definition of $\mathcal{C}_{k}^{-}$, the set $E$ contains $B_{\delta}\left(X_{\infty}^{-}\right)$. One also has $v_{\infty}=u_{\infty}$ in $E$ and, from the arguments of steps 2 and 3 , it follows that

$$
v_{\infty}=u_{\infty}=1 \text { in } \partial E \cap B_{2 \delta}\left(Y_{\infty}\right) .
$$

Furthermore, $\mathcal{C}_{k}^{-} \cap B_{\delta}\left(X_{k}^{+}\right)=\emptyset$ implies that $E \cap B_{\delta}\left(X_{\infty}^{+}\right)=\emptyset$. Eventually, the connected component of $\Omega\left(u_{\infty}\right) \cap B_{2 \delta}\left(Y_{\infty}\right)$ containing $B_{\delta}\left(X_{\infty}^{-}\right)$does not meet $B_{\delta}\left(X_{\infty}^{+}\right)$.

Since $\delta$ is independent of $Y_{\infty} \in \Gamma\left(u_{\infty}\right)$, that means that $\Gamma\left(u_{\infty}\right)$ is regular in the sense of Definition 3.1 and the proof of Proposition 4.2 is complete.

Remark 4.3 In the case of solution $u$, the arguments in step 1 yield that $\Gamma\left(u_{\infty}\right) \neq \emptyset$.

In the case of subsolution $\underline{u}$, two cases may occur: up to extraction of some subsequence, either $d\left(\left(x_{k}, \phi\left(x_{k}\right)\right), \Gamma(\underline{u})\right)$ is bounded, or $d\left(\left(x_{k}, \phi\left(x_{k}\right)\right), \Gamma(\underline{u})\right) \rightarrow+\infty$. If the first case occurs, the same arguments as used in steps 1-4 guarantee that both $\Omega\left(\underline{u}_{\infty}\right)$ and $\mathbb{R}^{2} \backslash \Omega\left(\bar{u}_{\infty}\right)$ are not empty, whence $\Gamma\left(\underline{u}_{\infty}\right) \neq \emptyset$, and that, under the same notations as above, all sequences $\left(r_{k}\right)$ that have been used remain bounded. If $d\left(\left(x_{k}, \phi\left(x_{k}\right)\right), \Gamma(\underline{u})\right) \rightarrow+\infty$, then it is easy to see that $\left(x_{k}, \phi\left(x_{k}\right)\right) \in \Omega(\underline{u})$ for $k$ large enough and that $\underline{u}_{\infty} \equiv 0$ in $\mathbb{R}^{2}$.

In the case of supersolution $\bar{u}$, it also follows easily that if $d\left(\left(x_{k}, \phi\left(x_{k}\right)\right), \Gamma(\bar{u})\right) \rightarrow+\infty$, then $\bar{u}_{\infty} \equiv 1$ and $\Omega\left(\bar{u}_{\infty}\right)=\Gamma\left(\bar{u}_{\infty}\right)=\emptyset$, while if $d\left(\left(x_{k}, \phi\left(x_{k}\right)\right), \Gamma(\bar{u})\right)$ is bounded then both $\Omega\left(\bar{u}_{\infty}\right)$ and $\Gamma\left(\bar{u}_{\infty}\right)$ are not empty.

Remark 4.4 If $u$ is a solution of (3.2) such that $\Omega(u)=\{y<\phi(x)\}$, then we can check immediately that, using the notation of Proposition 4.2,

$$
\Omega\left(u_{\infty}\right)=\left\{y<\phi_{\infty}(x)\right\} .
$$

Let us now state one of the main results in this paper, namely a comparison principle between super- and subsolutions of problems of type (3.2). This result, which we will make constant use of 
in the course of the proof of Theorem 1.6, says especially that given a Lipschitz-continuous function $\phi$, a supersolution $\bar{u}$ and a subsolution $\underline{u}$ of problem (3.2) in the sense of (4.1)-(4.2), then $\bar{u}$ can be slid in the $y$-direction in order to be above $\underline{u}$ everywhere in $\mathbb{R}^{2}$.

In what follows, for any function $w: \overline{\mathbb{R}^{2}} \rightarrow \mathbb{R}$ and any $t \in \mathbb{R}, w^{t}$ denotes the function defined by $w^{t}(x, y)=w(x, y+t)$.

Theorem 4.5 (Comparison principle) Let $\phi: \mathbb{R} \rightarrow \mathbb{R}$ be a given Lipschitz-continuous function. Let $\bar{u}$ (resp. $\underline{u}$ ) be a Lipschitz supersolution of (4.1) (resp. Lipschitz subsolution of (4.2)) such that $0 \leqslant \bar{u} \leqslant 1$ (resp. $0 \leqslant \underline{u} \leqslant 1)$ in $\mathbb{R}^{2}$ and assume that $\Gamma(\bar{u})($ resp. $\Gamma(\underline{u})$ ) is regular in the sense of Definition 3.1. Then the set

$$
I=\left\{t \in \mathbb{R}, \forall s \geqslant t, \bar{u}^{s} \geqslant \underline{u}\right\}
$$

is not empty. Furthermore, if $t^{*}:=\inf I>-\infty$, then $\bar{u}^{t^{*}} \geqslant \underline{u}$ and

(i) if $\Gamma\left(\bar{u}^{t^{*}}\right) \cap \Gamma(\underline{u}) \neq \emptyset$, then $\bar{u}^{t^{*}}=\underline{u}$ in $\Omega\left(\bar{u}^{t^{*}}\right)$,

(ii) if $\Gamma\left(\bar{u}^{t^{*}}\right) \cap \Gamma(\underline{u})=\emptyset$, then $\bar{u}^{t^{*}}>\underline{u}$ in $\Omega(\underline{u})$ and there exists a sequence $\left|x_{k}\right| \rightarrow+\infty$ such that the sequences $d\left(\left(x_{k}, \phi\left(x_{k}\right)\right), \Gamma(\bar{u})\right)$ and $d\left(\left(x_{k}, \phi\left(x_{k}\right)\right), \Gamma(\underline{u})\right)$ are bounded and, under the notation of Proposition 4.2, the functions $\bar{u}_{k}^{t^{*}}, \underline{u}_{k}$ converge to some functions $\bar{u}_{\infty}^{t^{*}}, \underline{u}_{\infty}$, which are not identically equal to 1 and 0 respectively and which satisfy $\bar{u}_{\infty}^{t^{*}}=\underline{u}_{\infty}$ in $\Omega\left(\bar{u}_{\infty}^{t^{*}}\right)$.

Moreover, if $\bar{u}$ and $\underline{u}$ are nondecreasing with respect to the variable $y$, then $\bar{u}^{t^{*}} \equiv \underline{u}$ in $\mathbb{R}^{2}$ in case (i) and $\bar{u}_{\infty}^{t^{*}} \equiv \underline{u}_{\infty}$ in $\mathbb{R}^{2}$ in case (ii).

Remark 4.6 Similar results hold in $\mathbb{R}^{N}$ for any dimension $N$.

Postponing the proof of Theorem 4.5 in Section 6, let us now turn to the proof of Theorem 3.4.

Proof of Theorem 3.4. Apply Theorem 4.5 with $\bar{u}=\underline{u}=u$. The set $I=\left\{t \in \mathbb{R}, \forall s \geqslant t, u^{s} \geqslant u\right.$ in $\left.\mathbb{R}^{2}\right\}$ is not empty. Furthermore, $t^{*}=\inf I>-\infty$ because on the one hand there exists $y_{1} \in \mathbb{R}$ such that $u=1$ in $\Omega^{+}\left(y_{1}\right)$ and on the other hand

$$
\lim _{y_{0} \rightarrow-\infty} \sup _{\Omega^{-}\left(y_{0}\right)} u=0
$$

In order to prove that $u$ is nondecreasing with respect to the variable $y$, it is sufficient to show that $t^{*} \leqslant 0$. Assume on the contrary that $t^{*}>0$. Under the notation of Theorem 4.5, if case (i) occurs, then $u^{t^{*}}=u$ in $\Omega\left(u^{t^{*}}\right)=\Omega(u)-\left(0, t^{*}\right)$. Take a point $(x, y) \in \Omega\left(u^{t^{*}}\right)$. Since $u\left(x, y+t^{*}\right)=u(x, y)$, it follows that $(x, y) \in \Omega(u)$, i.e. $\left(x, y-t^{*}\right) \in \Omega\left(u^{t^{*}}\right)$. As a consequence, $u(x, y)=u\left(x, y-t^{*}\right)$. An immediate induction yields

$$
u(x, y)=u\left(x, y-n t^{*}\right) \text { for all } n \in \mathbb{N} .
$$

The limit $n \rightarrow+\infty$ gives $u(x, y)=0$ thanks to (4.8). This is impossible since $u$ is assumed to be positive in $\mathbb{R}^{2}$.

Therefore, under the notation of Theorem 4.5, case (ii) occurs and $u_{\infty}^{t^{*}}=u_{\infty}$ in $\Omega\left(u_{\infty}^{t^{*}}\right)$. From Proposition 4.2, the function $u_{\infty}$ is Lipschitz-continuous and solves (4.3). The strong maximum principle implies that $u_{\infty}$ is positive in $\mathbb{R}^{2}$. One is then led to a contradiction as in case (i).

Consequently, $t^{*} \leqslant 0$ (one could also prove with similar arguments that $t^{*} \geqslant 0$ ) and the proof of Theorem 3.4 is complete. 


\section{Proof of the Serrin-type result (Theorem 1.6)}

This section is devoted to the proof of Theorem 1.6. We repeat that this theorem includes the result of Theorem 1.3. We shall here use the comparison principle and the monotonicity results stated in the previous sections. For the sake of clarity, the proof of Theorem 1.6 is divided into several steps.

Let $(c, u, \Omega)$ be a solution of (1.1), where $\Omega$ is open, both $\Omega$ and $\mathbb{R}^{2} \backslash \Omega$ are not empty, the free boundary $\Gamma=\partial \Omega$ is globally $C^{1,1}$ with bounded curvature and the restriction $\tilde{u}$ of $u$ in $\bar{\Omega}$ is $C^{1}$ up to $\Gamma$. We assume moreover that $\mathbb{R}^{2} \backslash \Omega$ has no bounded connected components.

First of all, it follows from standard elliptic estimates up to the boundary [40] that $\tilde{u}$ is globally $C^{1, \beta}(\bar{\Omega})$ for each $\beta \in[0,1)$. Hence, the function $u$ is globally Lipschitz-continuous in $\mathbb{R}^{2}$. Next, Propositions 3.7 and 3.9 imply that, up to changing $(c, u, \Omega)$ into $(-c, u(-x,-y),-\Omega)$, the real $c$ is non-negative,

$$
\begin{aligned}
& \exists \phi: \mathbb{R} \rightarrow \mathbb{R} \text { globally Lipschitz-continuous such that } \\
& \Omega=\{y<\phi(x)\} \text { and } \Gamma=\{y=\phi(x)\}
\end{aligned}
$$

and the function $u$ is a solution of (3.2) in the sense of the Definitions 3.1 and 3.3, with $\left(c_{1}, c_{2}\right)=$ $(0,-c)$

Let $\alpha \in(0, \pi / 2]$ be the unique angle such that

$$
\cot \alpha=\operatorname{Lip}(\phi):=\sup _{\mathrm{x} \neq \mathrm{x}^{\prime}} \frac{\left|\phi(\mathrm{x})-\phi\left(\mathrm{x}^{\prime}\right)\right|}{\left|\mathrm{x}-\mathrm{x}^{\prime}\right|} .
$$

From Corollary 3.5, one immediately has the following corollary.

Corollary 5.1 (i) The function $u$ is nonincreasing in all the directions $\tau$ such that $\|\tau\|=1$ and $\tau_{y} \leqslant-\cos \alpha$.

(ii) $\left|\phi(x)-\phi\left(x^{\prime}\right)\right| \leqslant \cot \alpha\left|x-x^{\prime}\right|$ for all $x, x^{\prime} \in \mathbb{R}$.

(iii) For any point $(x, y) \in \Gamma, \mathcal{C}(x, y) \subset \Omega$ where

$$
\mathcal{C}(x, y)=\{(x, y)+\lambda(\cos \varphi, \sin \varphi), \lambda>0,-\pi / 2-\alpha<\varphi<-\pi / 2+\alpha\} .
$$

Step 1: proof of the formula $c=c_{0} / \sin \alpha$. For each $\lambda>0$, let $\alpha_{\lambda} \in(0, \pi / 2]$ be defined by

$$
\cot \alpha_{\lambda}:=\sup _{p \in \mathbb{Z}} \frac{|\phi((p+1) \lambda)-\phi(p \lambda)|}{\lambda} .
$$

Note that $\cot \alpha_{\lambda} \leqslant \operatorname{Lip}(\phi)$ for all $\lambda>0$.

We then have the following lemma.

Lemma 5.2 For all $\lambda>0, \cot \alpha_{\lambda}=\operatorname{Lip}(\phi)$.

Proof. For each $\lambda>0$, let $\psi_{\lambda}: \mathbb{R} \rightarrow \mathbb{R}$ be the Lipschitz-continuous function whose graph links the points $(p \lambda, \phi(p \lambda))_{p \in \mathbb{Z}}$. Then $\operatorname{Lip}\left(\psi_{\lambda}\right)=\cot \alpha_{\lambda}$ and we can immediately see that there exist $a, b \in \mathbb{R}$ such that

$$
\forall x \in \mathbb{R}, \quad \psi_{\lambda}(x)+a \leqslant \phi(x) \leqslant \psi_{\lambda}(x)+b .
$$

Consequently $u$ is a solution of (3.2) with $\psi_{\lambda}$ in place of $\phi$ in the definition of (3.2). Thus Corollary 3.5 can be applied and proves that the function $u$ is nonincreasing in all directions of 
the cone $\mathcal{C}^{-}\left(\alpha_{\lambda}\right)$. Now assume that $\operatorname{Lip}\left(\psi_{\lambda}\right)<\operatorname{Lip}(\phi)$ and take two real numbers $x, x^{\prime} \in \mathbb{R}$ such that $x<x^{\prime}$ and

$$
\cot \alpha_{\lambda}=\operatorname{Lip}\left(\psi_{\lambda}\right)<\frac{\left|\phi(\mathrm{x})-\phi\left(\mathrm{x}^{\prime}\right)\right|}{\left|\mathrm{x}-\mathrm{x}^{\prime}\right|} \leqslant \operatorname{Lip}(\phi)
$$

Suppose that $\phi(x)>\phi\left(x^{\prime}\right)$ (the case $\phi(x)<\phi\left(x^{\prime}\right)$ can be treated similarly). Therefore,

$$
\left(x^{\prime}-x\right) \cot \alpha_{\lambda}<\phi(x)-\phi\left(x^{\prime}\right) \leqslant \operatorname{Lip}(\phi)\left(\mathrm{x}^{\prime}-\mathrm{x}\right) \text {. }
$$

From Corollary 5.1, one has $u(x, \phi(x)-\varepsilon)<1$ for all $\varepsilon>0$. Since $u$ is nonincreasing in all directions of the cone $\mathcal{C}^{-}\left(\alpha_{\lambda}\right)$, it follows that

$$
\forall \varepsilon>0, \quad u\left(x^{\prime}, \phi(x)-\varepsilon-\left(x^{\prime}-x\right) \cot \alpha_{\lambda}\right) \leqslant u(x, \phi(x)-\varepsilon)<1 .
$$

Choosing $\varepsilon=\phi(x)-\phi\left(x^{\prime}\right)-\left(x^{\prime}-x\right) \cot \alpha_{\lambda}>0$ yields $u\left(x^{\prime}, \phi\left(x^{\prime}\right)\right)<1$, which is impossible.

Therefore, $\operatorname{Lip}\left(\psi_{\lambda}\right) \geqslant \operatorname{Lip}(\phi)$ and formula (5.2) leads to $\cot \alpha_{\lambda}=\operatorname{Lip}(\phi)$. That completes the proof of Lemma 5.2.

From Lemma 5.2, for each $\lambda>0$, there exists a sequence of integers $\left(p_{k}\right)_{k}$ such that

$$
\frac{\left|\phi\left(\left(p_{k}+1\right) \lambda\right)-\phi\left(p_{k} \lambda\right)\right|}{\lambda} \longrightarrow \operatorname{Lip}(\phi)=\cot \alpha \text { as } \mathrm{k} \rightarrow+\infty .
$$

Let $x_{k}=\left(p_{k}+1 / 2\right) \lambda, \phi_{k}(x)=\phi\left(x_{k}+x\right)-\phi\left(x_{k}\right)$ and $u_{k}(x, y)=u\left(x_{k}+x, \phi\left(x_{k}\right)+y\right)$. From Proposition 4.2 and Remark 4.4, it follows that, up to extraction of some subsequence, the functions $\phi_{k}(x)$ and $u_{k}(x, y)$ converge locally in $\mathbb{R}$ and $\mathbb{R}^{2}$ respectively to two globally Lipschitz-continuous functions $\phi_{\infty}^{\lambda}(x)$ and $u_{\infty}^{\lambda}(x, y)$ solving

$$
\left\{\begin{array}{rlrl}
\Delta u_{\infty}^{\lambda}-c \partial_{y} u_{\infty}^{\lambda} & =0 \text { in } & \Omega_{\infty}^{\lambda}:=\left\{u_{\infty}^{\lambda}<1\right\}=\left\{y<\phi_{\infty}^{\lambda}(x)\right\}, \\
u_{\infty}^{\lambda}=1 \text { in } & \mathbb{R}^{2} \backslash \Omega_{\infty}^{\lambda}, \\
\frac{\partial u_{\infty}^{\lambda}}{\partial n} & =c_{0} \text { on } & & \Gamma\left(u_{\infty}^{\lambda}\right):=\partial \Omega_{\infty}^{\lambda}=\left\{y=\phi_{\infty}^{\lambda}(x)\right\}, \\
\lim _{y_{0} \rightarrow-\infty} \sup _{y<y_{0}+\phi_{\infty}^{\lambda}(x)} u_{\infty}^{\lambda}(x, y) & =0 & &
\end{array}\right.
$$

in the sense of Definitions 3.1 and 3.3. Moreover

$$
\frac{\left|\phi_{\infty}^{\lambda}(\lambda / 2)-\phi_{\infty}^{\lambda}(-\lambda / 2)\right|}{\lambda}=\cot \alpha
$$

and $\operatorname{Lip}\left(\phi_{\infty}^{\lambda}\right) \leqslant \cot \alpha$. Then, there exists $\varepsilon_{\lambda}= \pm 1$ such that $\phi_{\infty}^{\lambda}(x)=\varepsilon_{\lambda} x \cot \alpha$ for all $x \in$ $[-\lambda / 2, \lambda / 2]$.

We then pass to the limit for a sequence $\lambda_{n} \rightarrow+\infty$. Up to extraction of some subsequence, the functions $\phi_{\infty}^{\lambda_{n}}(x)$ and $u_{\infty}^{\lambda_{n}}(x, y)$ converge locally in $\mathbb{R}$ and $\mathbb{R}^{2}$ respectively to two functions $\phi_{\infty \infty}(x)$ and $u_{\infty \infty}(x, y)$, where $u_{\infty \infty}$ satisfies (5.3) with $\phi_{\infty \infty}$ in place of $\phi_{\infty}^{\lambda}$. Furthermore, there exists $\varepsilon= \pm 1$ such that

$$
\phi_{\infty \infty}(x)=\varepsilon x \cot \alpha \quad \text { for all } x \in \mathbb{R} .
$$

Let us assume that $\phi_{\infty \infty}(x)=x \cot \alpha$ for all $x \in \mathbb{R}$ (the case $\phi_{\infty \infty}(x)=-x \cot \alpha$ can be treated similarly). In the new coordinates $(X, Y)=(x \cos \alpha-y \sin \alpha, x \sin \alpha+y \cos \alpha)$, the function 
$v(X, Y)=u_{\infty \infty}(x, y)$ satisfies

$$
\left\{\begin{aligned}
\Delta v+c \sin \alpha \partial_{X} v-c \cos \alpha \partial_{Y} v & =0 \text { in } & & \{v<1\}=\{X>0\}, \\
v & =1 \text { in } & & \{X \leqslant 0\}, \\
\frac{\partial v}{\partial n} & =c_{0} \text { on } & & \{X=0\}, \\
\lim _{X_{0} \rightarrow+\infty} \sup _{X>X_{0}, Y \in \mathbb{R}} v(X, Y) & =0, & &
\end{aligned}\right.
$$

in the sense of Definitions 3.1 and 3.3. By rotating the $(X, Y)$ frame clockwise or counterclockwise by any angle less than $\pi / 2$ and by applying Theorem 3.4, it follows that the function $v$ is nonincreasing in any direction $\tau$ such that $\tau_{X}>0$. By continuity, one gets that $v$ is nondecreasing in both directions $Y$ and $-Y$. In other words, $v=v(X)$ and it satisfies the one-dimensional problem

$$
v^{\prime \prime}+c \sin \alpha v^{\prime}=0 \text { for } X>0, \quad v^{\prime}\left(0^{+}\right)=-c_{0} \text { and } v(+\infty)=0 .
$$

The function $u_{\infty}$ being globally Lipschitz-continuous in $\mathbb{R}^{2}$, the function $v$ is Lipschitz in $\mathbb{R}$ and in particular it is continuous at $X=0$. Therefore, $v(X)=\mathrm{e}^{-X c \sin \alpha}$ for $X \geqslant 0$ and the gradient condition at $X=0$ yields that $c_{0}=c \sin \alpha$.

Step 2: behaviour of $u$ along the lines $y=-|x| \cot \alpha$ as $x \rightarrow \pm \infty$. We here state and prove the following lemma, dealing with the behaviour of the functions

$$
u_{k}(x, y)=u\left(x+x_{k}, y-\left|x_{k}\right| \cot \alpha\right)
$$

where the sequence $\left(x_{k}\right)$ approaches either $-\infty$ ('left' side) or $+\infty$ ('right' side).

Lemma 5.3 The behaviour of the functions $u_{k}$ 'on the left side' is given by either

(a1) $\phi(x)+|x| \cot \alpha \rightarrow+\infty$ as $x \rightarrow-\infty$ and $u_{k} \rightarrow 0$ locally in $(x, y)$ for any sequence $x_{k} \rightarrow-\infty$, or

(a2) $\phi(x)+|x| \cot \alpha \rightarrow L^{-} \in \mathbb{R}$ as $x \rightarrow-\infty$ and, for any sequence $x_{k} \rightarrow-\infty, u_{k}(x, y) \rightarrow$ $U_{0}\left(-x \cos \alpha+y \sin \alpha-L^{-} \sin \alpha\right)$ locally in $(x, y)$, where $U_{0}(\xi)=\mathrm{e}^{c_{0} \xi}$ if $\xi \leqslant 0$ and $U_{0}(\xi)=1$ if $\xi \geqslant 0$.

The behaviour of the functions $u_{k}$ 'on the right side' is given by either

(b1) $\phi(x)+|x| \cot \alpha \rightarrow+\infty$ as $x \rightarrow+\infty$ and $u_{k} \rightarrow 0$ locally in $(x, y)$ for any sequence $x_{k} \rightarrow+\infty$, or

(b2) $\phi(x)+|x| \cot \alpha \rightarrow L^{+} \in \mathbb{R}$ as $x \rightarrow+\infty$ and, for any sequence $x_{k} \rightarrow+\infty, u_{k}(x, y) \rightarrow$ $U_{0}\left(x \cos \alpha+y \sin \alpha-L^{+} \sin \alpha\right)$ locally in $(x, y)$.

Proof. We only prove part'(a), part (b) being similar up to the change $x \rightarrow-x$. From Corollary 5.1 (ii), we know that the function $x \mapsto \phi(x)+|x| \cot \alpha$ is nonincreasing for $x \leqslant 0$. Therefore, one has as $x \rightarrow-\infty$ : either $\phi(x)+|x| \cot \alpha \rightarrow+\infty$ (a1), or $\phi(x)+|x| \cot \alpha \rightarrow L^{-} \in$ $\mathbb{R}(\mathrm{a} 2)$.

Let us first consider the case (a1). Choose any sequence $x_{k} \rightarrow-\infty$. Since $\phi$ is Lipschitzcontinuous and $\phi\left(x_{k}\right)+\left|x_{k}\right| \cot \alpha \rightarrow+\infty$, it follows that, for any compact subset $K \subset \mathbb{R}^{2}$, the set $E_{k}:=\left(x_{k},-\left|x_{k}\right| \cot \alpha\right)+K$ is included in $\Omega$ for $k$ large enough. Moreover, $d\left(E_{k}, \Gamma\right) \rightarrow+\infty$ as $k \rightarrow+\infty$. Therefore, the functions $u_{k}(x, y)$ approach 0 locally as $k \rightarrow+\infty$. 
Let us now turn to the second case (a2). As was done in step 1, one can prove that the (Lipschitz) functions $u_{k}(x, y)=u\left(x+x_{k}, y+\phi\left(x_{k}\right)\right)$ locally converge to the (Lipschitz) function $u_{\infty}(x, y)$ defined by: $u_{\infty}(x, y)=\mathrm{e}^{c_{0}(-x \cos \alpha+y \sin \alpha)}$ if $-x \cos \alpha+y \sin \alpha \leqslant 0$ and $u_{\infty}(x, y)=1$ otherwise. Using this fact and writing

$$
\begin{aligned}
u_{k}(x, y) & =u\left(x+x_{k}, y-\left|x_{k}\right| \cot \alpha\right) \\
& =u\left(x+x_{k}, y-\left(\phi\left(x_{k}\right)+\left|x_{k}\right| \cot \alpha\right)+\phi\left(x_{k}\right)\right)
\end{aligned}
$$

leads to the conclusion of Lemma 5.3 in case (a2).

As far as the function $u^{\alpha}$ given in Theorem 1.1 is concerned, it follows from Theorem 1.1 and Proposition 3.7 that $u^{\alpha}$ fulfills all the assumptions satisfied by the function $u$. Furthermore, the function $\phi^{\alpha}$ defined by $\left\{y=\phi^{\alpha}(x), x \in \mathbb{R}\right\}=\partial\left\{u^{\alpha}<1\right\}$ satisfies $\phi^{\alpha}(x)+|x| \cot \alpha \rightarrow L \in \mathbb{R}$ as $x \rightarrow \pm \infty$ (see Theorem 1.1). Hence, the previous lemma implies the following corollary.

Corollary 5.4 The functions $u^{\alpha}$ and $\phi^{\alpha}$ given in Theorem 1.1 satisfy the properties (a2) and (b2) of Lemma 5.3 with $L^{-}=L^{+}=L$.

Step 3: sliding $u^{\alpha}$ with respect to $u$ and end of the proof of Theorem 1.6. Let us now slide the function $u^{\alpha}$ given in Theorem 1.1 with respect to the function $u$, in the initial system of coordinates $(x, y)$.

Because of the formula (1.6) for $c$, and from Theorem 1.1 and Proposition 3.7, the functions $u$ and $u^{\alpha}$ satisfy

$$
\left\{\begin{aligned}
\Delta u-c \partial_{y} u=0 & \text { in } \Omega=\{u<1\}=\{y<\phi(x)\}, \\
\partial_{n} u=c_{0} & \text { on } \Gamma=\partial \Omega=\{y=\phi(x), x \in \mathbb{R}\}
\end{aligned}\right.
$$

and

$$
\left\{\begin{aligned}
\Delta u^{\alpha}-c \partial_{y} u^{\alpha}=0 & \text { in } \Omega^{\alpha}=\left\{u^{\alpha}<1\right\}=\left\{y<\phi^{\alpha}(x)\right\}, \\
\partial_{n} u^{\alpha}=c_{0} & \text { on } \Gamma^{\alpha}=\partial \Omega^{\alpha}=\left\{y=\phi^{\alpha}(x), x \in \mathbb{R}\right\}
\end{aligned}\right.
$$

in the sense of Definitions 3.1 and 3.3. For any $y_{0}$, let us set

$$
\left\{\begin{array}{l}
\Omega^{+}\left(y_{0}\right)=\left\{y>y_{0}+\phi^{\alpha}(x)\right\} \\
\Omega^{-}\left(y_{0}\right)=\left\{y<y_{0}+\phi^{\alpha}(x)\right\}
\end{array}\right.
$$

One has $u^{\alpha}=1$ in $\Omega^{+}(0)$. Lastly, since the graph $\Gamma^{\alpha}$ of the function $\phi^{\alpha}$ has asymptotes in the sense that

$$
\phi^{\alpha}(x)+|x| \cot \alpha \rightarrow L \text { as } x \rightarrow \pm \infty
$$

(see Theorem 1.1) and since the Lipschitz norm of $\phi^{\alpha}$ is less than or equal to $\cot \alpha$, one has $\phi^{\alpha}(x) \leqslant$ $-|x| \cot \alpha+L$ for all $x \in \mathbb{R}$. On the other hand, the Lipschitz norm of the function $\phi$ is not greater than $\cot \alpha$ either. Therefore, there exists a real number $h \in \mathbb{R}$ such that $\phi^{\alpha}(x) \leqslant \phi(x)+h$ for all $x \in \mathbb{R}$. That means that $\Omega^{-}\left(y_{0}\right) \subset\left\{y<y_{0}+\phi(x)+h\right\}$ for all $y_{0} \in \mathbb{R}$. Thus, one has

$$
\lim _{y_{0} \rightarrow-\infty} \sup _{\Omega^{-}\left(y_{0}\right)} u=0 .
$$

As a conclusion, the functions $u^{\alpha}$ and $u$ are respectively super- and subsolutions of a same free boundary problem of the type (3.2), in the sense of Section 4. Moreover, the same fact holds if one replaces the functions $u^{\alpha}$ or $u$ with $u^{\alpha}\left(x+h_{1}, y+h_{2}\right)$ and $u\left(x+h_{3}, y+h_{4}\right)$ for any $h_{1}, \ldots, h_{4} \in \mathbb{R}$. 
We shall now consider four cases (given by Lemma 5.3) and apply the comparison principle stated in Theorem 4.5. Recall here that $\Gamma=\partial\{u<1\}$ is equal to $\Gamma=\{y=\phi(x), x \in \mathbb{R}\}$.

First case: $\phi(x)+|x| \cot \alpha \rightarrow+\infty$ as $x \rightarrow \pm \infty$ (cases (a1) and (b1) of Lemma 5.3). We will see that this case is ruled out. Indeed, applying the comparison principle (Theorem 4.5) to $u^{\alpha}$ and $u$ leads to the existence of a (necessarily finite) real number $t^{*}$ such that $\left(u^{\alpha}\right)^{t^{*}}(x, y):=$ $u^{\alpha}\left(x, y+t^{*}\right) \geqslant u(x, y)$ in $\mathbb{R}^{2}$ and

(i) either $\left(u^{\alpha}\right)^{t^{*}}=u$ in $\mathbb{R}^{2}$,

(ii) or there exists a sequence of points $z_{k}=\left(x_{k}, y_{k}\right) \in\left(\Omega^{\alpha}\right)^{t^{*}}:=\Omega^{\alpha}-\left(0, t^{*}\right)$ such that

$$
\left|z_{k}\right| \rightarrow+\infty, \sup _{k} d\left(z_{k}, \Gamma^{\alpha}\right)<+\infty
$$

and both functions $v_{k}(x, y):=u^{\alpha}\left(x+x_{k}, y+y_{k}+t^{*}\right)$ and $u_{k}(x, y):=u\left(x+x_{k}, y+y_{k}\right)$ converge to the same limit $v_{\infty}=u_{\infty}$.

Case (i) is clearly impossible because $\left(\Gamma^{\alpha}\right)^{t^{*}}:=\partial\left\{\left(u^{\alpha}\right)^{t^{*}}<1\right\}$ has asymptotes parallel to the two half-lines $y=-|x| \cot \alpha$ as $x \rightarrow \pm \infty$, while $\Gamma=\partial\{u<1\}$ does not.

If case (ii) occurs, then, up to extraction of a subsequence, one has

$$
y_{k}+\left|x_{k}\right| \cot \alpha \rightarrow d \in \mathbb{R} \text { and } x_{k} \rightarrow \pm \infty .
$$

Hence, Lemma 5.3 (cases (a1) or (b1)) implies that $u_{\infty}=0$. On the other hand, Corollary 5.4 yields that $v_{\infty}$ is not zero. This case (ii) is then ruled out too.

Second case: $\phi(x)+|x| \cot \alpha \rightarrow L^{-}$as $x \rightarrow-\infty$ and $\phi(x)+|x| \cot \alpha \rightarrow+\infty$ as $x \rightarrow+\infty$ (cases (a2) and (b1) of Lemma 5.3). We shall prove here that the function $u$ is planar and that its level sets are parallel to the line $\{y=x \cot \alpha\}$. As in the first case, by applying Theorem 4.5, there exists a real number $t^{*}$ such that $\left(u^{\alpha}\right)^{t^{*}} \geqslant u$ in $\mathbb{R}^{2}$ and such that either (i) or (ii) occurs. Because of (b1), the behaviours of $\Gamma^{\alpha}$ and $\Gamma$ are asymptotically different as $x \rightarrow+\infty$ and we then see that case (i) cannot occur.

Consequently only case (ii) may occur. With the same notations and arguments as above, the case $x_{k} \rightarrow+\infty$ is ruled out. Hence, up to extraction of some subsequence, $x_{k} \rightarrow-\infty$. Furthermore, from Lemma 5.3 and Corollary 5.4, we can immediately check that

$$
v_{\infty}(x, y)=U_{0}\left(-x \cos \alpha+y \sin \alpha+\left(d+t^{*}-L\right) \sin \alpha\right),
$$

where $d=\lim y_{k}+\left|x_{k}\right| \cot \alpha$, and

$$
u_{\infty}(x, y)=U_{0}\left(-x \cos \alpha+y \sin \alpha+\left(d-L^{-}\right) \sin \alpha\right) .
$$

As a consequence, $t^{*}=L-L^{-}$.

Now take any real number $\eta \in \mathbb{R}$ and apply Theorem 4.5 to the functions

$$
u_{\eta}^{\alpha}(x, y)=u^{\alpha}(x+\eta, y+\eta \cot \alpha)
$$

and $u$ (the function $u_{\eta}^{\alpha}$ is obtained by translating $u^{\alpha}$ with a shift $-\eta / \sin \alpha$ in the direction $(\sin \alpha, \cos \alpha)$ ). There then exists a real number $\tau^{*}$ such that $\left(u_{\eta}^{\alpha}\right)^{\tau^{*}} \geqslant u$ and either (i) or (ii) occurs. As above, only case (ii) may occur and, owing to the choice of the function $u_{\eta}^{\alpha}$, it is found that $\tau^{*}=L-L^{-}=t^{*}$. Therefore, it follows that

$$
\forall \eta \in \mathbb{R}, \forall(x, y) \in \mathbb{R}^{2}, \quad u(x, y) \leqslant\left(u_{\eta}^{\alpha}\right)^{\tau^{*}}(x, y)=u^{\alpha}\left(x+\eta, y+\eta \cot \alpha+L-L^{-}\right) .
$$


By using Corollary 5.4 for the function $u^{\alpha}$, the limit $\eta \rightarrow-\infty$ yields

$$
\forall(x, y) \in \mathbb{R}^{2}, \quad u(x, y) \leqslant U_{0}\left(-x \cos \alpha+y \sin \alpha-L^{-} \sin \alpha\right) .
$$

On the other hand, since the function $u$ is nonincreasing in the direction $(-\sin \alpha,-\cos \alpha)$, one has

$$
u(x, y) \geqslant u(x+\lambda, y+|\lambda| \cot \alpha) \text { for all } \lambda \leqslant 0 \text { and }(x, y) \in \mathbb{R}^{2} .
$$

By using Lemma 5.3 for $u$, namely case (a2), the limit $\lambda_{k} \rightarrow-\infty$ in the previous inequality leads to

$$
\forall(x, y) \in \mathbb{R}^{2}, \quad u(x, y) \geqslant U_{0}\left(-x \cos \alpha+y \sin \alpha-L^{-} \sin \alpha\right) .
$$

Together with (5.4), that means that

$$
u(x, y) \equiv U_{0}\left(-x \cos \alpha+y \sin \alpha-L^{-} \sin \alpha\right) .
$$

In other words, the function $u$ is planar and its level sets are parallel to the line $\{y=x \cot \alpha\}$, which corresponds to the first case in the conclusion of Theorem 1.6.

Third case: $\phi(x)+|x| \cot \alpha \rightarrow+\infty$ as $x \rightarrow-\infty$ and $\phi(x)+|x| \cot \alpha \rightarrow L^{+}$as $x \rightarrow+\infty$ (cases (a1) and (b2) of Lemma 5.3). Like the second case, this case leads to

$$
u(x, y) \equiv U_{0}\left(x \cos \alpha+y \sin \alpha-L^{+} \sin \alpha\right)
$$

which corresponds to the second case in the conclusion of Theorem 1.6.

Fourth case: $\phi(x)+|x| \cot \alpha \rightarrow L^{ \pm} \in \mathbb{R}$ as $x \rightarrow \pm \infty$ (cases (a2) and (b2) of Lemma 5.3). We shall now prove that $u=u^{\alpha}$, up to translation. That will then complete the proof of Theorem 1.6.

Let us first define $h=\tan \alpha\left(L^{+}-L^{-}\right) / 2$ and $u_{h}(x, y)=u(x+h, y)$. From Lemma 5.3 applied to $u_{h}$, it then follows that

$$
u_{k}(x, y):=u_{h}\left(x+x_{k}, y-\left|x_{k}\right| \cot \alpha\right) \rightarrow U_{0}\left( \pm x \cos \alpha+y \sin \alpha-\frac{L^{+}+L^{-}}{2} \sin \alpha\right)
$$

for any sequence $x_{k} \rightarrow \pm \infty$. Roughly speaking, the function $u_{h}$ is then asymptotically symmetric in $x$ along the half-lines $\{y=-|x| \cot \alpha\}$ as $x \rightarrow \pm \infty$.

Let us now apply the comparison principle (Theorem 4.5) to the functions $u^{\alpha}$ (supersolution) and $u_{h}$ (subsolution). Under the same notation as above, there then exists a real number $t^{*}$ such that $\left(u^{\alpha}\right)^{t^{*}} \geqslant u_{h}$ in $\mathbb{R}^{2}$ and either case (i) or (ii) occurs, with $u$ being replaced with $u_{h}$.

If case (i) occurs, that corresponds to the third situation in the conclusion of Theorem 1.6.

Now assume that case (ii) occurs. Because of (5.5), one concludes as above that $t^{*}-L=$ $-\left(L^{+}+L^{-}\right) / 2$ (in each of the cases $x_{k} \rightarrow-\infty$ or $\left.x_{k} \rightarrow+\infty\right)$. On the other hand, because the conditions (a2) and (b2) are fulfilled, one has

$$
\sup _{x \in \mathbb{R}}|\phi(x)+| x|\cot \alpha|<\infty .
$$

One can then change the role of $u^{\alpha}$ and $u_{h}$ : namely, $u_{h}$ and $u^{\alpha}$ are respectively super- and subsolution of a problem of the type (3.2). Therefore, there exists a real number $\tau^{*}$ such that $u_{h}^{\tau^{*}} \geqslant u^{\alpha}$ in $\mathbb{R}^{2}$ and either case (i) or (ii) occurs (with $\tau^{*}, u_{h}, u^{\alpha}, \Omega$ in place of $t^{*}, u^{\alpha}, u$ and $\Omega^{\alpha}$ ). If case (i) 
occurs, then $u^{\alpha}$ and $u$ are equal up to translation and the conclusion of Theorem 1.6 is reached. Otherwise, case (ii) occurs and it is found as above that $\tau^{*}-\left(L^{+}+L^{-}\right) / 2=-L$, i.e. $\tau^{*}=-t^{*}$.

As a consequence, if $u^{\alpha}$ and $u$ were not equal up to translation, then one would have

$$
\left(u^{\alpha}\right)^{t^{*}} \geqslant u_{h} \text { and } u_{h}^{\tau^{*}} \geqslant u^{\alpha}
$$

with $t^{*}=-\tau^{*}$. The latter yields that

$$
u_{h}^{\tau^{*}} \equiv u^{\alpha} .
$$

As a conclusion, the functions $u^{\alpha}$ and $u$ are then equal up to translation.

This completes the proof of Theorem 1.6.

\section{Proof of the comparison principle (Theorem 4.5)}

This section is devoted to the proof of Theorem 4.5. The proof is based on the sliding method which has been developed by Berestycki and Nirenberg [10] and on some versions of the maximum principle in unbounded domains. Comparison principles similar to Theorem 4.5 had been obtained using the same device, in [7] and [27].

For the sake of clarity, the proof of Theorem 4.5 is divided into several steps. Let us start with the following version of the maximum principle.

Lemma 6.1 Let $\phi: \mathbb{R} \rightarrow \mathbb{R}$ be a globally Lipschitz-continuous function and let $D=\Omega^{-}\left(y_{0}\right)=$ $\left\{y<\phi(x)+y_{0}\right\}$, where $y_{0} \in \mathbb{R}$. Let $\bar{u}$ (resp. $\underline{u}$ ) be a Lipschitz-continuous function defined in $\bar{D}$ and such that $0 \leqslant \bar{u} \leqslant 1$ (resp. $0 \leqslant \underline{u} \leqslant 1)$ and

$$
L \bar{u} \leqslant 0 \text { in } \Omega(\bar{u})=\{X \in D, \bar{u}(X)<1\}
$$

resp.

$$
L \underline{u} \geqslant 0 \text { in } \Omega(\underline{u})=\{X \in D, \underline{u}(X)<1\},
$$

where

$$
L u=\Delta u+c_{1} \partial_{x} u+c_{2} \partial_{y} u .
$$

Assume that $\lim _{y \rightarrow-\infty} \sup _{\Omega^{-}(y)} \underline{u}=0$ and that

$$
\begin{cases}\underline{u} \leqslant \delta<1 & \text { in } \bar{D} \\ \underline{u} \leqslant \bar{u} & \text { on } \partial D\end{cases}
$$

Then $\underline{u} \leqslant \bar{u}$ in $\bar{D}$.

Proof. For all $\varepsilon>0$, set $\underline{u}_{\varepsilon}(x)=\underline{u}(x)-\varepsilon$ in $\bar{D}$. We shall prove that $\underline{u}_{\varepsilon} \leqslant \bar{u}$ in $\bar{D}$ for all $\varepsilon>0$. Since both $\bar{u}$ and $\underline{u}$ are bounded by 0 and 1 , one immediately has $\underline{u}_{1} \leqslant \bar{u}$ in $\bar{D}$. Define

$$
\varepsilon^{*}=\inf \left\{\varepsilon>0, \underline{u}_{\varepsilon} \leqslant \bar{u} \text { in } \bar{D}\right\} .
$$

Argue by contradiction and assume that $\varepsilon^{*}>0$. By continuity, one has $\underline{u}_{\varepsilon^{*}}=\underline{u}-\varepsilon^{*} \leqslant \bar{u}$ in $\bar{D}$. On the other hand, since $\lim _{y \rightarrow-\infty} \sup _{\Omega^{-}(y)} \underline{u}=0$, it follows that

$$
\underline{u}_{\varepsilon^{*}} \leqslant-\varepsilon^{*} / 2<0 \text { in } \overline{\Omega^{-}\left(y_{0}^{\prime}\right)}
$$


for some $y_{0}^{\prime}<y_{0}$. Since $\bar{u} \geqslant 0$, the minimality of $\varepsilon^{*}$ yields

$$
\sup _{\bar{D} \backslash \Omega^{-}\left(y_{0}^{\prime}\right)}\left(\underline{u}_{\varepsilon^{*}}-\bar{u}\right)=0
$$

Two cases can occur:

Case 1: there exists $X \in \overline{D \backslash \Omega^{-}\left(y_{0}^{\prime}\right)}$ such that

$$
\underline{u}_{\varepsilon^{*}}(X)\left(=\underline{u}(X)-\varepsilon^{*}\right)=\bar{u}(X) .
$$

In particular, $\bar{u}(X)<1$ and $X \in D$ since $\underline{u} \leqslant \bar{u}$ on $\partial D$. Since $\underline{u} \leqslant \delta<1$ in $D$, one has $L \underline{u} \geqslant 0$, whence $L \underline{u}_{\varepsilon^{*}} \geqslant 0$ in $D$. The nonpositive function $w=\underline{u}_{\varepsilon^{*}}-\bar{u}$ satisfies $L w \geqslant 0$ in $\Omega(\bar{u})$ and vanishes at the point $X$ which lies in the interior of this domain. From the strong maximum principle, it follows then that $\underline{u}_{\varepsilon^{*}} \equiv \bar{u}$ in the connected component of $\Omega(\bar{u})$ containing $X$. In this connected component, $\bar{u}=\underline{u}-\varepsilon^{*} \leqslant 1-\varepsilon^{*}<1$. As a consequence, this component is equal to the whole set $D$, whence

$$
\underline{u}_{\varepsilon^{*}} \equiv \bar{u} \text { in } \bar{D} .
$$

That is in contradiction with $\underline{u} \leqslant \bar{u}$ on $\partial D$.

Case 2: $\underline{u}_{\varepsilon^{*}}<\bar{u}$ in $\overline{D \backslash \Omega^{-}\left(y_{0}^{\prime}\right)}$. There exists then a sequence of points $\left(x_{k}, y_{k}\right) \in \overline{D \backslash \Omega^{-}\left(y_{0}^{\prime}\right)}$ such that

$$
\underline{u}_{\varepsilon^{*}}\left(x_{k}, y_{k}\right)-\bar{u}\left(x_{k}, y_{k}\right) \rightarrow 0 \text { as } k \rightarrow+\infty .
$$

On the other hand, the functions $\phi_{k}(x)=\phi\left(x_{k}+x\right)-\phi\left(x_{k}\right)$ converge locally uniformly, up to extraction of some subsequence, to a Lipschitz-continuous function $\phi_{\infty}$ and the functions

$$
\bar{u}_{k}(x, y)=\bar{u}\left(x_{k}+x, \phi\left(x_{k}\right)+y\right) \text { and } \underline{u}_{k}(x, y)=\underline{u}\left(x_{k}+x, \phi\left(x_{k}\right)+y\right)
$$

converge locally uniformly in $D_{\infty}=\left\{y<\phi_{\infty}+y_{0}\right\}$ to two functions $\bar{u}_{\infty}$ and $\underline{u}_{\infty}$. These functions are bounded between 0 and 1 (actually $\underline{u}_{\infty} \leqslant \delta<1$ in $D_{\infty}$ ) and they satisfy $L \bar{u}_{\infty} \leqslant 0$ (resp. $\left.L \underline{u}_{\infty} \geqslant 0\right)$ in $\mathcal{D}^{\prime}\left(D_{\infty} \cap \Omega\left(\bar{u}_{\infty}\right)\right)$ (resp. $\left.\mathcal{D}^{\prime}\left(D_{\infty}\right)\right)$. Furthermore, since $\bar{u}$ and $\underline{u}$ are globally Lipschitz, the functions $\bar{u}_{\infty}$ and $\underline{u}_{\infty}$ are also Lipschitz; they can then be extended by continuity on $\partial D_{\infty}$ and they satisfy $\underline{u}_{\infty} \leqslant \bar{u}_{\infty}$ on $\partial D_{\infty}$. Lastly, one has

$$
\lim _{y^{\prime} \rightarrow-\infty} \sup _{\left\{y<\phi_{\infty}(x)+y^{\prime}\right\}} \underline{u}_{\infty}(x, y)=0 .
$$

The passage to the limit $k \rightarrow+\infty$ leads to $\underline{u}_{\infty}-\varepsilon^{*} \leqslant \bar{u}_{\infty}$ whereas

$$
\underline{u}_{k}\left(0, y_{k}-\phi\left(x_{k}\right)\right)-\varepsilon^{*}-\bar{u}_{k}\left(0, y_{k}-\phi\left(x_{k}\right)\right) \rightarrow 0 .
$$

Since $\left(x_{k}, y_{k}\right) \in \bar{D} \backslash \Omega^{-}\left(y_{0}^{\prime}\right)$, one has $\phi\left(x_{k}\right)+y_{0}^{\prime} \leqslant y_{k} \leqslant \phi\left(x_{k}\right)+y_{0}$. Up to extraction of some subsequence, one can then assume that $y_{k}-\phi\left(x_{k}\right) \rightarrow y_{\infty} \in\left[y_{0}^{\prime}, y_{0}\right]$ as $k \rightarrow+\infty$. It then follows that $\underline{u}_{\infty}\left(0, y_{\infty}\right)-\varepsilon^{*}=\bar{u}_{\infty}\left(0, y_{\infty}\right)$ and one is led to a contradiction as in case 1 .

Both cases 1 and 2 are ruled out. The assumption $\varepsilon^{*}>0$ cannot hold and the proof of Lemma 6.1 is complete.

Let us now turn to the proof of Theorem 4.5.

Proof of Theorem 4.5. Under the assumptions of Theorem 4.5, there exists $y_{0} \in \mathbb{R}$ such that $\underline{u}<1 / 2$ in $D=\Omega^{-}\left(y_{0}\right)$. Recall that $\bar{u}=1$ in $\Omega^{+}\left(y_{1}\right)$. Therefore, for all $s \geqslant y_{1}-y_{0}$, one has $\bar{u}^{s}=1$ 
in $\Omega^{+}\left(y_{0}\right)$ and, in particular, $\bar{u}^{s} \geqslant \underline{u}$ on $\partial D$. The functions $\bar{u}^{s}$ and $\underline{u}$ meet the assumptions of Lemma 6.1 in $D$. As a consequence, $\overline{\bar{u}}^{s} \geqslant \underline{u}$ in $\bar{D}$, and then in $\mathbb{R}^{2}$ (since $\bar{u}^{s}=1$ in $\mathbb{R}^{2} \backslash \bar{D}$ ) for all $s \geqslant y_{1}-y_{0}$.

Therefore, the set $I=\left\{t \in \mathbb{R}, \bar{u}^{s} \geqslant \underline{u}\right.$ in $\mathbb{R}^{2}$ for all $\left.s \geqslant t\right\}$ is not empty. Assume from now on that $t^{*}=\inf I>-\infty$. By continuity, one has $\bar{u}^{t^{*}} \geqslant u$ in $\mathbb{R}^{2}$.

Two cases can occur:

Case $(i)$. There exists $X_{0} \in \Gamma\left(\bar{u}^{t^{*}}\right) \cap \Gamma(\underline{u})$. Since $\bar{u}^{t^{*}} \geqslant \underline{u}$ in $\mathbb{R}^{2}$, it follows that $\Gamma\left(\bar{u}^{t^{*}}\right)$ and $\Gamma(\underline{u})$ have the same normal at $X_{0}$. Since $\Gamma\left(\bar{u}^{t^{*}}\right)$ is regular in the sense of Definition 3.1, there exists an open ball $B$ of radius $\delta>0$ such that

$$
B \subset \Omega\left(\bar{u}^{t^{*}}\right) \text { and } X_{0} \in \partial B .
$$

In this ball, $\underline{u} \leqslant \bar{u}^{t^{*}}<1$, whence $B \subset \Omega(\underline{u})$ and $L z \leqslant 0$ in $B$ where $z=\bar{u}^{t^{*}}-\underline{u}$. Furthermore, owing to the choice of $X_{0}$, one has $z\left(X_{0}\right)=0$. The nonnegative function $z$ reaches its minimum 0 at the point $X_{0} \in \partial B$. On the other hand, since both $\bar{u}^{t^{*}}$ and $\underline{u}$ satisfy the same Neumann boundary condition in the sense of Definition 3.3 on $\Gamma\left(\bar{u}^{t^{*}}\right)$ and $\Gamma(\underline{u})$, it follows that $\partial_{n} z\left(X_{0}\right)=0$ where $n$ is the outward normal to $B$. The strong Hopf lemma yields $\overline{\bar{u}}^{t^{*}}=\underline{u}$ in the whole ball $B$.

Call $C^{-}$the connected component of $\Omega\left(\bar{u}^{t^{*}}\right)$ containing $B$. One has

$$
\bar{u}^{t^{*}}=\underline{u} \text { in } \overline{C^{-}} .
$$

In order to complete the proof of Theorem 4.5 in case (i), let us now prove that $C^{-}=\Omega\left(\bar{u}^{*}{ }^{*}\right)$.

Let us first observe that, if $(a, b) \in C^{-}$, then $(a, c) \in C^{-}$for all $c<b$. Otherwise, there would exist $c<b$ such that $(a, y) \in C^{-}$for all $y \in(c, b]$ and $(a, c) \in \partial C^{-}$. Therefore, $\bar{u}^{t^{*}}(a, c)=$ $\underline{u}(a, c)=1$ and $\bar{u}^{t^{*}}(a, y)=\underline{u}(a, y)<1$ for all $y \in(c, b]$. Hence,

$$
\bar{u}^{t^{*}+\varepsilon}(a, c)<1=\underline{u}(a, c) \text { for } \varepsilon>0 \text { small enough. }
$$

That contradicts the fact $\bar{u}^{t} \geqslant \underline{u}$ in $\mathbb{R}^{2}$ for all $t \geqslant t^{*}$ (from the definition of $t^{*}$ ).

Finally, $X_{1}+\mathbb{R}^{-} e_{y} \subset C^{-}$whenever $X_{1} \in C^{-}$. As a consequence, $C^{-} \cap \Omega^{-}\left(y_{0}\right)$ is not empty. Since $\underline{u}<1 / 2$ in $\Omega^{-}\left(y_{0}\right)$ whereas $\bar{u}^{*^{*}}=\underline{u}=1$ on $\partial C^{-}$, one concludes that $C^{-} \supset \Omega^{-}\left(y_{0}\right)$. Now assume that there exists a point $(x, y) \in \bar{\Omega}\left(\bar{u}^{t^{*}}\right) \backslash C^{-}$. One knows that $\left(x, y^{\prime}\right) \in C^{-}$for $-y^{\prime}$ large enough. Therefore, there exists a point $(x, \bar{y}) \in \partial C^{-}$such that $\bar{y}<y$. One has

$$
\bar{u}^{t^{*}}(x, \bar{y})=\underline{u}(x, \bar{y})=1 \text { and } \bar{u}^{t^{*}+y-\bar{y}}(x, \bar{y})=\bar{u}^{t^{*}}(x, y)<1=\underline{u}(x, \bar{y}) .
$$

Since $y>\bar{y}$, this contradicts the fact that $\bar{u}^{t} \geqslant u$ in $\mathbb{R}^{2}$ for all $t \geqslant t^{*}$.

To sum up, $C^{-}=\Omega\left(\bar{u}^{t^{*}}\right)$, whence $\bar{u}^{t^{*}}=\underline{u}$ in $\Omega\left(\bar{u}^{t^{*}}\right)$.

Case (ii). Assume that $\Gamma\left(\bar{u}^{t^{*}}\right) \cap \Gamma(\underline{u})=\emptyset$. In that case, one necessarily has $\bar{u}^{t^{*}}>\underline{u}$ in $\Omega\left(\bar{u}^{t^{*}}\right)$. Otherwise, there would exist a point $X_{1} \in \Omega\left(\bar{u}^{t^{*}}\right)$ such that $\bar{u}^{t^{*}}\left(X_{1}\right)=\underline{u}\left(X_{1}\right)$. The strong maximum principle would then imply that $\bar{u}^{t^{*}}=\underline{u}$ in the connected component $C^{-}$of $\Omega\left(\bar{u}^{t^{*}}\right)$ containing $X_{1}$. The set $C^{-}$is not the whole plane $\mathbb{R}^{2}$ because there are some points where $\bar{u}^{t^{*}}$ is equal to 1 . Therefore, $\partial C^{-}$is not empty. But any point $X_{0} \in \partial C^{-}$satisfies

$$
\bar{u}^{t^{*}}\left(X_{0}\right)=\underline{u}\left(X_{0}\right)=1,
$$

while $\bar{u}^{t^{*}}=\underline{u}<1$ in $C^{-}$. Finally, $\Gamma\left(\bar{u}^{t^{*}}\right) \cap \Gamma(\underline{u})$ contains the nonempty set $\partial C^{-}$. This is impossible. 
At this stage, one knows that $\bar{u}^{t^{*}}>\underline{u}$ in $\Omega\left(\bar{u}^{t^{*}}\right)$. Recall now that $\bar{u}^{t^{*}}=1$ in $\Omega^{+}\left(y_{1}-t^{*}\right)$ and $\underline{u}<1 / 2$ in $\Omega^{-}\left(y_{0}\right)$. Even if it means increasing $y_{1}$ or decreasing $y_{0}$, one can assume that the set

$$
E:=\left(\Omega^{-}\left(y_{1}+1-t^{*}\right) \backslash \Omega^{-}\left(y_{0}\right)\right) \cap \Omega\left(\bar{u}^{t^{*}}\right)
$$

is not empty.

In the case the nonnegative real number

$$
m=\inf _{E}\left(\bar{u}^{*}-\underline{u}\right)
$$

is positive, then there exists $\eta_{0}>0$ such that $\inf _{E}\left(\bar{u}^{*}-\eta-\underline{u}\right) \geqslant m / 2>0$ for all $\eta \in\left[0, \eta_{0}\right]$, since the function $\bar{u}$ is globally Lipschitz. Take any $\eta \in\left[0, \eta_{0}\right]$. It follows that

$$
\bar{u}^{*-\eta} \geqslant \min (1, \underline{u})=\underline{u} \text { in } \Omega^{-}\left(y_{1}+1-t^{*}\right) \backslash \Omega^{-}\left(y_{0}\right)
$$

and in particular on $\partial \Omega^{-}\left(y_{0}\right)$ while $\underline{u}<1 / 2$ in $\Omega^{-}\left(y_{0}\right)$. Lemma 6.1 yields that

$$
\bar{u}^{t^{*}-\eta} \geqslant \underline{u} \text { in } \overline{\Omega^{-}\left(y_{0}\right)} .
$$

On the other hand, $\bar{u}^{t^{*}}=1 \geqslant \underline{u}$ in $\Omega^{+}\left(y_{1}\right)$, whence $\bar{u}^{t^{*}-\eta}=1 \geqslant \underline{u}$ in $\Omega^{+}\left(y_{1}+1\right)$ as soon as $\eta \leqslant 1$. Finally, $\bar{u}^{t^{*}-\eta} \geqslant \underline{u}$ in $\mathbb{R}^{2}$ for all $0 \leqslant \eta \leqslant \min \left(\eta_{0}, 1\right)$. This contradicts the minimality of $t^{*}$.

Therefore,

$$
m=\inf _{E}\left(\bar{u}^{t^{*}}-\underline{u}\right)=0 .
$$

There then exists a sequence $\left(x_{k}, y_{k}\right) \in E$ such that $\bar{u}^{t^{*}}\left(x_{k}, y_{k}\right)-\underline{u}\left(x_{k}, y_{k}\right) \rightarrow 0$ as $k \rightarrow+\infty$.

Up to extraction of some subsequence, one can assume that $\left|x_{k}\right| \rightarrow+\infty$. Otherwise, $x_{k} \rightarrow x_{\infty}$ and $y_{k} \rightarrow y_{\infty}$ (the boundedness of $\left(x_{k}\right)$ implies the boundedness of $\left(y_{k}\right)$ ). Since $\left(x_{k}, y_{k}\right) \in \Omega\left(\bar{u}^{*}\right)$ and $\underline{u} \leqslant \bar{u}^{t^{*}}$, it would then follow that

$$
\underline{u}\left(x_{k}, y_{k}\right) \leqslant \bar{u}^{*}\left(x_{k}, y_{k}\right)<1 \text {. }
$$

By passage to the limit, one would get $\underline{u}\left(x_{\infty}, y_{\infty}\right)=\bar{u}^{t^{*}}\left(x_{\infty}, y_{\infty}\right)$ and either $\left(x_{\infty}, y_{\infty}\right) \in \Omega\left(\bar{u}^{t^{*}}\right)$ or $\left(x_{\infty}, y_{\infty}\right) \in \Gamma\left(\bar{u}^{t^{*}}\right) \cap \Gamma(\underline{u})$. One has proved that $\bar{u}^{t^{*}}>\underline{u}$ in $\Omega\left(\bar{u}^{t^{*}}\right)$, whence the first case is impossible. The second case is impossible by hypothesis. As a conclusion, $\left|x_{k}\right| \rightarrow+\infty$.

Let us now prove that both $d\left(\left(x_{k}, \phi\left(x_{k}\right)\right), \Gamma\left(\bar{u}^{t^{*}}\right)\right)$ and $d\left(\left(x_{k}, \phi\left(x_{k}\right)\right), \Gamma(\underline{u})\right)$ remain bounded. Since

$$
\left(x_{k}, y_{k}\right) \in E=\left(\Omega^{-}\left(y_{1}+1-t^{*}\right) \backslash \Omega^{-}\left(y_{0}\right)\right) \cap \Omega\left(\bar{u}^{t^{*}}\right)
$$

and $\bar{u}^{t^{*}}\left(x_{k}, \phi\left(x_{k}\right)+y_{1}-t^{*}\right)=1$, it follows that $d\left(\left(x_{k}, \phi\left(x_{k}\right)\right), \Gamma\left(\bar{u}^{t^{*}}\right)\right)$ as well as $d\left(\left(x_{k}, y_{k}\right), \Gamma\left(\bar{u}^{*}\right)\right)$ and $d\left(\left(x_{k}, y_{k}\right), \Gamma(\bar{u})\right)$ are bounded. In particular, Definition 3.3 then yields that $\liminf _{k \rightarrow+\infty} \bar{u}^{t^{*}}\left(x_{k}, y_{k}\right)>0$.

On the other hand, assume by contradiction that $d\left(\left(x_{k}, \phi\left(x_{k}\right)\right), \Gamma(\underline{u})\right) \rightarrow+\infty$. Since $\underline{u}<1 / 2$ in $\Omega^{-}\left(y_{0}\right)$ and $\left|y_{k}-\phi\left(x_{k}\right)\right|$ is bounded, it would then follow that $\left(x_{k}, y_{k}\right) \in \Omega(\underline{u})$ for $k$ large enough, whence $\underline{u}\left(x_{k}, y_{k}\right) \rightarrow 0$ because

$$
\limsup _{d(X, \Gamma(\underline{u})) \rightarrow+\infty, X \in \Omega(\underline{u})} \underline{u}=0 .
$$


Since $\liminf _{k \rightarrow+\infty} \bar{u}^{t^{*}}\left(x_{k}, y_{k}\right)>0$ and $\bar{u}^{t^{*}}\left(x_{k}, y_{k}\right)-\underline{u}\left(x_{k}, y_{k}\right) \rightarrow 0$, one has reached a contradiction. Finally, the sequence $d\left(\left(x_{k}, y_{k}\right), \Gamma(\underline{u})\right)$ is also bounded.

From Proposition 4.2, the functions $\phi_{k}(x)=\phi\left(x_{k}+x\right)-\phi\left(x_{k}\right)$ converge, up to extraction of some subsequence, to a Lipschitz function $\phi_{\infty}$, and the functions

$$
\bar{u}_{k}(x, y)=\bar{u}\left(x_{k}+x, \phi\left(x_{k}\right)+y\right) \text { and } \underline{u}_{k}(x, y)=\underline{u}\left(x_{k}+x, \phi\left(x_{k}\right)+y\right)
$$

converge to two functions $\bar{u}_{\infty}$ and $\underline{u}_{\infty}$, such that $\Gamma\left(\bar{u}_{\infty}\right)$ and $\Gamma\left(\underline{u}_{\infty}\right)$ are not empty, and solving (4.4) and (4.5). Furthermore,

$$
\bar{u}_{\infty}^{t} \geqslant \underline{u}_{\infty} \text { in } R^{2} \text { for all } t \geqslant t^{*} .
$$

Since $y_{k}-\phi\left(x_{k}\right)$ is bounded, one can also assume that $y_{k}-\phi\left(x_{k}\right) \rightarrow y_{\infty} \in \mathbb{R}$ as $k \rightarrow+\infty$. Therefore, $\bar{u}_{\infty}^{t^{*}}\left(X_{\infty}\right)=\underline{u}_{\infty}\left(X_{\infty}\right)$ where $X_{\infty}=\left(0, y_{\infty}\right)$. Lastly, since $\left(0, y_{k}-\phi\left(x_{k}\right)\right) \in \Omega\left(\bar{u}_{k}^{t^{*}}\right)$ and $\lim _{k \rightarrow+\infty} \Omega\left(\bar{u}_{k}^{t^{*}}\right)=\overline{\Omega\left(\bar{u}_{\infty}^{*}\right)}$ from Proposition 4.2, one gets that $X_{\infty} \in \overline{\Omega\left(\bar{u}_{\infty}^{t^{*}}\right)}$.

Two subcases may now occur:

Subcase (ii-a): $X_{\infty} \in \Gamma\left(\bar{u}_{\infty}^{*}\right)$. Then $\bar{u}_{\infty}^{t^{*}}\left(X_{\infty}\right)=1$, whence $\underline{u}_{\infty}\left(X_{\infty}\right)=1$ and $X_{\infty} \in \Gamma\left(\underline{u}_{\infty}\right) \cup$ $\left\{\underline{u}_{\infty}=1\right\}^{\mathrm{O}}$. If $X_{\infty} \in\left\{\underline{u}_{\infty}=1\right\}^{\mathrm{O}}$, then $1 \geqslant \bar{u}_{\infty}^{t^{*}} \geqslant \underline{u}_{\infty}=1$ in a neighbourhood of $X_{\infty}$, contradicting the fact that $X_{\infty} \in \Gamma\left(\bar{u}_{\infty}^{t^{*}}\right)$. Hence, $X_{\infty} \in \Gamma\left(\bar{u}_{\infty}^{t^{*}}\right) \cap \Gamma\left(\underline{u}_{\infty}\right)$. One then concludes as in case (i) above that

$$
\bar{u}_{\infty}^{t^{*}}=\underline{u}_{\infty} \text { in } \Omega\left(\bar{u}_{\infty}^{*}\right)
$$

Subcase (ii-b): $X_{\infty} \in \Omega\left(\bar{u}_{\infty}^{t^{*}}\right)$. Then the strong maximum principle implies that $\bar{u}_{\infty}^{t^{*}}=\underline{u}_{\infty}$ in the connected component $C_{\infty}^{-}$of $\Omega\left(\bar{u}_{\infty}^{t^{*}}\right)$ containing $X_{\infty}$. The boundary of $C_{\infty}^{-}$is not empty and it is then included in $\Gamma\left(\bar{u}_{\infty}^{t^{*}}\right) \cap \Gamma\left(\underline{u}_{\infty}\right)$. We then fall within case (ii-a) and (6.1) holds.

Therefore, the conclusion of Theorem 4.5 holds in case (ii).

Lastly, consider the case where both $\bar{u}$ and $\underline{u}$ are nondecreasing with respect to the variable $y$. Under the same notation as above, if case (i) occurs, then $\bar{u}^{*}$ and $u$ are nondecreasing in $y$ and since $\bar{u}^{t^{*}}$ and $u$ are equal in the set $\Omega\left(\bar{u}^{t^{*}}\right)$ which contains the set $\left\{y<\phi(x)+y_{0}\right\}$, it easily follows that $\bar{u}^{t^{*}}=\underline{u}$ in the whole plane $\mathbb{R}^{2}$. Similarly, if case (ii) occurs, then $\bar{u}_{\infty}^{t^{*}}=\underline{u}_{\infty}$ in $\mathbb{R}^{2}$. That completes the proof of Theorem 4.5 .

\section{Acknowledgements}

The authors are grateful to H. Berestycki, A. Bonnet, C.-M. Brauner and J. Busca for helpful comments and discussions during the preparation of this paper. The authors are also grateful to the referee for valuable comments. Part of this work was done by the second author at the Massachusetts Institute of Technology with the support of a NATO grant. The second author is indebted to these two institutions.

\section{REFERENCES}

1. Aftalion, A. \& Busca, J. Radial symmetry of overdetermined problems in exterior domains. Arch. Rat. Mech. Anal. 143, (1998) 195-206.

2. ANDREUCCI, D. \& GiANNI, R. Classical solutions to a multidimensional free boundary problem arising in combustion theory. Comm. Part. Diff. Eq. 19, (1994) 803-826. 
3. Aronson, D. G. \& Weinberger, H. F. Nonlinear diffusion in population genetics, combustion and nerve propagation, In: Part. Diff. Eq. and related topics. Lectures Notes in Math 446. Springer, New York (1975) pp. 5-49.

4. Berestycki, H., Caffarelli, L., \& Nirenberg, L. Uniform estimates for regularisation of free boundary problems. In: SADOSKY, C. \& DECKER, M. (eds), Anal. and Part. Diff. Eq.. (1990) pp. 567617.

5. Berestycki, H., Caffarelli, L., \& Nirenberg, L. Monotonicity for elliptic equations in unbounded Lipschitz domains. Comm. Pure Appl. Math. 50, (1997) 1089-1111.

6. Berestycki, H., CAfFarelli, L., \& Nirenberg, L. Singular limits in nonlinear elliptic equations and free boundary problems. Preprint (2002).

7. Berestycki, H., HAMEL, F., \& MonneaU, R. One-dimensional symmetry of bounded entire solutions of some elliptic equations. Duke Math. Journal 103, (2000) 375-396.

8. Berestycki, H. \& LARRouturou, B. In: BrÉZIS \& LIONS (eds), Quelques aspects mathématiques de la propagation des flammes prémélangées, Nonlinear p.d.e. and their applications, Collège de France seminar 10. Longman, Harlow (1990).

9. Berestycki, H., Nicolaenko, B., \& Scheurer, B. Traveling waves solutions to combustion models and their singular limits. SIAM J. Math. Anal. 16, (1985) 1207-1242.

10. Berestycki, H. \& Nirenberg, L. On the method of moving planes and the sliding method. Bol. da Soc. Braseleira de Matematica 22, (1991) 1-37.

11. Bonnet, A. \& HAMEL, F. Existence of a nonplanar solutions of a simple model of premixed Bunsen flames. SIAM J. Math. Anal. 31, (1999) 80-118.

12. Brauner, C.-M., Hulshof, J., \& Lunardi, A. A general approach to stability in free boundary problems. J. Diff. Equations 164, (2000) 16-48.

13. BRAUner, C.-M. \& LunARDi, A. Instabilities in a two-dimensional combustion model with free boundary. Arch. Ration. Mech. Anal. 154, (2000) 157-182.

14. Brauner, C.-M., Lunardi, A., \& Schmidt-Lainé, Cl. Stability of travelling waves with interface conditions. Nonlinear Analysis, Theo. Meth. Appl. 19, (1992) 455-474.

15. Buckmaster, J. D. A mathematical description of open and closed flame tips. Comb. Sci. Tech. 20, (1979) 33-40.

16. Buckmaster, J. D. \& Ludford, G. S. S. Theory of laminar flames, Electronic and Electrical Eng. Res: Pattern Recognition and Image Processing Series 2. Cambridge Univ. Press, New York (1982).

17. Buckmaster, J. D. \& Ludford, G. S. S. Lectures on Mathematical Combustion, CBMS-NSF Conf Series in Applied Math. 43, SIAM. (1983).

18. CAfFarelli, L. A. A Harnack inequality approach to the regularity of free boundaries, Part I: Lipschitz free boundaries are $C^{1, \alpha}$. Rev. Matem. Iberoamericana 3, (1987) 139-162.

19. Caffarelli, L. A., Lederman, C., \& Wolanski, N. Uniform estimates and limits for a two phase parabolic singular perturbation problem. Indiana Univ. Math. J. 46, (1997) 453-490.

20. Caffarelli, L. A. \& VAzQuez, J. L. A free-boundary problem for the heat equation arising in flame propagation. Trans. Am. Math. Soc. 347, (1995) 411-441.

21. FIfE, P. C. \& MCLEOD, J. B. The approach of solutions of nonlinear diffusion equations to traveling front solutions. Arch. Ration. Mech. Anal. 65, (1977) 335-361.

22. Frankel, M. L. \& Sivashinsky, G. I. On the equation of curved flame front. Physica D 30, (1988) $28-42$.

23. Galaktionov, V. A., Hulshof, J., \& VAzQuez, J. L. Extinction and focusing behaviour of spherical and annular flames described by a free boundary problem. J. Math. Pures Appl. 76, (1997) 563-608.

24. Gilbarg, D. \& Trudinger, N. S. Elliptic partial differential equations of second order. Springer, Berlin (1983).

25. Glangetas, L. Etude d'une limite singulière d'un modèle intervenant en combustion. Asym. Anal. 5, (1992) 317-342. 
26. Hamel, F. \& Monneau, R. Solutions d'équations elliptiques semilinéaires dans $\mathbb{R}^{N}$ ayant des courbes de niveau de forme conique. C. R. Acad. Sci. Paris 327 I, (1998) 645-650.

27. Hamel, F. \& Monneau, R. Solutions of semilinear elliptic equations in $\mathbb{R}^{N}$ with conical-shaped level sets. Comm. Partial Diff. Equations 25, (2000) 769-819.

28. Henrot, A. \& Philippin, G. A. On a class of overdetermined eigenvalue problems. Math. Methods Appl. Sci. 20, (1997) 905-914.

29. Hilhorst, D. \& Hulshof, J. A free boundary focusing problem. Proc. Ame. Math. Soc. 121, (1994) $1193-1202$.

30. Joulin, G. Dynamique des fronts de flammes, Modélisation de la combustion, Images des Mathématiques, CNRS. (1996).

31. KANEL', YA. I. Certain problems of burning-theory equations. Sov. Math. Dokl. 2, (1961) 48-51.

32. Kinderlehrer, D., Nirenberg, L., \& SPRUCK, J. Regularity in elliptic free boundary problems. I. J. Anal. Math. 34, (1978) 86-119.

33. Kozlovsky, G. \& Sivashinsky, G. I. On open and closed tips of Bunsen burner flames. Theor. Comput. Fluid Dyn 6, (1994) 181-192.

34. LEDERMAN, C. \& WOLANSKI, N. Viscosity solutions and regularity of the free boundary for the limit of an elliptic two phase singular perturbation problem. Ann. Scuola Norm. Sup. Pisa Cl. Sci. 27 (4), (1998) 253-288.

35. Lewis, B. \& Von Elbe, G. Combustion, Flames and Explosions of Gases. Academic, New York (1951).

36. LIÑAN, A. The structure of diffusion flames, Fluid dynamical aspects of combustion theory, Pitman Res. Notes Math. Ser. Longman Sci. Tech, Harlow 223, (1991) pp. 11-29.

37. Matkowsky, B. J. \& Sivashinsky, G. I. An asymptotic derivation of two models in flame theory associated with the constant density approximation. SIAM J. Appl. Math. 37, (1979) 686-699.

38. Michelson, D. Steady solutions of the Kuramoto-Sivashinsky equation. Phys. D 19, (1986) 89-111.

39. Michelson, D. Bunsen flames as steady solutions of the Kuramoto-Sivashinsky equation. SIAM J. Math. Anal. 23, (1991) 364-386.

40. Morrey, C. B. Multiple integrals in the calculus of variations. Springer, Berlin (1966).

41. NinOmiYA, H. \& TANiguCHI, M. Traveling curved fronts of a mean curvature flow with constant driving force. Free Boundary Problems, Theo. Applic., Mathe. Sci. Appli. 13, Gatuko Intern. Series. (2000) pp. 206-221.

42. NinOmiYA, H. \& TANiguchi, M. Stability of traveling curved fronts in a curvature flow with driving force. Meth. Appl. Analysis to appear.

43. REICHEL, W. Radial symmetry for elliptic boundary-value problems on exterior domains. Arch. Rat. Mech. Anal. 137, (1997) 381-394.

44. Serrin, J. A symmetry theorem in potential theory. Arch. Rat. Mech. Anal. 43, (1971) 304-318.

45. Sivashinsky, G. I. The diffusion stratification effect in Bunsen flames. J. Heat Transfer, Transactions of ASME 11, (1974) 530-535.

46. Sivashinsky, G. I. The structure of Bunsen flames. J. Chem. Phys. 62, (1975) 638-643.

47. SivAShinsky, G. I. Nonlinear analysis of hydrodynamics instability in laminar flames, I: Derivation of basic equations. Acta Astro. 4, (1977) 1177-1206.

48. Sivashinsky, G. I. On flame propagation under conditions of stoichiometry. SIAM J. Appl. Math. 39, (1980) 67-82.

49. Ton, B. A. On a free-boundary problem arising in the study of flame propagation. Math. Methods Appl. Sci. 15, (1992) 479-493.

50. Ton, B. A. On a steady state free boundary problem arising in combustion theory. Diff. Int. Eq. 5, (1992) 181-191.

51. Williams, F. Combustion Theory. Addison-Wesley, Reading, MA (1983).

52. Zeldovich, Y. B., Barenblatt, G. I., Libovich, V. B., \& Mackviladze, G. M. The mathematical theory of combustion and explosions. Cons. Bureau, New York (1985). 
53. Zeldovich, Y. B. \& Frank-Kamenetskit, D. A. A theory of thermal propagation of flame. In: PELCÉ, R. (ed), Dynamics of curved fronts, (Perspectives in Physics Series). Academic Press, New York (1988) pp. 131-140. 